University of Nebraska - Lincoln

DigitalCommons@University of Nebraska - Lincoln

Faculty Publications -- Chemistry Department Published Research - Department of Chemistry

$10-2010$

\title{
DNA-nanoparticle superlattices formed from anisotropic building blocks
}

\author{
Matthew R. Jones \\ Northwestern University \\ Robert J. Macfarlane \\ Northwestern University \\ Byeongdu Lee \\ Argonne National Laboratory \\ Jian Zhang \\ University of Nebraska-Lincoln, jzhang3@unl.edu \\ Kaylie L. Young \\ Northwestern University \\ See next page for additional authors
}

Follow this and additional works at: https://digitalcommons.unl.edu/chemfacpub

Part of the Chemistry Commons

Jones, Matthew R.; Macfarlane, Robert J.; Lee, Byeongdu; Zhang, Jian; Young, Kaylie L.; Senesi, Andrew J.; and Mirkin, Chad A., "DNA-nanoparticle superlattices formed from anisotropic building blocks" (2010). Faculty Publications -- Chemistry Department. 53.

https://digitalcommons.unl.edu/chemfacpub/53

This Article is brought to you for free and open access by the Published Research - Department of Chemistry at DigitalCommons@University of Nebraska - Lincoln. It has been accepted for inclusion in Faculty Publications -Chemistry Department by an authorized administrator of DigitalCommons@University of Nebraska - Lincoln. 


\section{Authors}

Matthew R. Jones, Robert J. Macfarlane, Byeongdu Lee, Jian Zhang, Kaylie L. Young, Andrew J. Senesi, and Chad A. Mirkin 


\title{
DNA-nanoparticle superlattices formed from anisotropic building blocks
}

\author{
Matthew R. Jones ${ }^{1,2}$, Robert J. Macfarlane ${ }^{2,3}$, Byeongdu Lee ${ }^{4}$, Jian Zhang ${ }^{2,3}$, Kaylie L. Young ${ }^{2,3}$, \\ Andrew J. Senesi ${ }^{2,3}$ and Chad A. Mirkin ${ }^{1,2,3 \star}$
}

\begin{abstract}
Directional bonding interactions in solid-state atomic lattices dictate the unique symmetries of atomic crystals, resulting in a diverse and complex assortment of three-dimensional structures that exhibit a wide variety of material properties. Methods to create analogous nanoparticle superlattices are beginning to be realized ${ }^{1-5}$, but the concept of anisotropy is still largely underdeveloped in most particle assembly schemes ${ }^{6}$. Some examples provide interesting methods to take advantage of anisotropic effects ${ }^{7-11}$, but most are able to make only small clusters or lattices that are limited in crystallinity and especially in lattice parameter programmability ${ }^{12-17}$. Anisotropic nanoparticles can be used to impart directional bonding interactions on the nanoscale ${ }^{6,18}$, both through face-selective functionalization of the particle with recognition elements to introduce the concept of valency ${ }^{19-21}$, and through anisotropic interactions resulting from particle shape ${ }^{13,22}$. In this work, we examine the concept of inherent shape-directed crystallization in the context of DNA-mediated nanoparticle assembly. Importantly, we show how the anisotropy of these particles can be used to synthesize one-, two- and threedimensional structures that cannot be made through the assembly of spherical particles.
\end{abstract}

Particle assembly is a rapidly developing field of research, because the properties of superlattices can be as different from their individual components as the physical properties of nanoparticles are from bulk materials ${ }^{6,14,18}$. Shape is an important parameter that affects the properties of a particle, and therefore the incorporation of anisotropic nanostructures into colloidal crystals should lead to materials with as yet undiscovered collective phenomena ${ }^{6}$. Maximum utility of these structures requires that a technique be developed to rationally integrate particles with non-spherical shapes into ordered assemblies. The use of DNA as a ligand for the three-dimensional (3D) crystallization of nanoparticles into colloidal superlattices has many advantages over other assembly techniques ${ }^{1-4}$. In particular, the synthetically programmable length and recognition properties of DNA have enabled researchers to generate well-defined superlattices from spherical nanoparticles that vary in size (5-80 $\mathrm{nm}$; ref. 23). Moreover, the technique provides nanometre-scale precision in programming the resulting lattice parameters. By replacing the spherical cores that associate through isotropic hybridization interactions with anisotropic nanostructures, we hypothesized that directional bonding interactions could be facilitated by virtue of different particle shapes (Fig. 1a).

Dense DNA functionalization of anisotropic nanostructures (previously demonstrated only for a single anisotropic nanostructure ${ }^{21}$ ) was achieved through a combination of particle purification, overgrowth or appropriate surfactant mixtures. These procedures allow one to prepare monodisperse $(<10 \%)$, pure ( $>95 \%$ ) solutions of DNA-functionalized triangular nanoprisms ${ }^{24}$, nanorods ${ }^{25}$, rhombic dodecahedra ${ }^{26}$ and octahedra ${ }^{26}$ (see Supplementary Information for details of the synthesis and functionalization procedures, Fig. 1b). Nanoparticle assembly occurred through the hybridization of DNA linkers (containing 23, 64, 105, 146 and 187 nucleobases) to the oligonucleotides anchored to the particles (Fig. 1d). On binding to the nanoparticle, these linkers present multiple 'sticky ends' at a programmable distance from the nanoparticle surface, creating, in essence, a controllably sized 'DNA shell' that directs the crystallization process ${ }^{23}$. Cooperative melting transitions (where 'melting' refers to the dehybridization of DNA bases linking particles) have been measured for crystals formed in solution for each of these particle shapes, indicating a dense surface coverage of oligonucleotides ${ }^{27}$, which is essential for the assembly and subsequent crystallization process ${ }^{23}$ (Fig. 1c).

Small-angle X-ray scattering (SAXS) was used to interrogate the colloidal crystal structures synthesized from anisotropic particles. SAXS represents a powerful characterization tool for this new class of solution-phase-assembled nanostructures, and can give the symmetries, lattice constants, particle orientation and domain size of a $3 \mathrm{D}$ ordered structure without requiring drying of the sample (see Table 1), which significantly affects the resulting lattices ${ }^{23}$. Modelling of nanoparticle superlattices has been carried out to corroborate these results by comparing modelled SAXS patterns to those obtained experimentally. Additionally, transmission electron micrographs of several ordered nanoparticle crystals, embedded in a resin, support the conclusions drawn from the SAXS data and have been included in the Supplementary Information.

The first systems examined were assemblies created from primarily $1 \mathrm{D}$ structures (nanorods), where a '1D' particle is defined as having a length significantly greater than its width or depth. As has been previously shown with spherical particles, the most stable crystal structure for a given system is typically the one with the largest number of DNA linker interactions ${ }^{3}$; one would therefore predict that these rods would preferentially assemble with their long axes parallel to each other, to maximize the hybridization events between DNA sticky ends. It is important to note that there is a difference between the processes of particle 'crystallization' and particle 'assembly'. In this context, particle crystallization refers to positioning particles in an ordered formation that has translational symmetry, whereas assembly refers to the process of DNA-hybridizationinduced association, regardless of the structure of the aggregate. It has previously been demonstrated that the process of particle assembly occurs on a significantly faster timescale than the process of

\footnotetext{
${ }^{1}$ Department of Materials Science and Engineering, Evanston, Illinois 60208-3113, USA, ${ }^{2}$ International Institute for Nanotechnology, Northwestern University, 2145 Sheridan Road, Evanston, Illinois 60208-3113, USA, ${ }^{3}$ Department of Chemistry, Evanston, Illinois 60208-3113, USA, ${ }^{4}$ Advanced Photon Source, Argonne National Laboratory, Argonne, Illinois 60439, USA. *e-mail: chadnano@northwestern.edu.
} 
a
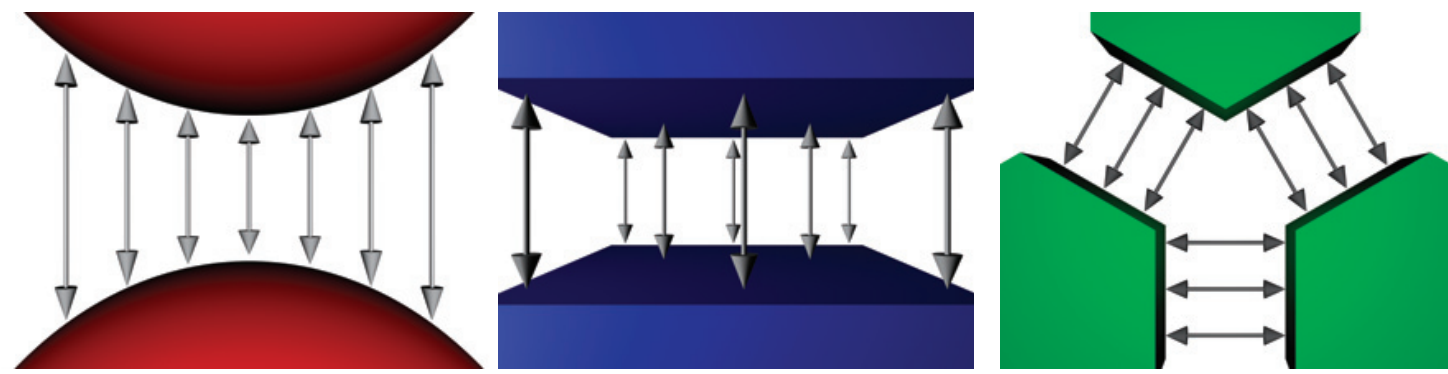

b
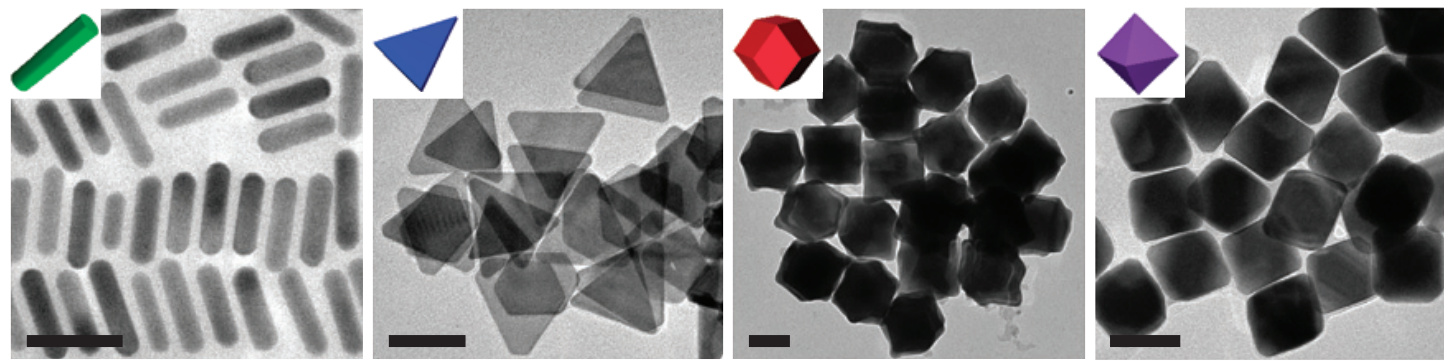

C
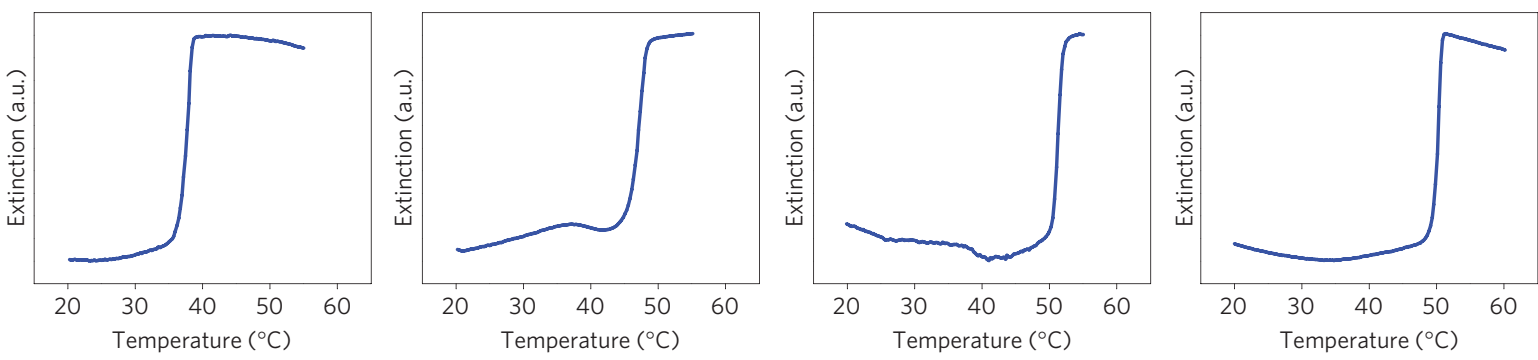

d

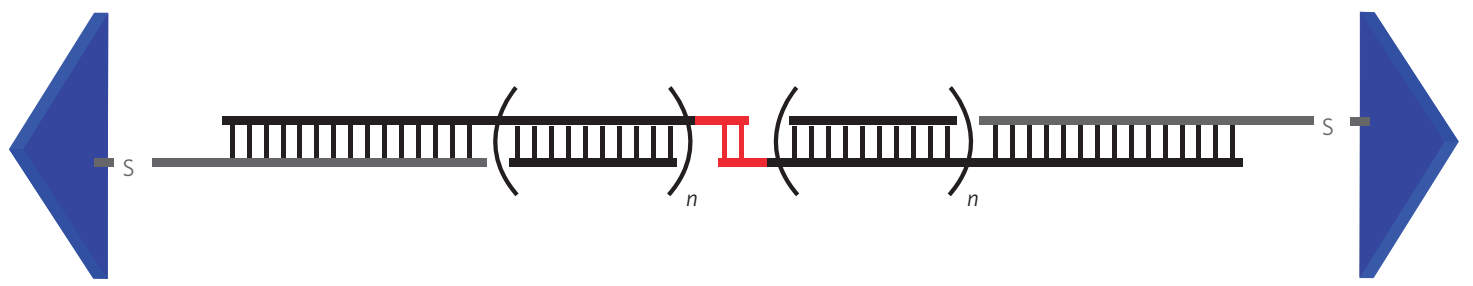

Figure 1 | Directional bonding interactions can be imparted to monodisperse, DNA-functionalized gold nanostructures through the introduction of shape anisotropy. a, The curved surfaces of spherical particles (left) cannot support the same number of idealized oligonucleotide interactions without DNA deformation as the flat, faceted surfaces of anisotropic nanostructures (middle). This feature allows for the shape of a nanostructure to more strongly dictate the structural details of the assembled superlattice it composes (right). b. Transmission electron microscopy images of (from left to right) rods, triangular prisms, rhombic dodecahedra and octahedra. The scale bars represent $50 \mathrm{~nm}$. c. Extinction monitored at surface plasmon resonance maximum (from left to right: rods $-800 \mathrm{~nm}$, prisms $-1,200 \mathrm{~nm}$, rhombic dodecahedra- $618 \mathrm{~nm}$, octahedra $-550 \mathrm{~nm}$ ) as a function of temperature for DNA-functionalized anisotropic nanostructures assembled with linker oligonucleotides. The sharp melting transitions are indicative of a dense surface coverage of DNA. $\mathbf{d}$, Schematic of the oligonucleotides used to assemble anisotropic nanostructures. Thiolated DNA strands (grey) anchored to the particle's surface were hybridized to linker DNA (black), which contained modular blocks of a repeated 40-base-pair sequence (labelled ' $n$ ', where $n$ refers to the number of 40-base segments) and a short self-complementary (GCGC) recognition sequence (red) that induced particle assembly.

particle crystallization ${ }^{28}$. Therefore, in all cases presented here, one can visually observe the initial formation of large, disordered aggregates and differentiate the subsequent ordering events using SAXS.

Gold nanorods were assembled using DNA linkers of varying lengths; on annealing, the resulting superlattices possessed longrange hexagonal symmetry (Fig. 2a,b, Table 1). Most crystals exhibited scattering peaks corresponding only to $2 \mathrm{D}$ ordering (Supplementary Figs S5, S6, S21). However, extended thermal annealing of some samples resulted in ordering between $2 \mathrm{D}$ sheets, with the resulting peaks indexing to a $P 6_{3} / m m c$, hexagonal-closepacked, lattice. As nanorods were observed to order into $2 \mathrm{D}$ sheets in most samples studied, one can conclude that the particles favour interactions perpendicular to their long axis, demonstrating that the ' $1 D$ ' shape is directing their crystallization into primarily a
2D lattice, and these $2 \mathrm{D}$ lattices can subsequently reorganize into an ordered 3D structure. This was further probed through in situ monitoring of the crystallization process (Supplementary Fig. S22), wherein peaks corresponding to $2 \mathrm{D}$ order appear and begin to sharpen (indicating growth in crystal domain size) significantly before any peaks corresponding to 3D order appear.

The assembly of triangular nanoprisms was then investigated as an example of a ' $2 D$ ' nanostructure, wherein the length and width of a prism are an order of magnitude greater than its depth. One would expect the most dominant crystallization force to be face-to-face interactions between the predominantly 2D nanoprisms. Scattering patterns from superstructures of assembled prisms indicate a lamellar (or columnar) 1D arrangement of particles stacked in a face-to-face configuration (Fig. 2c,d). This is consistent with the 
Table 1 | Summary of crystallization parameters for DNA-functionalized anisotropic nanoparticle superlattices.

\begin{tabular}{lllll} 
& $\begin{array}{l}\text { Nanoparticle } \\
\text { dimensions (nm) }\end{array}$ & $\begin{array}{l}\text { Lattice } \\
\text { parameters (nm) }\end{array}$ & $\begin{array}{l}\text { Average crystal } \\
\text { domain sizes (nm) }\end{array}$ & $\begin{array}{l}\text { Average no. unit } \\
\text { cells/crystal }\end{array}$ \\
\hline Rods & $14(\mathrm{w}) \times 55(\mathrm{l})$ & $34.3,55.8,77.9,97.9,110$ & $1,270 \pm 310$ & $330 \pm 210$ \\
& $19(\mathrm{w}) \times 52(\mathrm{l})$ & $34.3,57.7,77.6,93.9,113$ & $1,180 \pm 650$ & $220 \pm 120$ \\
Triangular & $60(\mathrm{e}) \times 7$ (w) & $26.2,48.3,71.8,94.3,113$ & $750 \pm 230$ & $14 \pm 4$ \\
prisms & $95(\mathrm{e}) \times 7$ (w) & $23.6,48.6,72.1,94.9,114$ & $980 \pm 400$ & $18 \pm 6$ \\
& $140(\mathrm{e}) \times 7(\mathrm{w})$ & $24.4,48.9,72.3,93.3,114$ & $1,040 \pm 510$ & $19 \pm 6$ \\
Rhombic & $39(\mathrm{e}), 64(\mathrm{~d})$ & 181,201 & $2,550 \pm 990$ & $1,160 \pm 810$ \\
dodecahedra & $50(\mathrm{e}), 81(\mathrm{~d})$ & 198,220 & $1,780 \pm 490$ & $420 \pm 120$ \\
Octahedra & $59(\mathrm{e}), 83(\mathrm{~d})$ & $110,118(\mathrm{bcc}) ; 135,159$ (fcc) & $1,510 \pm 170$ & $910 \pm 470$
\end{tabular}

Nanoparticle dimensions were obtained from transmission electron microscopy images and lattice parameters, domain size and unit cells per crystal were obtained from SAXS data ( $w$ - width, I-length, e-edge length, $d$-diameter). Calculations can be found in the Supplementary Information.

a

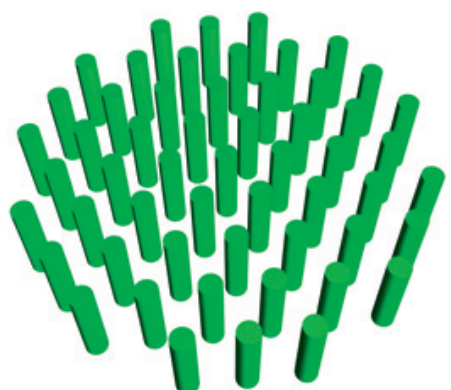

b

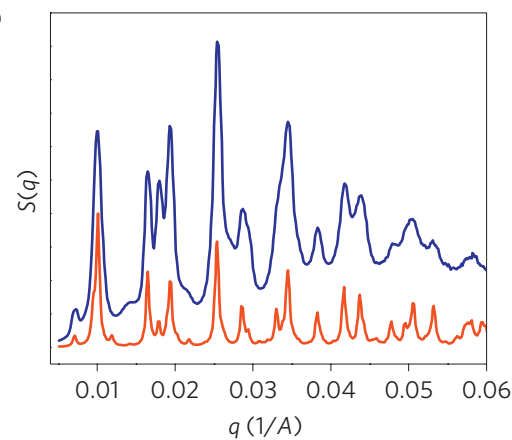

c

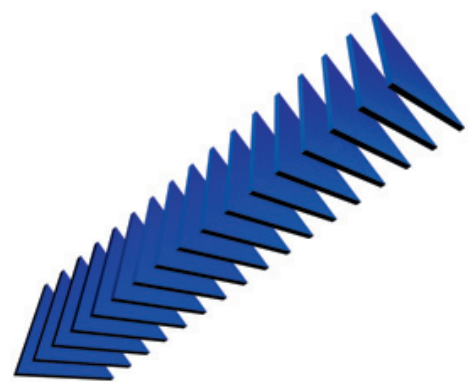

d

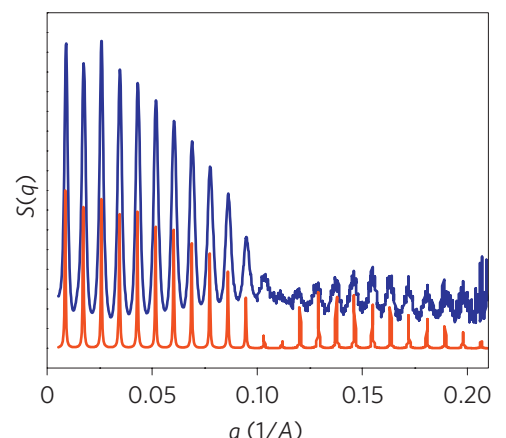

e

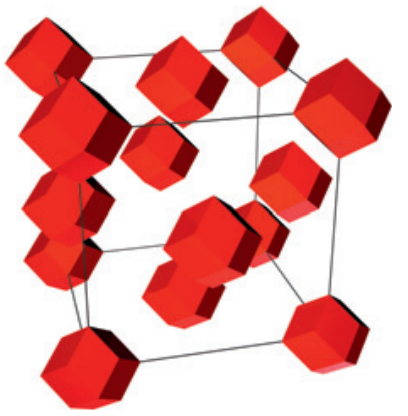

f

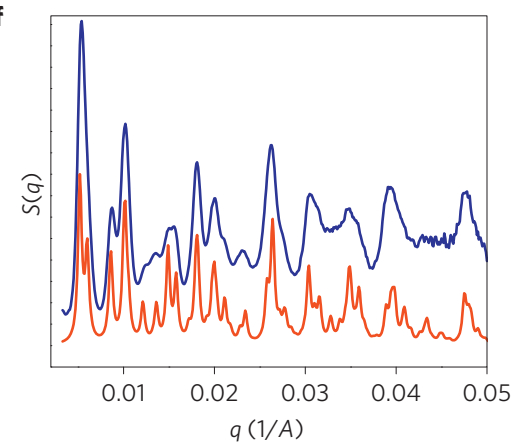

Figure 2 | SAXS characterization of anisotropic nanoparticle colloidal crystals. a, Schematic of a hexagonal 2D layer (additional layers omitted for clarity) in assemblies of gold nanorods. b, Experimental (blue) and simulated (red) SAXS patterns for gold nanorods ( $55 \mathrm{~nm}$ length, $14 \mathrm{~nm}$ diameter) assembled into a hexagonal-close-packed lattice with lattice constants of $a=76.0 \mathrm{~nm}$ and $c=176.5 \mathrm{~nm}$. c, Schematic of the primarily $1 \mathrm{D}$ lamellar assemblies of gold triangular nanoprisms. d, Experimental (blue) and simulated (red) SAXS patterns for nanoprisms ( $95 \mathrm{~nm}$ edge length, $7 \mathrm{~nm}$ thickness) assembled into a columnar arrangement with a lattice spacing of $72.1 \mathrm{~nm}$. e, Schematic of the 3D fcc assemblies of gold rhombic dodecahedra. The lines denote the fcc unit cell, not interparticle interactions. f, Experimental (blue) and simulated (red) SAXS patterns for rhombic dodecahedra (64 nm diameter) assembled into an $\mathrm{fcc}$ arrangement with a lattice constant of $201.4 \mathrm{~nm}$.

previous observation (see above) that particles associate in a manner that maximizes hybridization interactions. Unlike the primarily 2D hexagonally packed nanorods, no long-range DNA-mediated ordering was observed between 1D stacks of prisms. Some crystals that underwent significant thermal annealing exhibited short-range order between nanoprism superlattices (Supplementary Fig. S23), but these scattering patterns could not be attributed to a welldefined 3D lattice. This lack of 3D ordering can be explained by the inherent thinness of the prisms $(7 \mathrm{~nm})$ and the relative rigidity of double-stranded DNA (persistence length of $\sim 50 \mathrm{~nm}$; ref. 29), resulting in very low DNA density along the side of a column. As the long axis of a $1 \mathrm{D}$ stack of prisms contains a relatively diffuse coating of DNA sticky ends, we project that hierarchical crystallization of these columns is not favourable enough to produce 3D structures in the timescales monitored herein (see Supplementary Information for more detail).

A high degree of precision over the placement of prisms in $1 D$ chains can be seen qualitatively by the large number of diffraction peaks present in the scattering pattern. Quantification of the rise per base pair (particle face-to-face distance divided by number of DNA bases) for three different particle sizes (edge lengths $=60,95,140 \mathrm{~nm}$ ) and three different linker lengths (DNA lengths $=40.5,63.5,86.5 \mathrm{~nm}$ ) yields a value of $0.281 \pm 0.002 \mathrm{~nm}$. This is a remarkable level of precision and programmability over the placement of nanomaterials along one dimension that would be difficult, if not impossible, to replicate by any lithographic or other directed-assembly method, illustrating one of the primary advantages of the DNA-directed crystallization methodology. 


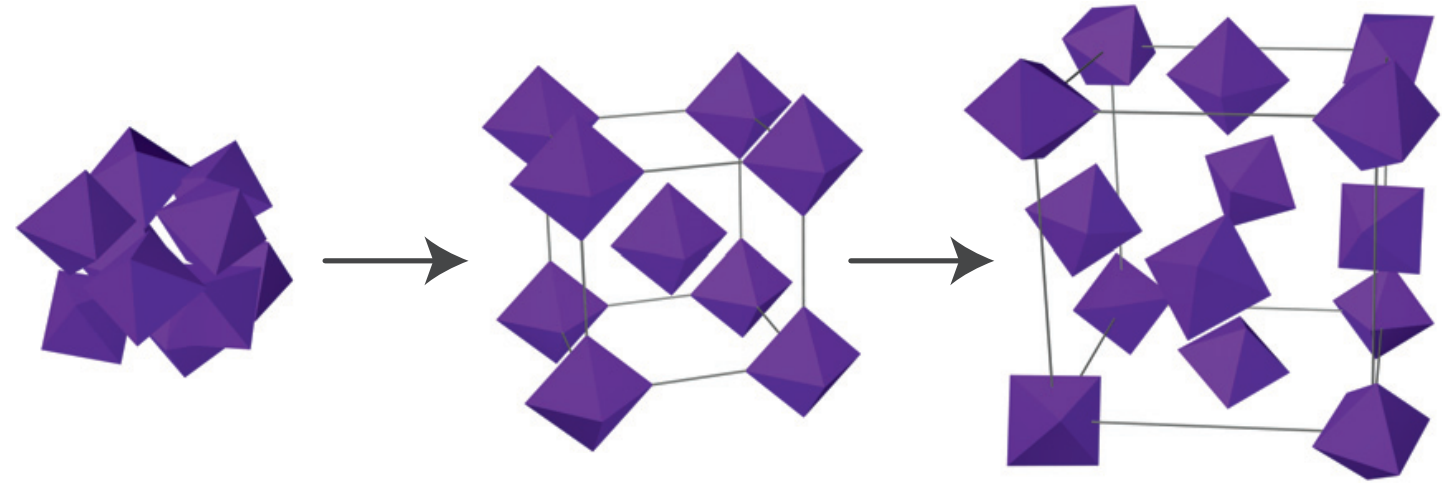

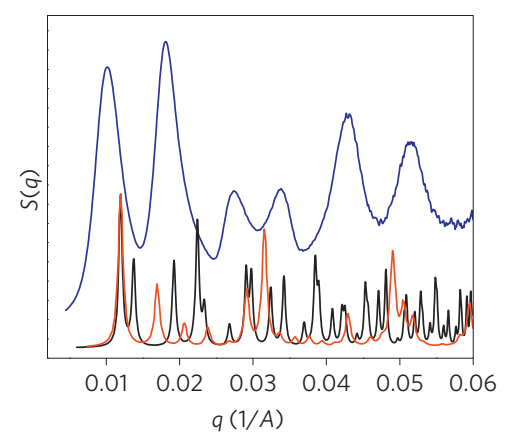

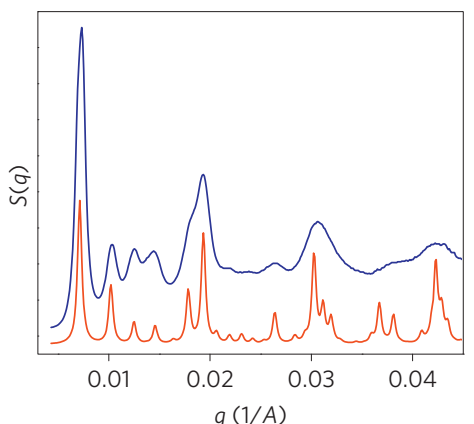

d

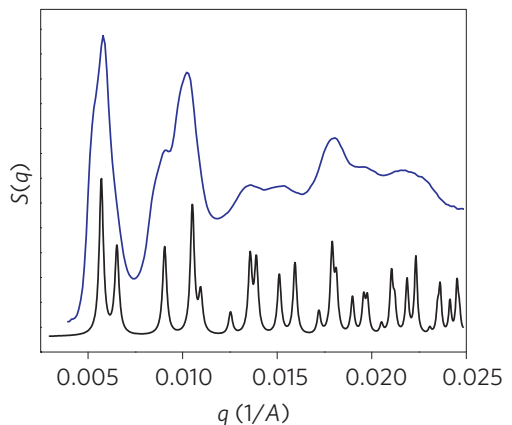

Figure 3 | Nanoparticle colloidal crystals undergo phase transformations as a function of DNA length. a, Schematic of disordered, bcc and fcc phases of crystallized octahedral nanoparticles, which are stable at short, intermediate and long DNA lengths, respectively. b, Experimental (blue) SAXS characterization of gold octahedra ( $59 \mathrm{~nm}$ edge length) assembled with short DNA ( $15 \mathrm{~nm}$ ) resulting in an interparticle distance of $76.8 \mathrm{~nm}$. Simulated SAXS patterns for bcc (red) and fcc (grey) crystals with lattice parameters that would be expected given the length of the linking DNA strands demonstrate that the particles do not correlate with either lattice. Calculations and further explanation can be found in the Supplementary Information. c, Experimental (blue) and simulated (red) SAXS patterns for octahedra assembled with DNA linkers of an intermediate length ( $45 \mathrm{~nm}$ ) resulting in face-to-face orientational ordering within a bcc crystal with a lattice parameter of $118.4 \mathrm{~nm}$. d. Experimental (blue) and simulated (grey) SAXS patterns for octahedra assembled with long DNA $(\sim 90 \mathrm{~nm})$ resulting in no orientational ordering within an fcc crystal with a lattice parameter of $194.7 \mathrm{~nm}$.

In accordance with the previous definitions of particle dimensionality (see above), a '3D' object would have no physical dimension significantly larger or smaller than the other two. The rhombic dodecahedron is an ideal particle to investigate the role of shape in 3D nanoparticle crystallization, as it naturally forms a face-centred-cubic (fcc) lattice with $100 \%$ packing efficiency, contrasted with previous fcc lattices of spherical particles ${ }^{3,23}$, which exhibit a packing density of only $\sim 74 \%$. Unlike spheres, which interact in an isotropic fashion along a curved surface, maximum DNA interactions for the rhombic dodecahedron system would be obtained only when the particles associate face-to-face and are ordered in an fcc lattice with both positional and orientational order. Comparing the crystallization of rhombic dodecahedra to similarly sized spheres would give a good indication of how shape anisotropy affects positional and rotational order and crystal quality in the resulting colloidal superlattices.

The SAXS data confirm that the rhombic dodecahedra crystallize into fcc lattices and retain the lattice parameter programmability imparted by variations in DNA linker length (Fig. 2e,f, Supplementary Figs S10 and S11). Importantly, the lattices exhibit a significantly larger number of scattering peaks than fcc crystals consisting of similarly sized spherical particles ${ }^{23}$, indicating greater positional order with respect to an ideal fcc lattice. Relative intensities of diffraction peaks correlate with modelled SAXS patterns for lattices wherein the rhombic dodecahedra retain orientational order (Supplementary Information, Figs S24-S29). This ordering occurs even when the hydrodynamic size of the 'DNA shell' is significantly larger than that of the particle, confirming that the rhombic dodecahedron shape does indeed have a strong influence on colloidal crystal formation. From these data, one can conclude that the incorporation of shape anisotropy provides significant benefit to packing precision over the assembly of isotropic spheres.

It is important to note that the DNA linkers directing the crystallization process are not completely rigid. With increasing DNA length, one would expect the increasing flexibility of the DNA strands to change the sphericity of the DNA shell. Rhombic dodecahedra crystallized in fcc lattices retain their orientational order, indicating that the shape of their DNA shell is similar over all DNA lengths examined. Octahedra are another interesting class of particle worth probing in this manner. Experimentally and theoretically, octahedra pack most densely in lattices that do not maximize commensurate face-to-face interactions ${ }^{30-32}$. Therefore, octahedral nanoparticles allow one to test the hypothesis that DNAdriven particle assembly and crystallization favours the structure that maximizes hybridization events and, in the case of anisotropic nanostructures, face-to-face interactions.

SAXS data demonstrate that the octahedra crystallize into a disordered lattice, a body-centred-cubic (bcc) lattice and an fcc lattice, with short, intermediate and long DNA lengths, respectively (Fig. 3a). With short DNA, only short-range order is observed-the corresponding scattering pattern does not index with either a bcc or fcc lattice, the two structures observed for longer DNA lengths (Fig. 3b). Although several possible lattices have been proposed for the dense packing of octahedra without DNA (refs 30-32), with short, inflexible DNA, none of these would produce a stable structure, according to the hypothesis, because they all exhibit severely limited face-to-face overlap (see Supplementary Information). At a DNA length of $\sim 45 \mathrm{~nm}$, however, the scattering peaks index to a bcc lattice; the intensities of these peaks are best fitted by a model that includes face-to-face orientational ordering between 
octahedra (Fig. 3c). As the DNA is more flexible, the shape of the DNA shell may appear more like a truncated octahedron, which is able to maximize face-to-face interactions in a bcc arrangement (see Supplementary Information). At a DNA length of $\sim 90 \mathrm{~nm}$, the scattering pattern indexes to an fcc lattice, indicative of a particle that is more similar to a sphere than a well-defined octahedron (Fig. 3d). In this case, the intensities of the peaks indicate no orientational alignment of particles, as would be expected of a DNA shell that is approximately spherical. These data indicate that, at all DNA lengths, shape has a significant impact on the most stable structure, but, depending on DNA flexibility, the particle and the DNA have differing levels of importance in determining the anisotropy of the interactions between DNA-functionalized particles. Therefore, the interplay between the two components of this bionanoconjugate crystallization methodology provides a means to control the phase behaviour of a colloidal superlattice constructed from a given set of anisotropic nanostructure building blocks.

We have demonstrated that particle shape has a strong influence on the crystallization parameters of DNA-functionalized nanoparticles, affecting superlattice dimensionality, crystallographic symmetry and phase behaviour. Furthermore, the use of DNA as a programmable linker imparts the ability to tune the lattice parameters of the resulting crystals while retaining the shape directing effects of the nanoparticles within the limits of DNA flexibility. Moreover, this work is consistent with the conclusion that nanoparticle superlattices that can maximize interparticle DNA hybridization events will be the most stable structures. Such crystals may find use in applications that take advantage of the ability to tune the unique physical properties of these structures, such as plasmonic-based circuitry or waveguides, photonic bandgap materials and energy harvesting or storage materials, all of which exhibit unique emergent properties that are dependent on interparticle distance and crystal symmetry. In particular, the precision with which we can position particles is difficult to replicate using other assembly or lithographic techniques, indicating that this methodology provides a powerful means to realize these types of designer materials. Furthermore, we project that these results will enable fundamental insights into shape-directed hybridization effects and the influence of nanostructure valency on crystallographic parameters.

\section{Received 6 May 2010; accepted 31 August 2010; published online 3 October 2010}

\section{References}

1. Mirkin, C. A., Letsinger, R. L., Mucic, R. C. \& Storhoff, J. J. A DNA-based method for rationally assembling nanoparticles into macroscopic materials. Nature 382, 607-609 (1996).

2. Park, S-J., Lazarides, A. A., Storhoff, J. J., Pesce, L. \& Mirkin, C. A. The structural characterization of oligonucleotide-modified gold nanoparticle networks formed by DNA hybridization. J. Phys. Chem. B 108, 12375-12380 (2004).

3. Park, S. Y. et al. DNA-programmable nanoparticle crystallization. Nature 451, 553-556 (2008)

4. Nykypanchuk, D., Maye, M. M., van der Lelie, D. \& Gang, O. DNA-guided crystallization of colloidal nanoparticles. Nature 451, 549-552 (2008).

5. Shevchenko, E. V., Talapin, D. V., Kotov, N. A., O’Brien, S. \& Murray, C. B. Structural diversity in binary nanoparticle superlattices. Nature 439, 55-59 (2006).

6. Glotzer, S. C. \& Solomon, M. J. Anisotropy of building blocks and their assembly into complex structures. Nature Mater. 6, 557-562 (2007).

7. Lapointe, C. P., Mason, T. G. \& Smalyukh, I. I. Shape-controlled colloidal interactions in nematic liquid crystals. Science 326, 1083-1086 (2009).

8. Sacanna, S., Irvine, W. T. M., Chaikin, P. M. \& Pine, D. J. Lock and key colloids. Nature 464, 575-578 (2010).

9. Zerrouki, D., Baudry, J., Pine, D., Chaikin, P. \& Bibette, J. Chiral colloidal clusters. Nature 455, 380-382 (2008).

10. Srivastava, S. et al. Light-controlled self-assembly of semiconductor nanoparticles into twisted ribbons. Science 327, 1355-1359 (2010).

11. DeVries, G. A. et al. Divalent metal nanoparticles. Science 315, 358-361 (2007).

12. Liu, Q. et al. Self-alignment of plasmonic gold nanorods in reconfigurable anisotropic fluids for tunable bulk metamaterial applications. Nano Lett. 10, 1347-1353 (2010).
13. Ming, T. et al. Ordered gold nanostructure assemblies formed by droplet evaporation. Angew. Chem. Int. Edn 120, 9831-9836 (2008).

14. Tao, A., Sinsermsuksakul, P. \& Yang, P. Tunable plasmonic lattices of silver nanocrystals. Nature Nanotechnol. 2, 435-440 (2007).

15. Alivisatos, A. P. et al. Organization of 'nanocrystal molecules' using DNA. Nature 382, 609-611 (1996).

16. Wei, Y., Bishop, Kyle J. M., Kim, J., Soh, S. \& Grzybowski, B. A. Making use of bond strength and steric hindrance in nanoscale 'Synthesis'. Angew. Chem. Int. Edn 121, 9641-9644 (2009).

17. Li, M., Schnablegger, H. \& Mann, S. Coupled synthesis and self-assembly of nanoparticles to give structures with controlled organization. Nature 402, 393-395 (1999).

18. Stebe, K. J., Lewandowski, E. \& Ghosh, M. Oriented assembly of metamaterials. Science 325, 159-160 (2009).

19. Nie, Z. et al. Self-assembly of metal-polymer analogues of amphiphilic triblock copolymers. Nature Mater. 6, 609-614 (2007).

20. Li, F., Yoo, W. C., Beernink, M. B. \& Stein, A. Site-specific functionalization of anisotropic nanoparticles: From colloidal atoms to colloidal molecules. J. Am. Chem. Soc. 131, 18548-18555 (2009).

21. Millstone, J. E. et al. DNA-gold triangular nanoprism conjugates. Small 4, 2176-2180 (2008).

22. Zhang, Z. \& Glotzer, S. C. Self-assembly of patchy particles. Nano Lett. 4, 1407-1413 (2004).

23. Macfarlane, R. J. et al. Establishing the design rules for DNA-mediated programmable colloidal crystallization. Angew. Chem. Int. Edn 49, 4589-4592 (2010)

24. Millstone, J. E. et al. Observation of a quadrupole plasmon mode for a colloidal solution of gold nanoprisms. J. Am. Chem. Soc. 127, 5312-5313 (2005).

25. Nikoobakht, B. \& El-Sayed, M. A. Preparation and growth mechanism of gold nanorods (NRs) using seed-mediated growth method. Chem. Mater. 15, 1957-1962 (2003).

26. Niu, W. et al. Selective synthesis of single-crystalline rhombic dodecahedral, octahedral, and cubic gold nanocrystals. J. Am. Chem. Soc. 131, 697-703 (2008).

27. Jin, R., Wu, G., Li, Z., Mirkin, C. A. \& Schatz, G. C. What controls the melting properties of DNA-linked gold nanoparticle assemblies? J. Am. Chem. Soc. 125, 1643-1654 (2003).

28. Macfarlane, R. J. et al. Assembly and organization processes in DNA-directed colloidal crystallization. Proc. Natl Acad. Sci. USA 106, 10493-10498 (2009).

29. Rivetti, C., Walker, C. \& Bustamante, C. Polymer chain statistics and conformational analysis of DNA molecules with bends or sections of different flexibility. J. Mol. Biol. 280, 41-59 (1998).

30. Lu, W. et al. Super crystal structures of octahedral c- $\operatorname{In}_{2} \mathrm{O}_{3}$ nanocrystals. J. Am. Chem. Soc. 130, 6983-6991 (2008).

31. Xie, S. et al. Supercrystals from crystallization of octahedral MnO nanocrystals. J. Phys. Chem. C 113, 19107-19111 (2009).

32. Torquato, S. \& Jiao, Y. Dense packings of the Platonic and Archimedean solids. Nature 460, 876-879 (2009).

\section{Acknowledgements}

C.A.M. acknowledges the NSF-NSEC and the AFOSR for grant support, and the DOE Office (Award No. DE-SC0000989) for support through the NU Nonequilibrium Energy Research Center. He is also grateful for an NSSEF Fellowship from the DoD. M.R.J. acknowledges Northwestern University for a Ryan Fellowship and the NSF for a Graduate Research Fellowship. R.J.M. acknowledges Northwestern University for a Ryan Fellowship. K.L.Y. acknowledges the NSF and the NDSEG for Graduate Research Fellowships. Portions of this work were carried out at the DuPont-Northwestern-Dow Collaborative Access Team (DND-CAT) beamline located at Sector 5 of the Advanced Photon Source (APS). DND-CAT is supported by E.I. DuPont de Nemours \& Co., The Dow Chemical Company and the State of Illinois. Use of the APS was supported by US Department of Energy, Office of Science, Office of Basic Energy Sciences, under Contract No. DE-AC02-06CH11357. The transmission electron microscope work was carried out in the EPIC facility of NUANCE Center at Northwestern University. NUANCE Center is supported by NSF-NSEC, NSF-MRSEC, Keck Foundation, the State of Illinois and Northwestern University. Ultrathin sectioning was carried out at the Northwestern University Biological Imaging Facility supported by the NU Office for Research.

\section{Author contributions}

M.R.J. and R.J.M. designed the systems, prepared the materials, collected and analysed the data and wrote the manuscript. B.L. analysed the data, carried out simulations of nanoparticle superlattices and wrote the manuscript. J.Z., K.L.Y. and A.J.S. collected data. C.A.M. designed the systems and wrote the manuscript.

\section{Additional information}

The authors declare no competing financial interests. Supplementary information accompanies this paper on www.nature.com/naturematerials. Reprints and permissions information is available online at http://npg.nature.com/reprintsandpermissions. Correspondence and requests for materials should be addressed to C.A.M. 


\section{Supplementary Information}

\section{DNA-Nanoparticle Superlattices Formed From Anisotropic Building Blocks}

Matthew R. Jones, Robert J. Macfarlane, Byeongdu Lee, Jian Zhang, Kaylie L. Young, Andrew J. Senesi, and Chad A. Mirkin*

[*] Prof. C. A. Mirkin

Department of Chemistry

Department of Materials Science and Engineering

International Institute for Nanotechnology

Northwestern University, 2145 Sheridan Road, Evanston, Illinois 60208-3113, USA

Fax: (+1) 847-467-5123

Email: Chad A. Mirkin (chadnano@northwestern.edu) 


\section{Materials and Methods}

\section{Anisotropic Nanostructure DNA Functionalization}

Although each particle functionalization presented unique individual challenges, all procedures are generally based on a previously demonstrated ligand replacement reaction between cetyltrimethylammonium bromide (CTAB) and thiolated oligonucleotides ${ }^{1}$. Briefly, CTAB $(0.05 \mathrm{M})$ stabilized anisotropic particles were spun down twice and resuspended in nanopure (18.2 M $\Omega-\mathrm{cm}$ ) water. Thiolated oligonucleotides (see Table S1 for sequences) that had been oxidized with $0.1 \mathrm{M}$ dithiothreitol (DTT) and purified with a desalting column were then immediately added to the colloidal solution ( $3 \mathrm{OD}_{260}$ of DNA per $\mathrm{mL}$ of nanoparticle colloid). Surface reorganization effects or irreversible particle aggregation were only observed if more than 20-30 minutes were allowed between the final resuspension in water and the addition of thiolated DNA. After allowing 1-3 hours for thiolated DNAs to react with the gold surface, particle suspensions were brought to $0.01 \%$ sodium dodecyl sulfate (SDS) and $0.01 \mathrm{M}$ sodium phosphate and allowed to sit for 30 min to 1 hour. Following literature precedent ${ }^{2}$, the colloidal particle solutions were then slowly treated with $\mathrm{NaCl}$ to allow for electrostatic screening between neighboring DNA strands and denser surface coverage of oligonucleotides. Specifically, solutions were brought to $0.05 \mathrm{M}, 0.1 \mathrm{M}, 0.2 \mathrm{M}, 0.3 \mathrm{M}, 0.4 \mathrm{M}$, and $0.5 \mathrm{M} \mathrm{NaCl}$ sequentially with approximately $30 \mathrm{~min}$ between each salt addition. After reaching the final salt concentration, particles were allowed to sit overnight to achieve maximum DNA loading. In order to remove unreacted oligonucleotides from solution, particle suspensions were centrifuged, the supernatant was removed, and the pellet was resuspended in $0.01 \%$ SDS three times. The final resuspension typically occurred in $50-100 u$ to allow for a concentrated solution of particles. Sodium phosphate and $\mathrm{NaCl}$ were added to bring the final suspension to $0.01 \mathrm{M}$ and $0.5 \mathrm{M}$ sodium phosphate and $\mathrm{NaCl}$, respectively. For all cases studied, as long as anisotropic particles could be stably prepared or transferred to $0.05 \mathrm{M} \mathrm{CTAB}$, oligonucleotide functionalization following the above procedure resulted in a dense surface coverage of oligonucleotides and well ordered assemblies mediated through the addition of DNA linkers (see below for details of the synthesis and functionalization of each nanostructure). 


\section{Gold Nanorod Synthesis, Overgrowth, and Functionalization}

Gold nanorods were synthesized using the silver-assisted growth procedure developed by El-Sayed and coworkers ${ }^{3}$. We observed that nanorods made in this way could not be functionalized with oligonucleotides as evidenced by either particle aggregation during the salting step or a lack of cooperative melting behavior, which indicates low DNA density on a nanoparticle's surface ${ }^{4}$. We hypothesized that the difficulty in conjugating thiolated DNA to the particle was due to a thin layer of silver present on the nanorod surface preventing Au-S bond formation. This feature has been proposed as part of the mechanism for the silver-assisted nanorod growth ${ }^{5}$. In order to overcome this, an additional overgrowth procedure was used to deposit a thin layer of gold on the nanorod, which allowed for facile DNA functionalization.

As-synthesized nanorods were spun down (10 min, $13400 \mathrm{rcf}$ ) once and resuspended in water, followed by a second centrifugation to concentrate the particles with resuspension in 0.01 M CTAB. Nanorod concentrations were quantified using optical extinction spectra and the absorbance value at the longitudinal surface plasmon resonance (LSPR) maximum. An extinction coefficient of $1.1 \times 10^{9} \mathrm{M}^{-1} \mathrm{~cm}^{-1}$ was used for rods with aspect ratio $\sim 4$ and an extinction coefficient of $9.1 \times 10^{8} \mathrm{M}^{-1} \mathrm{~cm}^{-1}$ was used for rods with aspect ratio $\sim 3$. A $900 \mathrm{pM}$ solution of nanorods was then prepared in $0.01 \mathrm{M} \mathrm{CTAB}$ and sequentially brought to $1 \mathrm{mM}$ ascorbic acid and $0.005 \mathrm{mM} \mathrm{HAuCl}_{4}$. After allowing $30 \mathrm{~min}$ to 1 hour for the overgrowth to occur, the solution was then brought to $0.05 \mathrm{M} \mathrm{CTAB}$ and functionalized with DNA according to the previously outlined procedure (vide supra). The overgrown nanorods showed minimal change in their optical properties and their dimensions (Figure S1) but showed a significant improvement in their ability to be functionalized with oligonucleotides (Figure S2).

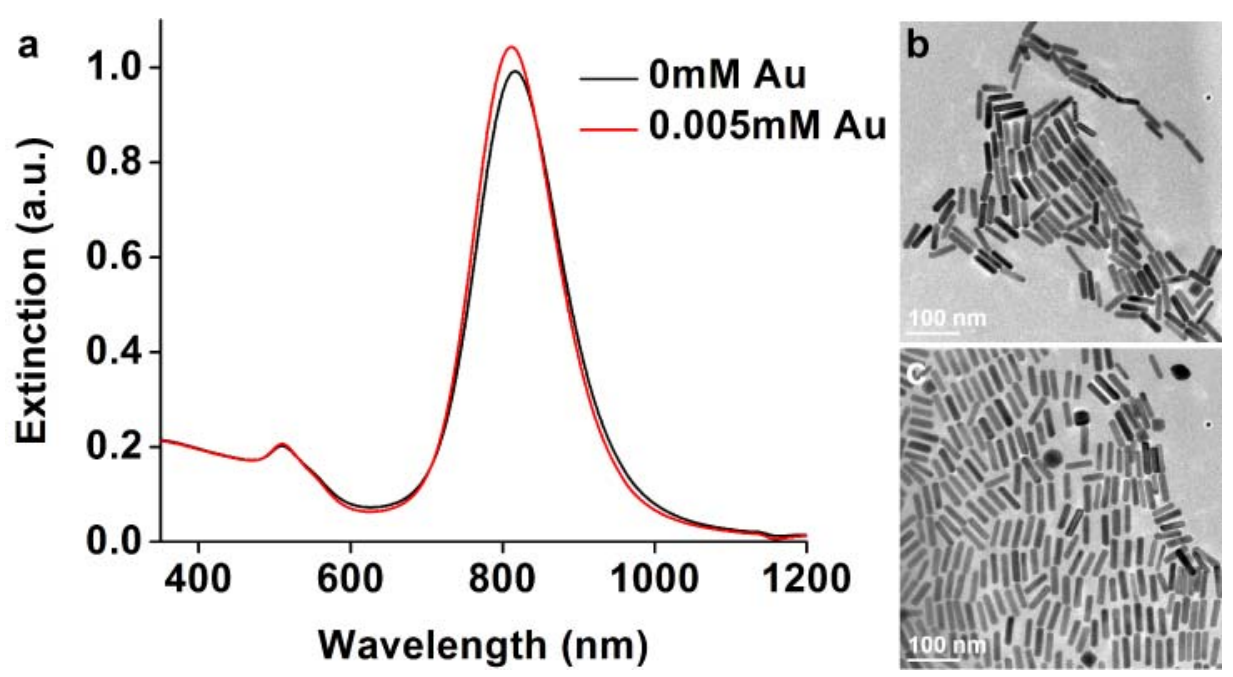

Figure S1. Optimization of nanorod overgrowth. a, Extinction spectra recorded from gold nanorods before (black) and after (red) overgrowth procedure. b-c, TEM images before (b) and after (c) overgrowth procedure. Nanorod dimensions in $\mathbf{b}$ are 54 $\mathrm{nm} \pm 10 \%$ length, $14 \mathrm{~nm} \pm 12 \%$ width. Nanorod dimensions in c are $55 \mathrm{~nm} \pm 7 \%$ length, $14 \mathrm{~nm} \pm 10 \%$ width. 

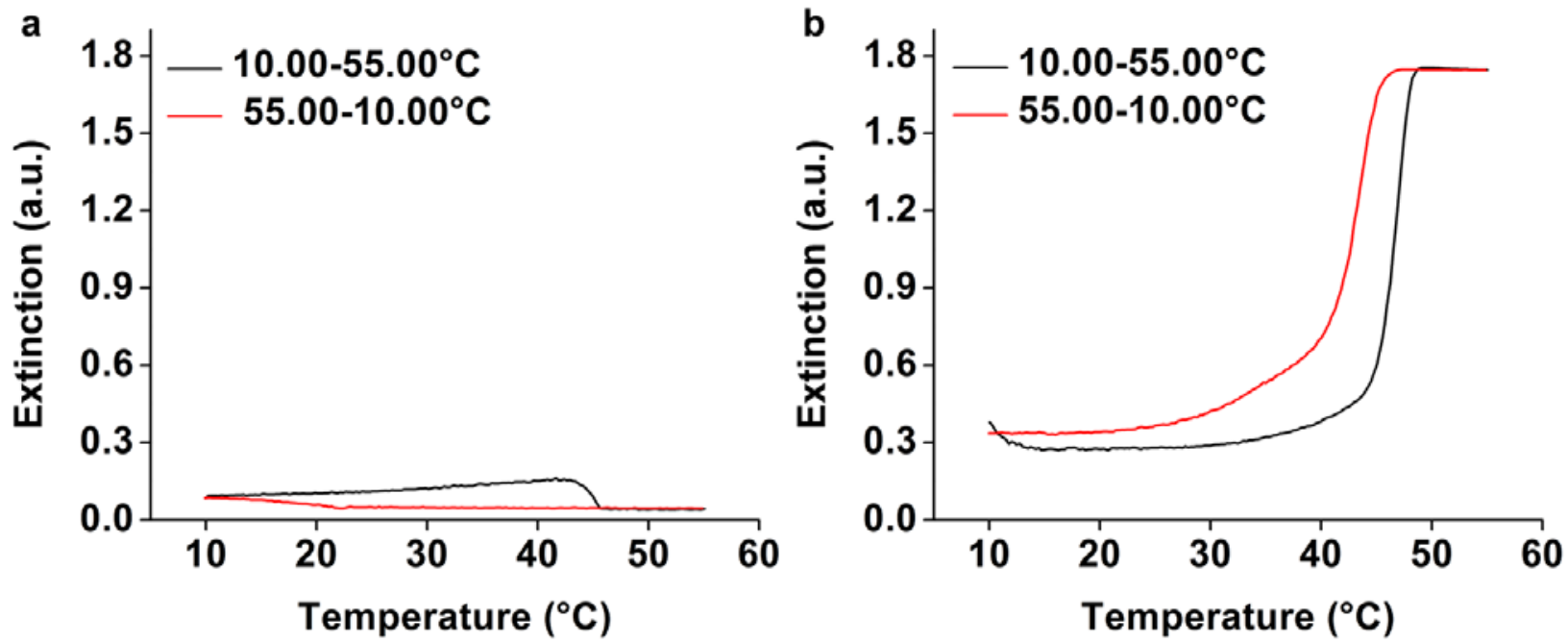

Figure S2. Confirmation of DNA functionalization of gold nanorods. a-b, Extinction monitored at particle surface plasmon resonance $(800 \mathrm{~nm})$ as a function of temperature for nanorods treated with thiolated DNA and assembled with linker oligonucleotides according to the included procedure. a, As-synthesized gold nanorods. b, Nanorods that had been treated with the overgrowth procedure. The sharp melting transition in $\mathbf{b}$ (and lack of a similar transition in a) indicates dense surface coverage of DNA was achieved only after nanorod overgrowth. 


\section{Triangular Nanoprism Synthesis, Functionalization, and Purification}

Triangular nanoprisms were synthesized according to Millstone et al. ${ }^{6}$. Whereas DNA functionalization of triangular nanoprisms has already been demonstrated ${ }^{1}$, a typical synthesis includes a significant population of impurity spherical particles. In order to probe only the shape directing effects of the nanoprism, these spherical particles needed to be removed. The assynthesized solution containing both spheres and prisms at $0.05 \mathrm{M} \mathrm{CTAB}$ was functionalized with DNA according to the previously outlined procedure (vide supra). After the final step of the DNA functionalization procedure, addition of an oligonucleotide linker with a ${ }^{5} \mathrm{TGCA}^{3}$ ' "sticky end" resulted in particle aggregation. These aggregates were heated to $55^{\circ} \mathrm{C}$ to dissociate all the particles, then both the sphere $(530 \mathrm{~nm})$ and prism $(900 \mathrm{~nm})$ absorbances were monitored on slow cooling of the solution. Annealing the mixed sphere-prism solutions at a temperature at which prisms reaggregated but spheres remained in solution allowed for selective precipitation of the prisms and subsequent purification by removal of the primarily sphere-containing supernatant. Purified prism samples were washed (6 min, $16100 \mathrm{rcf}$ ) three times in a $0.01 \%$ SDS solution to remove the ${ }^{5} \mathrm{TGCA}^{3}$ ' linkers before being concentrated and assembled using linker oligonucleotides. 


\section{Rhombic Dodecahedra and Octahedra Synthesis and Functionalization}

Rhombic dodecahedra (RD) and octahedra were synthesized using the surfactant cetylpyridinium chloride (CPC) according to the procedure of Niu et al. ${ }^{7}$. We found that all attempts to directly replace CPC with thiolated oligonucleotides resulted in either particle aggregation or a lack of cooperative melting behavior. As a result, CPC-stabilized RDs and octahedra had to first be transferred to CTAB before successful DNA functionalization was observed. The as-synthesized RDs and octahedra were spun down twice (20 min, $1500 \mathrm{rcf}$ ) and resuspended in water to remove excess $\mathrm{CPC}$ surfactant. The resulting particle solution was then brought to $0.05 \mathrm{M} \mathrm{CTAB}$ using a concentrated CTAB solution $(0.2 \mathrm{M})$. After allowing approximately $30 \mathrm{~min}$ for the particles to incubate in the CTAB solution, they could be centrifuged twice (20 min, $830 \mathrm{rcf}$ ) without aggregation and functionalized with thiolated oligonucleotides following the previously outlined protocol (vide supra) (Figure S3).
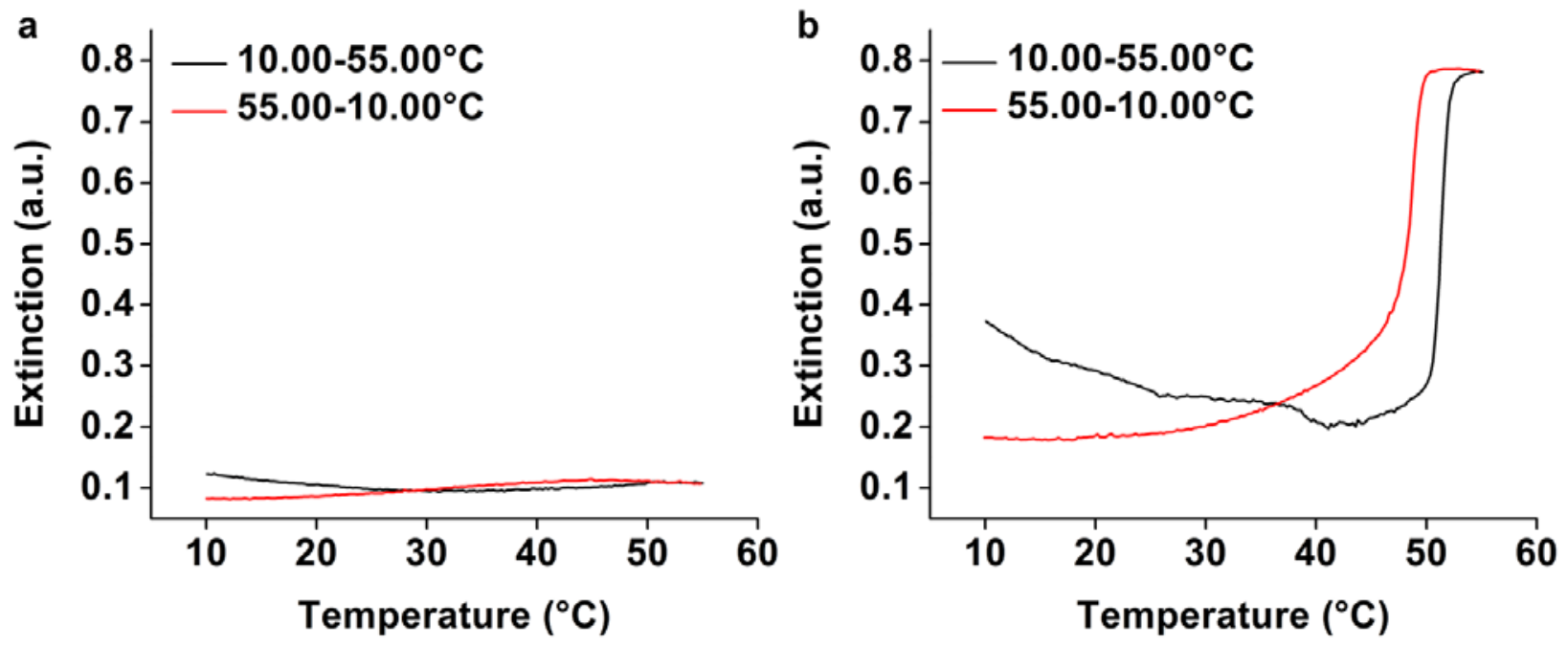

Figure S3. Confirmation of DNA functionalization of rhombic dodecahedra and octahedra. a-b, Extinction monitored at surface plasmon resonance $(618 \mathrm{~nm})$ as a function of temperature for rhombic dodecahedra treated with thiolated DNA and assembled with linker oligonucleotides according to the included procedure. a, Assynthesized rhombic dodecahedra spun down from a CPC solution. b, Rhombic dodecahedra transferred to and spun down from a CTAB solution. The sharp melting transition present only in $\mathbf{b}$ indicates that dense surface coverage of DNA is achieved only if particles are first transferred to CTAB before attempting DNA functionalization. 


\section{Transmission Electron Microscopy Characterization of Anisotropic Nanostructures}

Typically, 10ul of a concentrated nanoparticle solution was drop cast onto a carbon coated copper grid and allowed to dry under vacuum. All images were obtained using a Hitachi 8100 transmission electron microscope operating at $200 \mathrm{kV}$ in bright-field mode (Figure S4).
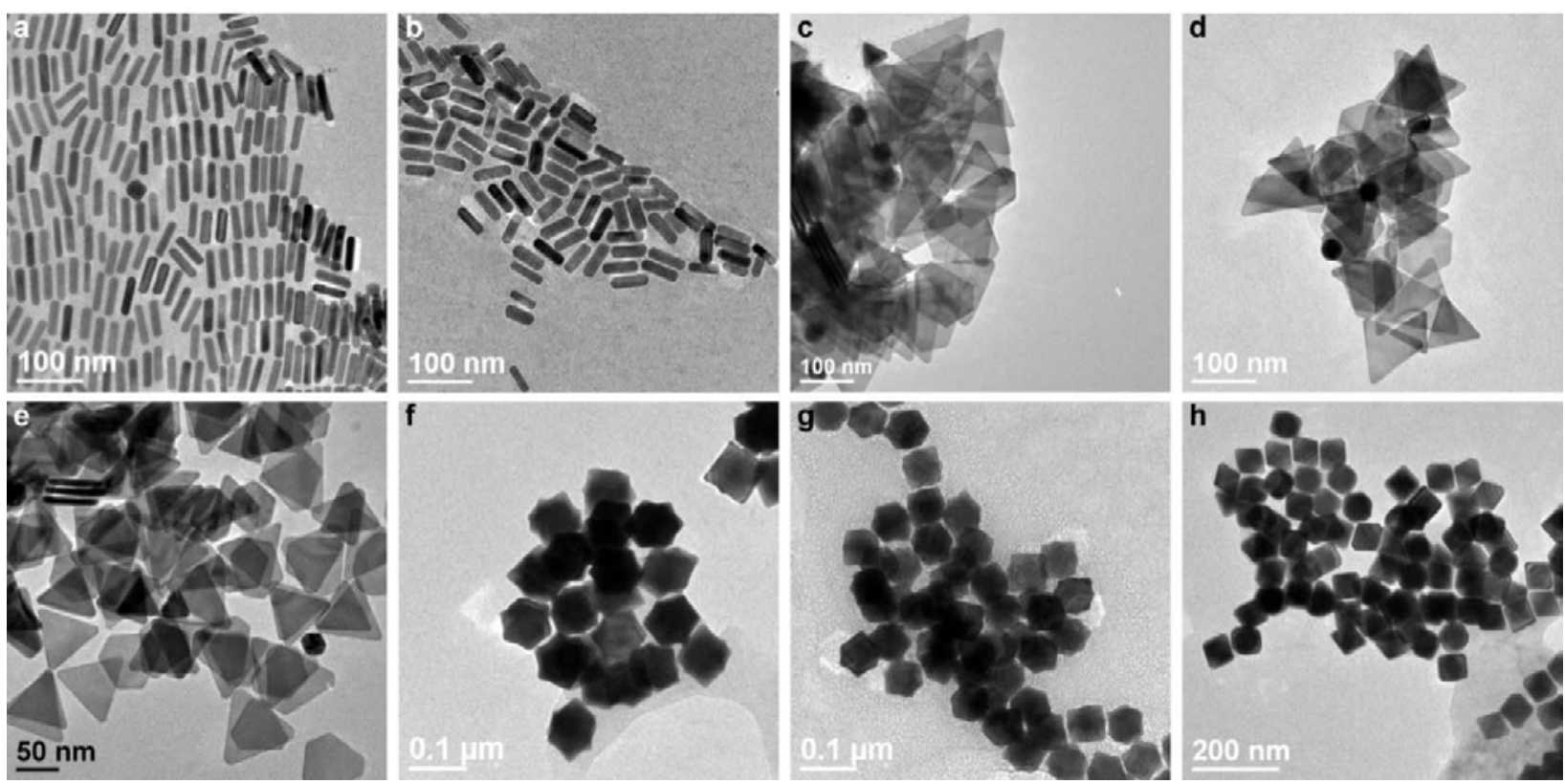

Figure S4. Representative transmission electron microscopy images of each of the anisotropic gold nanostructures synthesized. a, Nanorods $55 \mathrm{~nm}$ length, $14 \mathrm{~nm}$ width. b, Nanorods $52 \mathrm{~nm}$ length, $19 \mathrm{~nm}$ width. c, Triangular nanoprisms $140 \mathrm{~nm}$ edge length, $7 \mathrm{~nm}$ width. d, Triangular nanoprisms $95 \mathrm{~nm}$ edge length, $7 \mathrm{~nm}$ width. e, Triangular nanoprisms $60 \mathrm{~nm}$ edge length, $7 \mathrm{~nm}$ width. f, Rhombic dodecahedra $50 \mathrm{~nm}$ edge length, 81 $\mathrm{nm}$ diameter. g, Rhombic dodecahedra $39 \mathrm{~nm}$ edge length, $64 \mathrm{~nm}$ diameter. h, Octahedra $59 \mathrm{~nm}$ edge length, 83 nm diameter. 


\section{Assembly and Crystallization of Anisotropic Nanostructures}

Nanoparticle superlattices were achieved via combining the DNA-AuNP solutions with linking DNA strands - a detailed description of the exact procedure can be found in reference 8 . The linkers were either purchased from Integrated DNA Technologies or synthesized on an Expedite 8909 Nucleotide Synthesis System (ABI) and all reagents, including 3' C3-thiol phosphoramidites, were obtained from Glen Research; all DNA sequences are shown in Table $\mathrm{S} 1$. Note that DNA linkers labeled as $\mathrm{n}=1-4$ contain a repeating 40-base DNA sequence-prior to adding these linkers to the nanoparticle solutions, the linkers were duplexed to $\mathrm{n}$ equivalents of a 40-base strand complementary to this sequence. This ensured that the DNA linking the nanoparticles was primarily double stranded, which allows for predictability in DNA length and lattice parameters of the resulting crystals ${ }^{9}$. The duplexed linkers were added to the solutions of DNA-AuNPs in a 1:1 ratio with estimates for the number of DNA strands per particle ${ }^{10}$ and allowed to aggregate at room temperature. These solutions were then split and diluted with $0.5 \mathrm{M}$ $\mathrm{NaCl}$ to concentrations appropriate for either small angle X-ray scattering (SAXS) or melting temperature analyses. The melting temperature of each system was performed in a Cary $100 \mathrm{UV}$ Vis, monitoring the change in absorbance at the nanoparticle's predominant plasmon resonance. The solution not used for the melting temperature analysis was then annealed at a temperature $\sim 3-5$ degrees below the melting temperature of the assembled particles for a period of 30 minutes to several hours, depending upon the sample. After annealing, the samples were transferred to $2.0 \mathrm{~mm}$ quartz capillaries and SAXS analysis was performed.

\section{AuNP-Bound DNA ${ }^{5}$ AAG AAT TTA TAA GCA GAA-A ${ }_{10}-\mathrm{C}_{3} \mathrm{SH}^{3^{\prime}}$}

\begin{tabular}{|c|c|}
\hline $\begin{array}{l}\text { Linker DNAs } \\
\qquad \mathbf{n}=\mathbf{0}\end{array}$ & 5'TTC TGC TTA TAA ATT CTT-A-GCGC ${ }^{3 \prime}$ \\
\hline$n=1$ & ${ }^{5}$ TTC TGC TTA TAA ATT CTT-A-(Block Sequence) $)_{1}$-GCGC ${ }^{3}$ \\
\hline$n=2$ & ${ }^{5}$ TTC TGC TTA TAA ATT CTT-A-(Block Sequence) $)_{2}$-GCGC ${ }^{3}$ \\
\hline$n=3$ & ${ }^{5}$ 'TTC TGC TTA TAA ATT CTT-A-(Block Sequence) ${ }_{3}$-GCGC ${ }^{3}$ \\
\hline$n=4$ & ${ }^{5}$ TTC TGC TTA TAA ATT CTT-A-(Block Sequence) $)_{4}-$ GCGC $^{3}$ \\
\hline
\end{tabular}

"Block" Sequence $\quad{ }^{5}$ TTT TTT TTT TTT ACT GAG CAG CAC TGA TTT TTT TTT TTT T-A ${ }^{3{ }^{\prime}}$

\section{Duplexer Strand $\quad{ }^{5}$ AAA AAA AAA AAA ATC AGT GCT GCT CAG TAA AAA AAA AAA A ${ }^{3 '}$}

Table S1. DNA Sequences used to assemble crystals. The AuNP bound DNA was attached to the particle surface via gold-sulfur chemistry. Different DNA lengths were achieved via the use of DNA linkers that contained a variable number of "block sequences". Linkers $n=1,2,3$, and 4 were hybridized to $n$ equivalents of the 40-base duplexer strand (which is complementary to the block sequence) prior to adding the linkers to the nanoparticle solutions. 


\section{Small Angle X-Ray Scattering Characterization of Anisotropic Nanoparticle Superlattices}

SAXS experiments were performed at the DuPont-Northwestern-Dow Collaborative Access Team (DND-CAT) beamline of the Advanced Photon Source (APS) at Argonne National Laboratory. Within the SAXS sample holder, temperature was controlled to within $0.1{ }^{\circ} \mathrm{C}$ during all analyses. X-rays of wavelength $1.24 \AA(10 \mathrm{keV})$ were used to probe the sample, and the scattering angle was calibrated using silver behenate as a standard. Two sets of slits were used to define and collimate the X-ray beam and a pinhole was used to remove parasitic scattering. The $\mathrm{X}$-ray beam cross-section measured $1.0 \times 1.0 \mathrm{~mm}^{2}$, exposure times varied from 0.05 to 1.0 seconds. The scattered radiation was detected with a CCD area detector. Dark current frames were subtracted from all data; scattering of the buffer, DNA, and capillary were negligible compared to the AuNP scattering in these data.

2-D SAXS patterns were then azimuthally averaged and relative scattering intensity was plotted as a function of scattering vector $q$ :

$$
q=4 \pi \sin \theta / \lambda
$$

where $\theta$ is the scattering angle and $\lambda$ is the wavelength of X-ray radiation. Each scan was then divided by the particle's form factor (the predicted scattering pattern for particles that are completely dissociated) and compared against modeled SAXS patterns for ideal nanoparticle superlattice structures. Calculations to obtain both form factor and ideal superlattice scattering plots are discussed later in this text.

Interparticle spacing in these crystals was determined using the following equations:

1. For a 2D HCP array of rods, the interparticle spacing is calculated as

$$
d_{A u}=\left(\frac{2}{\sqrt{3}}\right)\left(\frac{2 \pi}{q_{0}}\right)
$$

where $d_{A u}$ is the distance between the centroid of two nearest neighbor particles and $q_{0}$ is the position of the first order scattering peak.

2. For a linear array of prisms, the interparticle spacing is calculated as

$$
d_{A u}=\frac{2 \pi}{q_{0}}
$$

3. For an FCC arrangement of rhombic dodecahedra, a BCC arrangement of octahedra, and an FCC arrangement of octahedra, the interparticle spacing is calculated as

$$
d_{A u}=\frac{\sqrt{6} \pi}{q_{0}}
$$


Crystal domain sizes were calculated using the Debye-Scherrer equation:

$$
t=\frac{0.9 \lambda}{\beta \cos (\theta)}
$$

where $t$ is the thickness of the crystal in a direction perpendicular to the scattering planes, $\lambda$ is the wavelength of the X-rays, $\beta$ is the radial full-width-half-maximum of the first-order scattering peak and $\theta$ is the scattering angle of the first-order scattering peak. The number of unit cells per crystal for the 3-D lattices was calculated assuming a spherical domain with a diameter equal to the crystal thickness $t$. The number of unit cells per crystal for the 2-D lattices was calculated assuming a circular domain with diameter equal to $t$. The average and standard deviation of these values listed in table 1 in the main text were calculated by averaging the crystal domain size for all assemblies that formed ordered crystals. 


\section{Small Angle X-ray Scattering Data for Anisotropic Nanoparticle Superlattices}

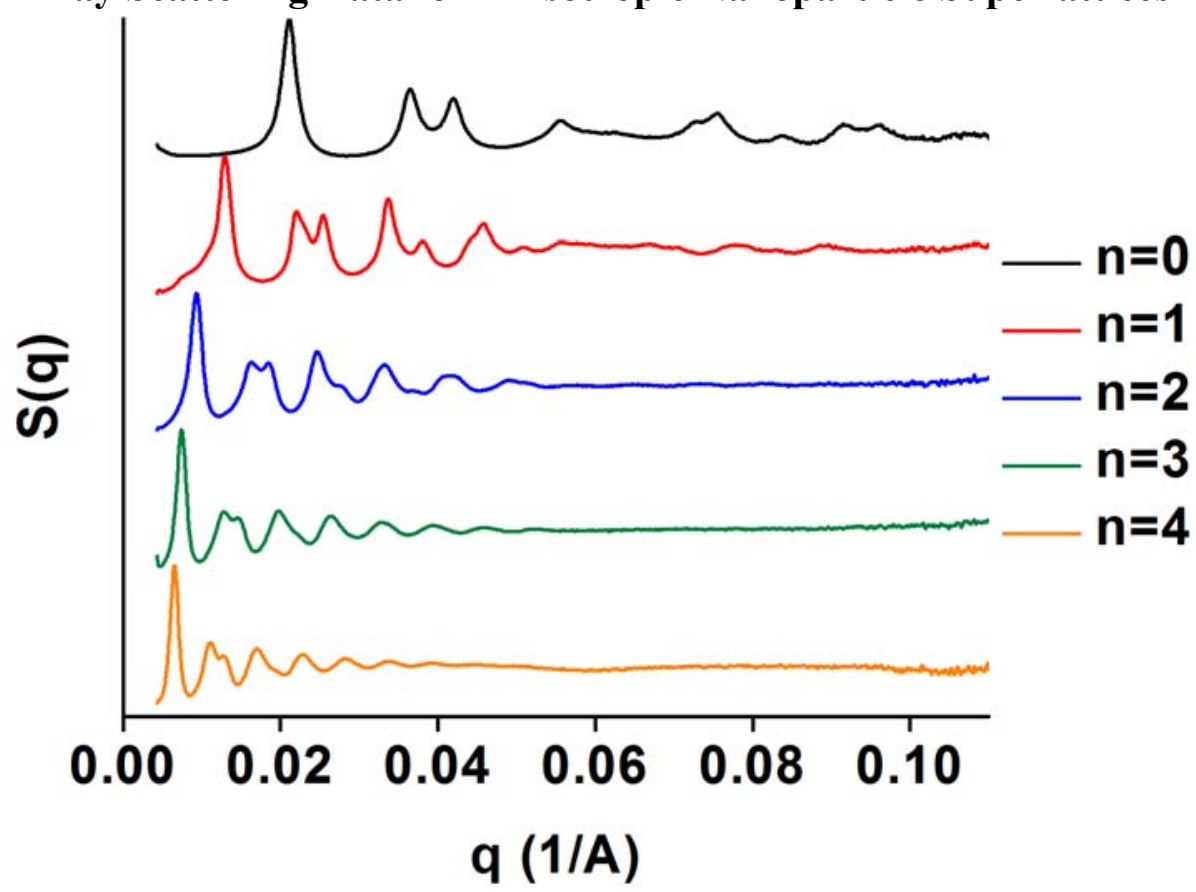

Figure S5. Small angle $X$-ray scattering patterns from crystallized nanorods $(55 \mathrm{~nm}$ length, $14 \mathrm{~nm}$ width) with tunable interparticle distances. Here, " $n$ " refers to the number of 40-base segments present in the linker oligonucleotides used to induce crystallization (See Figure 1d, Table S1) with greater values of $n$ resulting in larger interparticle distances, noted by scattering peaks that are shifted to smaller values of q. All samples index to a $2 \mathrm{D}$ hexagonal lattice, see Table 1 for calculated lattice parameters.

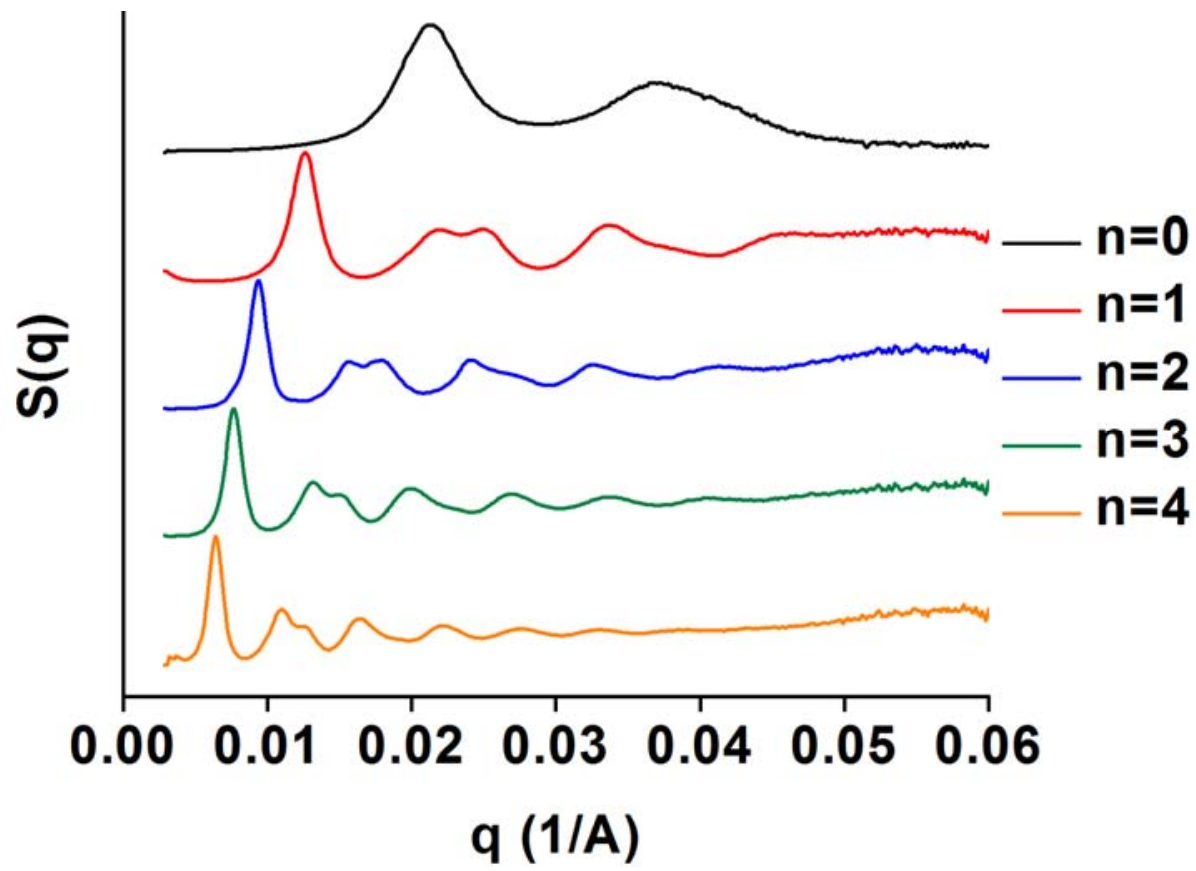

Figure S6. Small angle $X$-ray scattering patterns from crystallized nanorods $(52 \mathrm{~nm}$ length, $19 \mathrm{~nm}$ width) with tunable interparticle distances. Here, " $n$ " refers to the number of 40-base segments present in the linker oligonucleotides used to induce crystallization (See Figure 1d, Table S1) with greater values of $n$ resulting in larger interparticle distances, noted by scattering peaks that are shifted to smaller values of q. All samples index to a 2D hexagonal lattice, see Table 1 for calculated lattice parameters. 


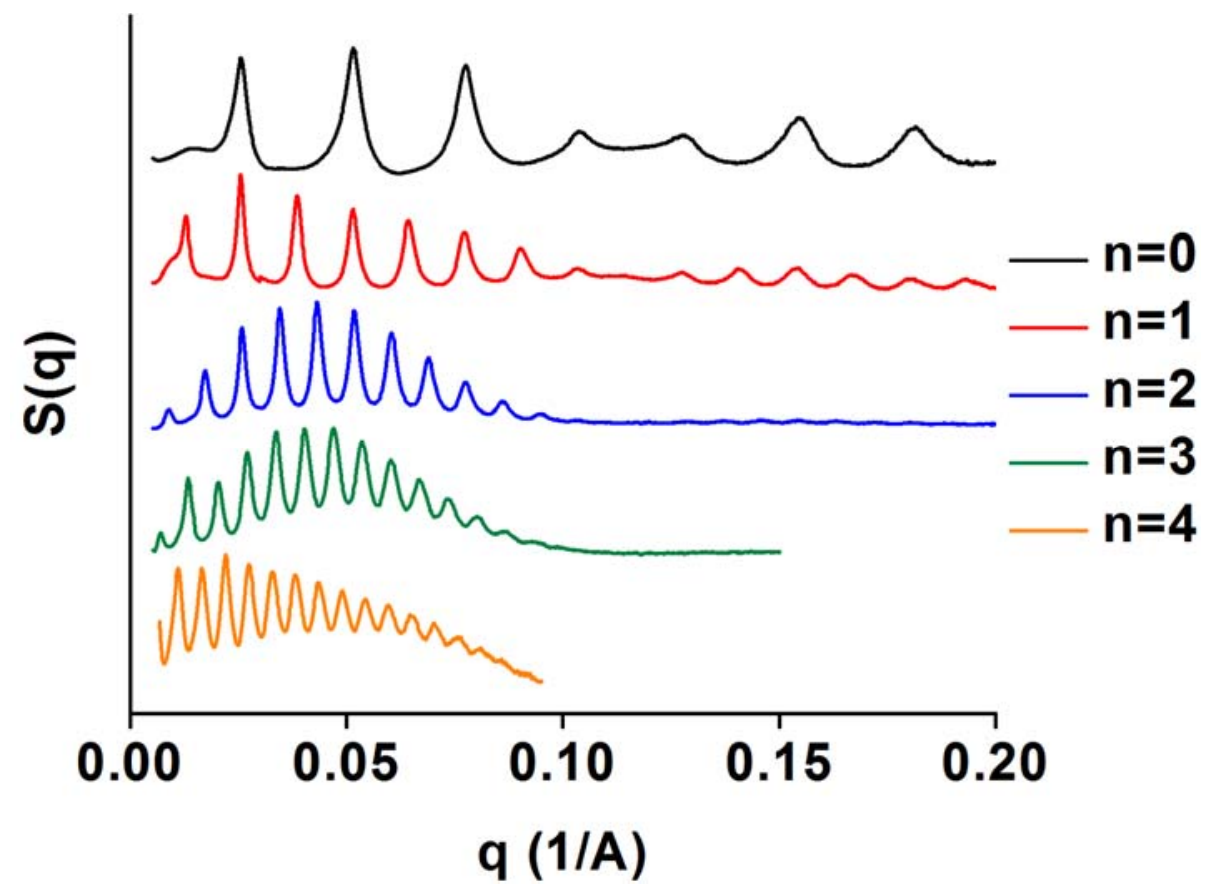

Figure S7. Small angle $X$-ray scattering patterns from crystallized triangular nanoprisms $(140 \mathrm{~nm}$ edge length, $7 \mathbf{~ n m}$ width) with tunable interparticle distances. Here, " $n$ " refers to the number of 40-base segments present in the linker oligonucleotides used to induce crystallization (See Figure 1d, Table S1) with greater values of $\mathrm{n}$ resulting in larger interparticle distances, noted by scattering peaks that are shifted to smaller values of q. All samples index to a $1 \mathrm{D}$ columnar lattice, see Table 1 for calculated lattice parameters.

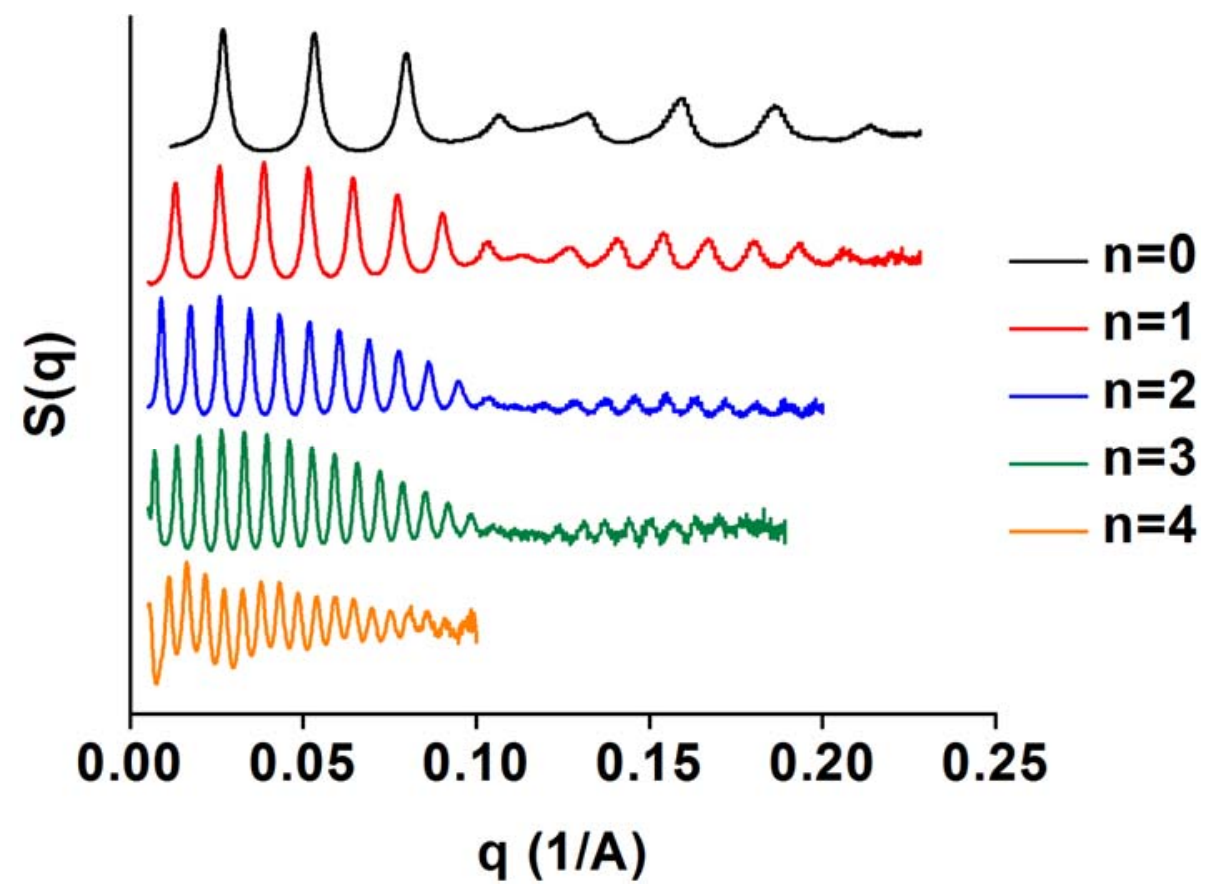

Figure S8. Small angle X-ray scattering patterns from crystallized triangular nanoprisms $(95 \mathbf{~ n m}$ edge length, $7 \mathrm{~nm}$ width) with tunable interparticle distances. Here, " $\mathrm{n}$ " refers to the number of 40-base segments present in the linker oligonucleotides used to induce crystallization (See Figure 1d, Table S1) with greater values of $\mathrm{n}$ resulting in larger interparticle distances, noted by scattering peaks that are shifted to smaller values of q. All samples index to a $1 \mathrm{D}$ columnar lattice, see Table 1 for calculated lattice parameters. 


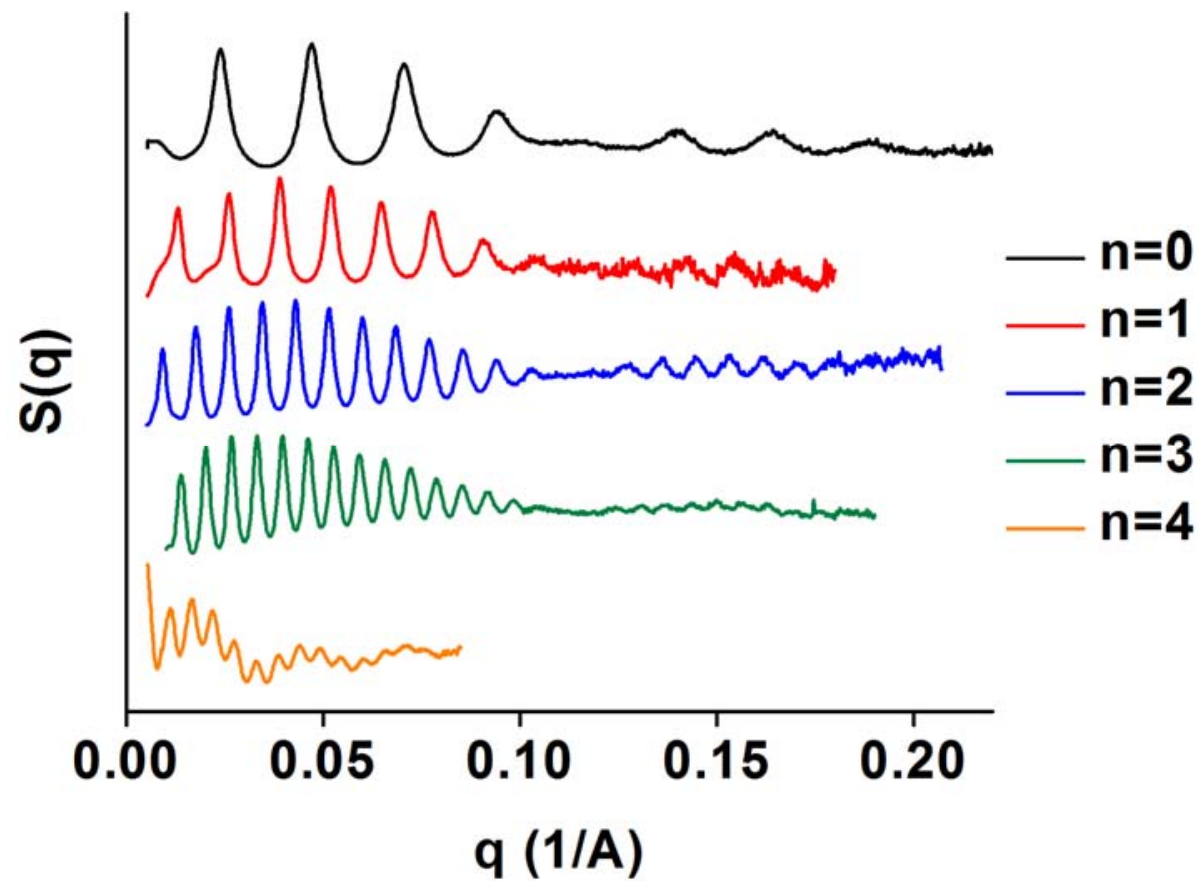

Figure S9. Small angle $X$-ray scattering patterns from crystallized triangular nanoprisms $(60 \mathrm{~nm}$ edge length, $7 \mathrm{~nm}$ width) with tunable interparticle distances. Here, " $\mathrm{n}$ " refers to the number of 40-base segments present in the linker oligonucleotides used to induce crystallization (See Figure 1d, Table S1) with greater values of $\mathrm{n}$ resulting in larger interparticle distances, noted by scattering peaks that are shifted to smaller values of q. All samples index to a 1D columnar lattice, see Table 1 for calculated lattice parameters.

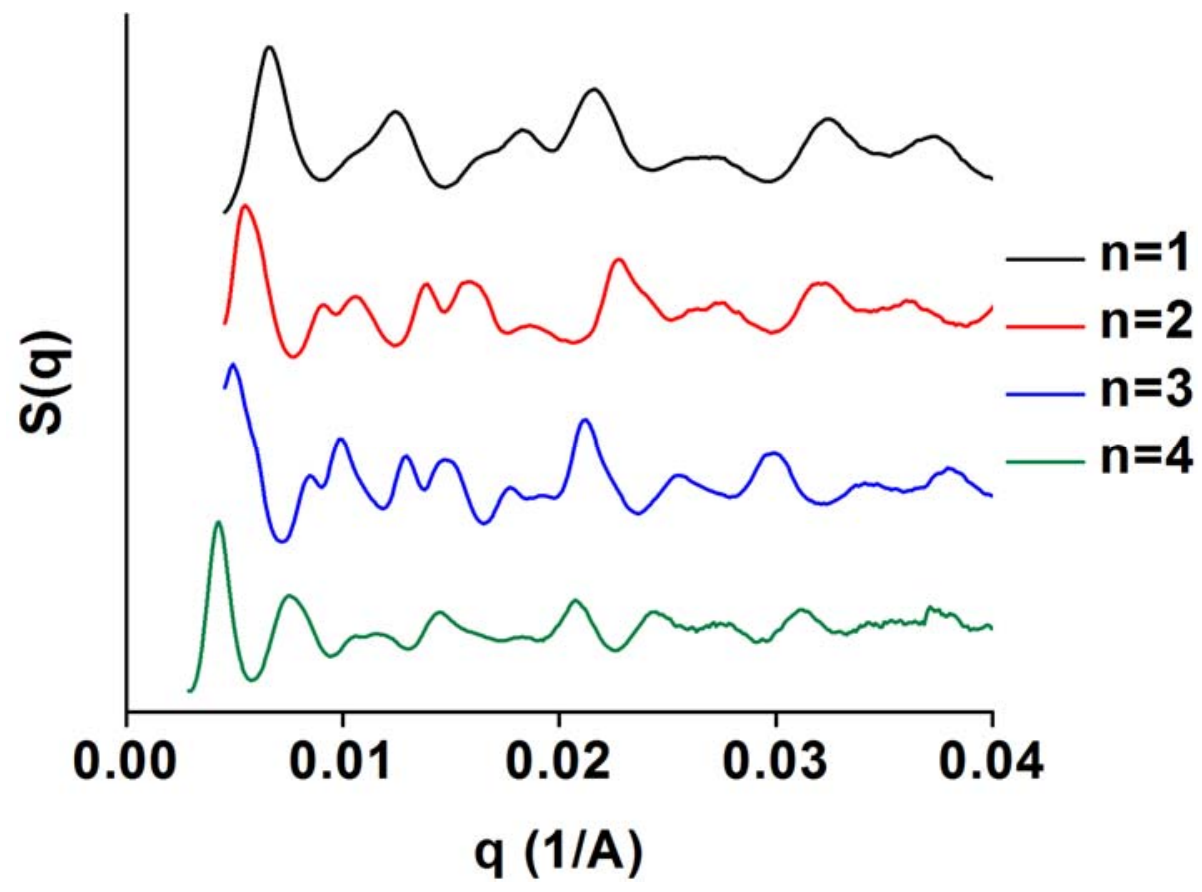

Figure S10. Small angle X-ray scattering patterns from crystallized rhombic dodecahedra $(81 \mathrm{~nm}$ diameter, $50 \mathrm{~nm}$ edge length) with tunable interparticle distances. Here, " $\mathrm{n}$ " refers to the number of 40base segments present in the linker oligonucleotides used to induce crystallization (See Figure 1d, Table S1) with greater values of $\mathrm{n}$ resulting in larger interparticle distances, noted by scattering peaks that are shifted to smaller values of $q$. Only the $n=2$ and $n=3$ samples formed well-ordered FCC lattices; the $n=1$ and $n=4$ structures index to disordered assemblies with no long range order. See Table 1 for calculated lattice parameters. 


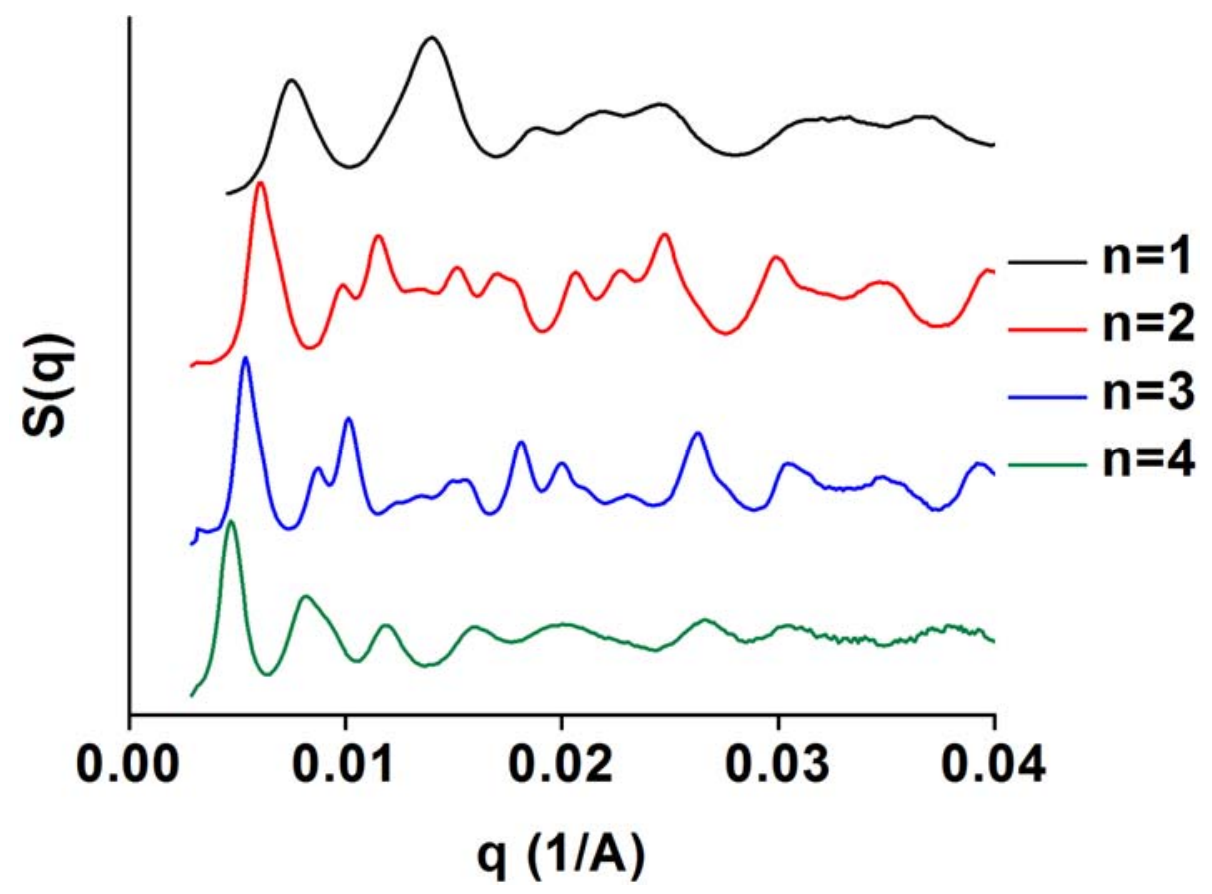

Figure S11. Small angle X-ray scattering patterns from crystallized rhombic dodecahedra (64 $\mathrm{nm}$ diameter, $39 \mathrm{~nm}$ edge length) with tunable interparticle distances. Here, " $\mathrm{n}$ " refers to the number of 40-base segments present in the linker oligonucleotides used to induce crystallization (See Figure 1d, Table S1) with greater values of $\mathrm{n}$ resulting in larger interparticle distances, noted by scattering peaks that are shifted to smaller values of $q$. Only the $n=2$ and $n=3$ samples formed well-ordered FCC lattices; the $n=1$ and $n=4$ structures index to disordered assemblies with no long range order. See Table 1 for calculated lattice parameters.

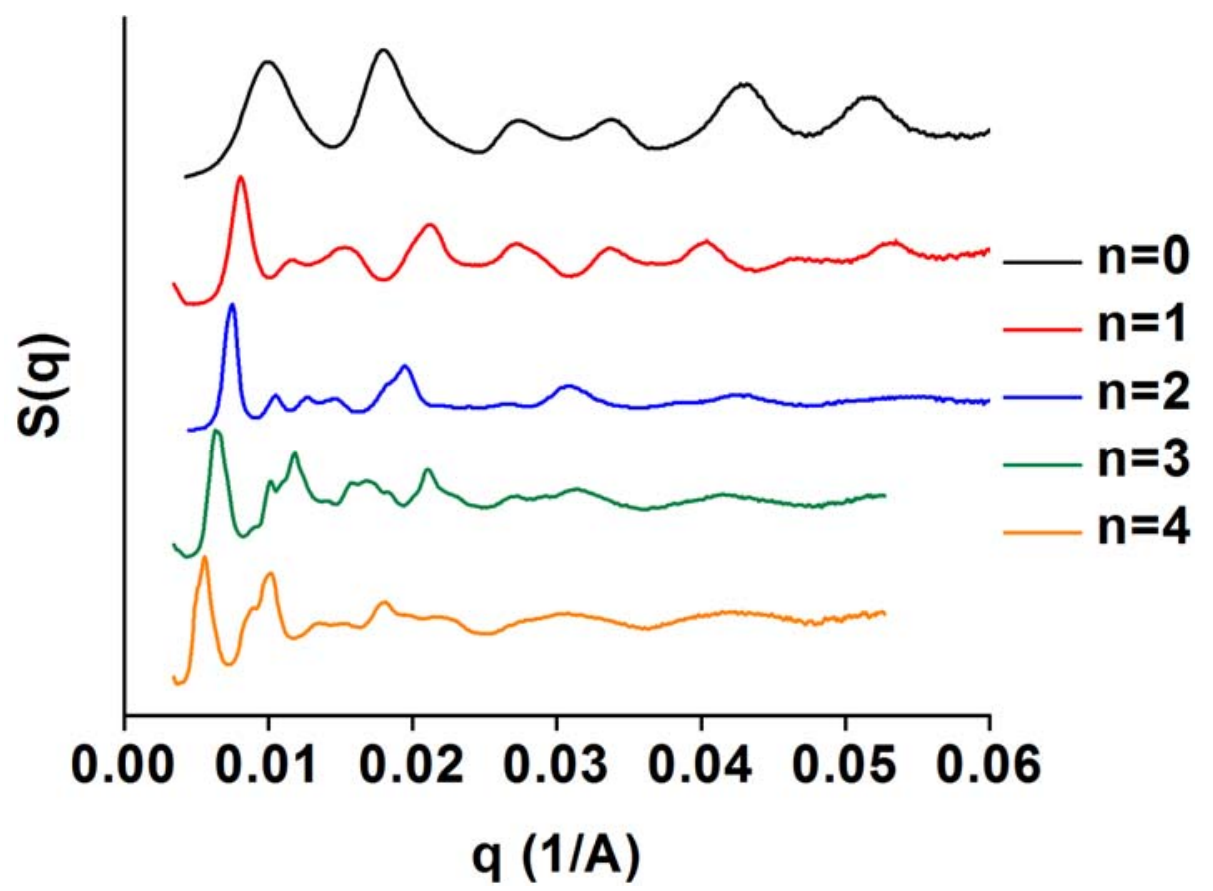

Figure S12. Small angle X-ray scattering patterns from crystallized octahedra (83 $\mathrm{nm}$ diameter, $59 \mathrm{~nm}$ edge length) with tunable interparticle distances. Here, "n" refers to the number of 40-base segments present in the linker oligonucleotides used to induce crystallization (See Figure 1d, Table S1) with greater values of $n$ resulting in larger interparticle distances, noted by scattering peaks that are shifted to smaller values of q. The $\mathrm{n}=0$ sample formed a disordered lattice, the $\mathrm{n}=1$ and $\mathrm{n}=2$ samples formed $\mathrm{BCC}$ lattices, the $\mathrm{n}=4$ sample formed an FCC lattice, and the $\mathrm{n}=3$ sample has both BCC and FCC domains. These lattices are discussed in greater detail later in the supplementary information. 


\section{Transmission Electron Microscopy Characterization of Anisotropic Nanoparticle Superlattices}

Two separate sample preparation methods were used preserve the order of nanoparticle superlattices at the expense of significantly reduced lattice parameters. The first method, which was used to prepare samples of nanoprisms, involved drop-casting a solution of nanoprism superlattices onto a TEM grid and quickly wicking away excess solvent. As has been previously shown, removal of buffer from superlattice solutions results in significant reduction in lattice parameters ${ }^{11}$, but the nanoprisms still retain significant columnar ordering even after removal of solvent (Figure S14). Control experiments confirm that unassembled nanoprisms that are dropcast and quickly dried do not form ordered assemblies, but rather lay flat on the substrate and are typically found as solitary particles. This indicates that the TEM data corroborate the SAXS data, and that the organization of nanoprisms into 1-D stacks is preserved after drying of the sample.

The second method, utilized to prepare samples of nanorods (Figure S13), rhombic dodecahedra (Figure S15), and octahedra (Figure S16), involved embedding the samples in a resin which could be sectioned and imaged using standard biological TEM techniques. First, the solutions of nanoparticles were repeatedly washed with $0.5 \mathrm{M} \mathrm{NaNO}_{3}$ in order to remove all chloride anions from solution. This was followed by the addition of a solution of $1 \mathrm{M} \mathrm{AgNO}_{3}$; the transfer into a solution of $\mathrm{NaNO}_{3}$ prevented any precipitation of insoluble $\mathrm{AgCl}$ upon the addition of $\mathrm{AgNO}_{3}$. An aliquot of $0.1 \mathrm{M} \mathrm{NaBH}_{4}$ was then added to this solution, resulting in instantaneous precipitation of insoluble silver solid. The presence of these large Ag particles provided a handle for centrifugation, embedding, and general manipulation of the nanoparticle superlattices and was found to be necessary to achieve high quality images. The solution was then centrifuged and the buffer was replaced with ethanol resulting in collapse of the DNA ${ }^{12}$ while maintaining the symmetry of the colloidal crystals.

Once in ethanol, these samples were embedded in EMBed-812 resin (Electron Microscopy Sciences) following standard protocols provided by the manufacturer. The resin was polymerized at $60{ }^{\circ} \mathrm{C}$ for 16-20 hours to solidify, then cooled and sectioned with a diamond knife and an ultramicrotome. The resulting sections varied in thickness between $\sim 60$ and $\sim 250 \mathrm{~nm}$. TEM images were obtained on a Hitachi HD-2300 transmission electron microscope in Zcontrast mode. These images were then compared with modeled lattices to verify that the particles had indeed retained their ordering, even though their lattice parameters had been significantly reduced. 

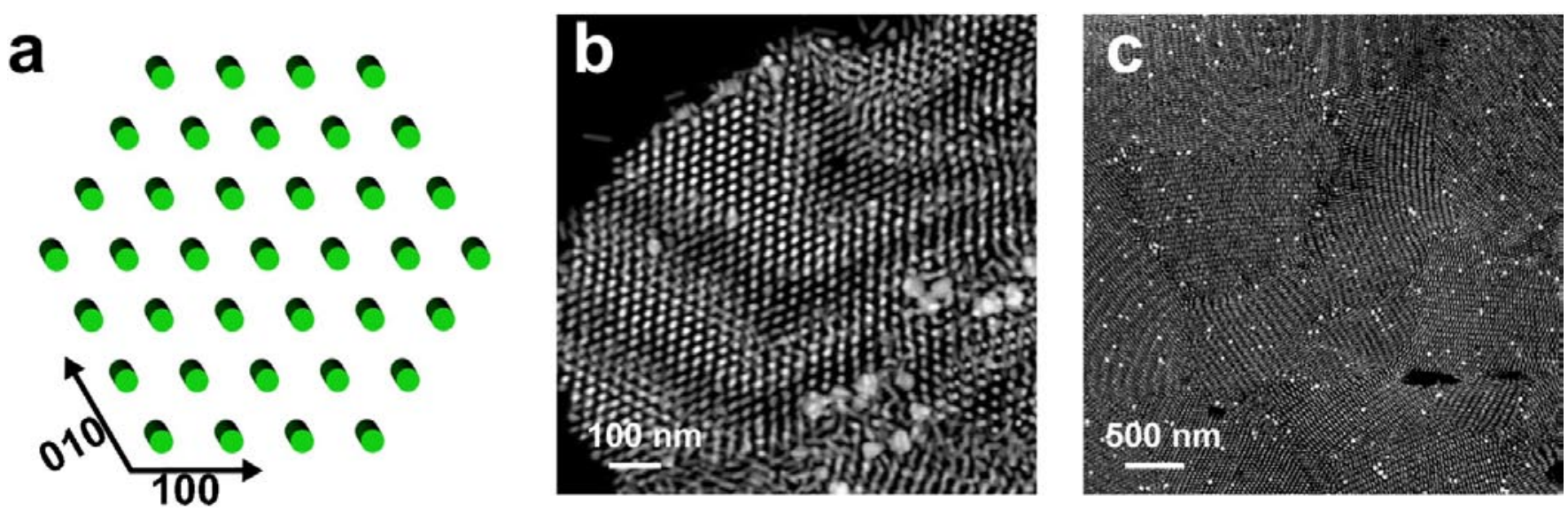

Figure S13. Representative TEM images of ordered nanorod superlattices. a, Schematic illustration of the orientation of crystallized nanorods (55 nm length, $14 \mathrm{~nm}$ width) shown in part b. b, High magnification image of nanorods in a hexagonal arrangement. c, Low magnification image of ordered nanorod superlattices. In both images, sections are $\sim 100 \mathrm{~nm}$ thick. Both images correspond to the sample represented in Figure $2 \mathrm{~b}$.
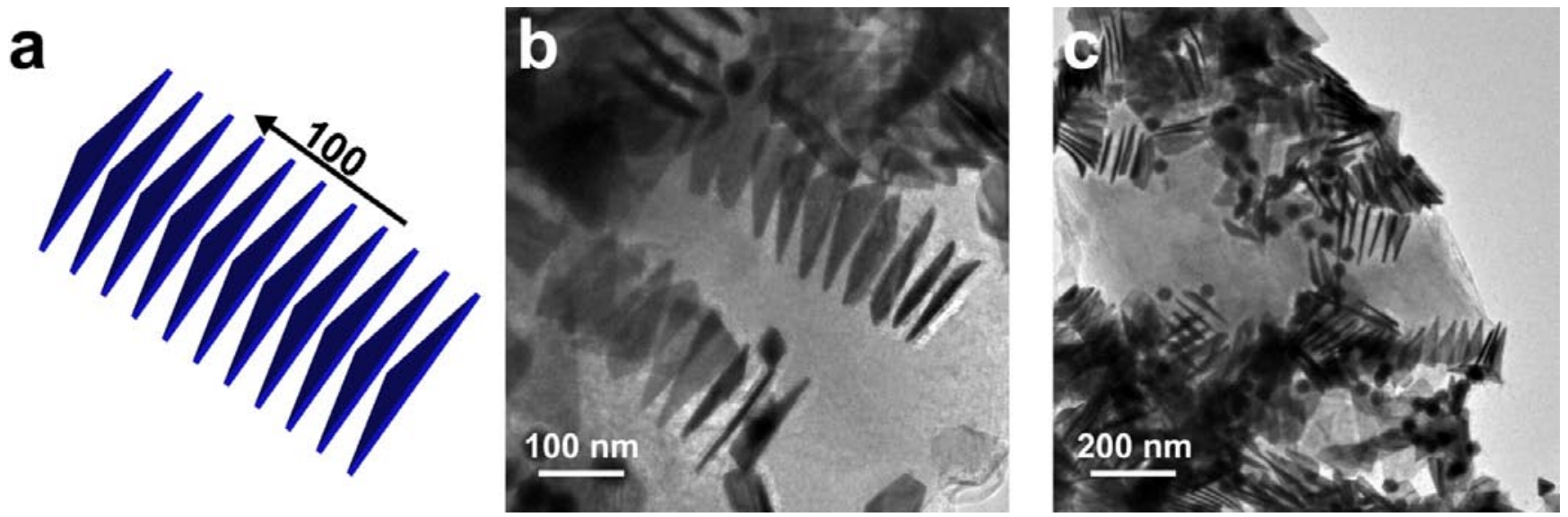

Figure S14. Representative TEM images of ordered nanoprism superlattices. a, Schematic illustration of the orientation of crystallized nanoprisms (140 nm edge length) found in part $b$. b, High magnification image of nanoprisms in a columnar arrangement. c, Low magnification image of ordered nanoprism superlattices. Both images correspond to the system represented in Figure S7, n=1 linker. 

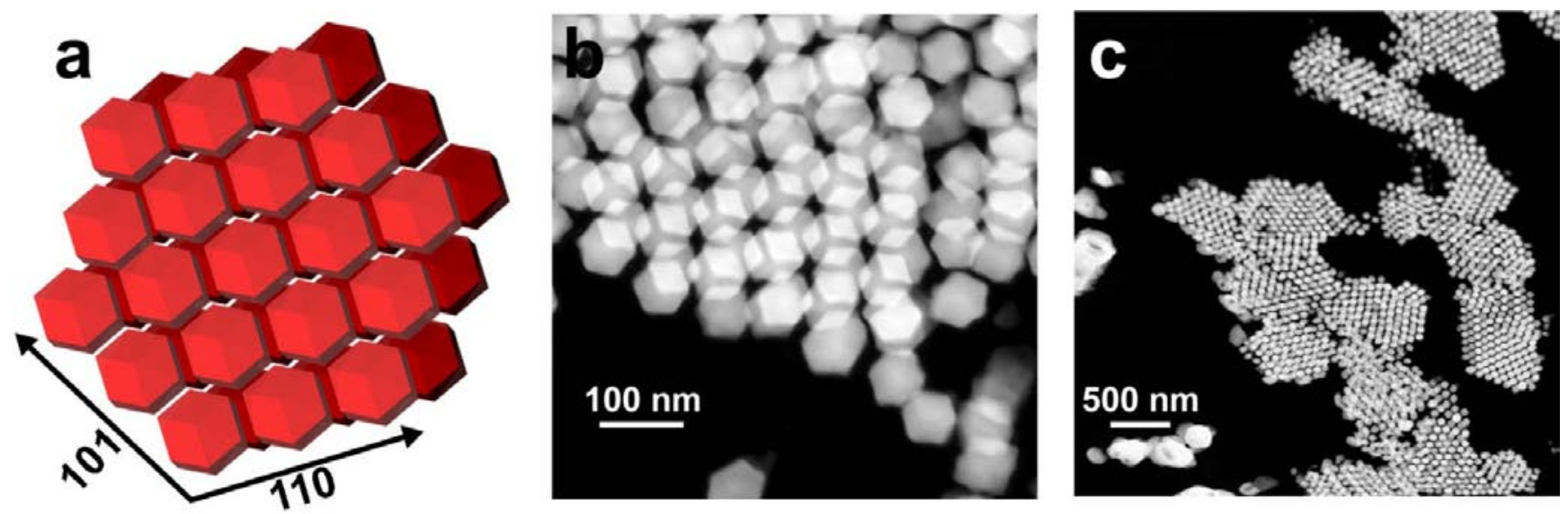

Figure S15. Representative TEM images of ordered rhombic dodecahedra superlattices. a, Schematic illustration of the orientation of crystallized rhombic dodecahedra (64 nm diameter) found in part b. b, High magnification image of rhombic dodecahedra in an FCC arrangement. c, Low magnification image of ordered rhombic dodecahedra superlattices. In both TEM images, sections are $\sim 180 \mathrm{~nm}$ thick. Both images correspond to the sample represented in Figure $2 \mathrm{f}$.
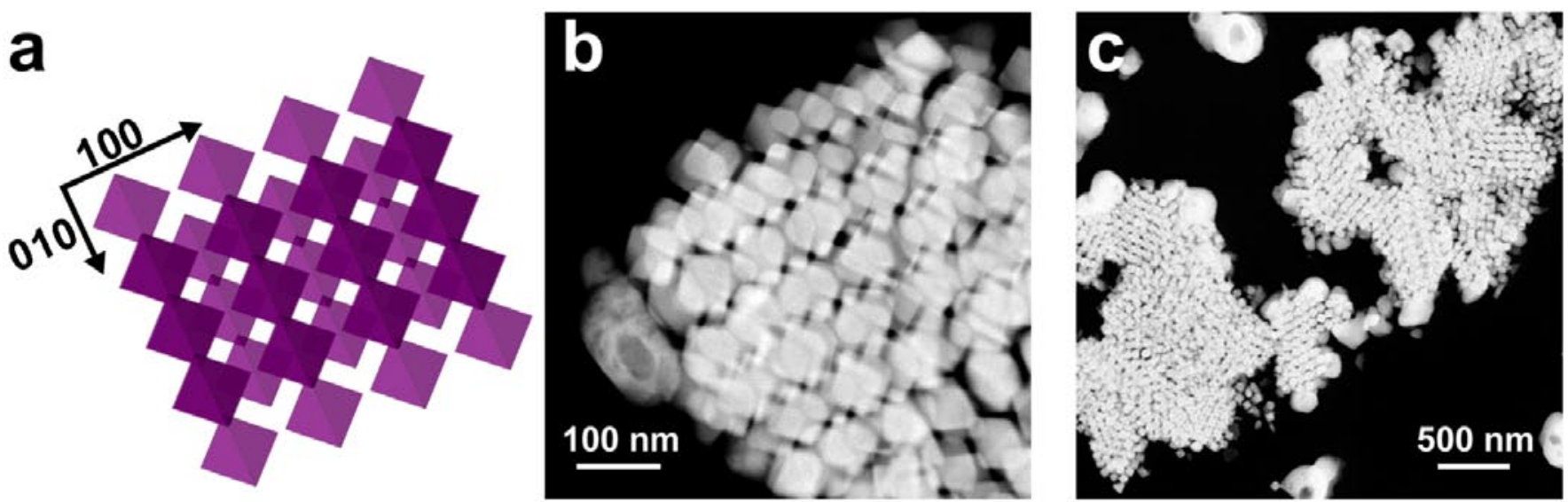

Figure S16. Representative TEM images of ordered octahedra superlattices. a, Schematic illustration of the orientation of crystallized octahedra ( $83 \mathrm{~nm}$ diameter) found in part $\mathbf{b}$. $\mathbf{b}$, High magnification image of octahedra in a BCC arrangement. c, Low magnification image of ordered octahedra superlattices. In part b sections are $\sim 150 \mathrm{~nm}$ thick, and in part c sections are $\sim 250 \mathrm{~nm}$ thick. Both images correspond to the sample represented in Figure 3c. 


\section{Modeling and Simulation of SAXS Patterns of Anisotropic Nanoparticle Superlattices}

The $\mathrm{x}$-ray scattering intensity of randomly oriented crystals is calculated as:

$$
\begin{aligned}
I(q) & =\left\langle|F(\mathbf{q})|^{2} S(\mathbf{q})\right\rangle \\
& =P(q)\left\langle\frac{|F(\mathbf{q})|^{2}}{P(q)} S(\mathbf{q})\right\rangle, \\
& =P(q) S(q)
\end{aligned}
$$

where the bracket denotes the orientational average, $P(q)=\left\langle|F(\mathbf{q})|^{2}\right\rangle$ is the form factor intensity of a randomly oriented polyhedron, and $q$ is the modulus of the scattering vector $\mathbf{q}$. The structure factor of a randomly oriented crystal $S(q)$ is defined as:

$$
\left\langle\frac{|F(\mathbf{q})|^{2}}{P(q)} S(\mathbf{q})\right\rangle \equiv S(q) .
$$

The form factor amplitude of any arbitrary object is calculated as:

$$
F(\mathbf{q})=\int_{V} e^{j \mathbf{q} \cdot \mathbf{r}} d \mathbf{r}
$$

where the electron densities inside and outside the object are 1 and 0 , respectively. 
The form factor amplitudes of various polyhedra used in this work are derived as follows ${ }^{13}$ :

1. The form factor of a pyramid

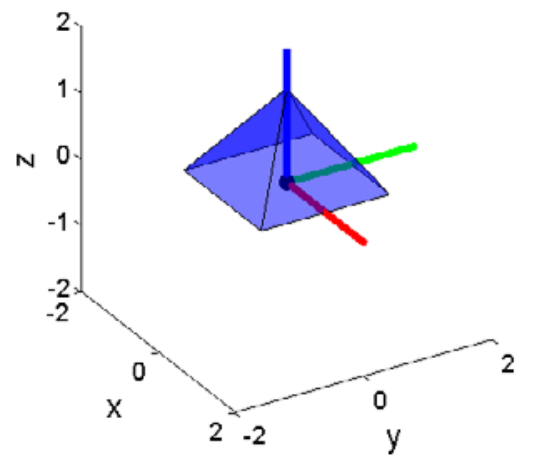

where the length of a bottom edge: $2 R$; the height of the pyramid: $H$

$$
\begin{aligned}
F_{p y}(\mathbf{q}, R, H) & =\frac{H}{q_{x} q_{y}}\left\{\cos \left[\left(q_{x}-q_{y}\right) R\right] K_{1}+\sin \left[\left(q_{x}-q_{y}\right) R\right] K_{2}-\cos \left[\left(q_{x}+q_{y}\right) R\right] K_{3}-\sin \left[\left(q_{x}+q_{y}\right) R\right] K_{4}\right\} \\
K_{1} & =\sin _{c}\left(q_{1} H\right) e^{i q_{1} H}+\sin _{c}\left(q_{2} H\right) e^{-i q_{2} H} \\
K_{2} & =-i \sin _{c}\left(q_{1} H\right) e^{i q_{1} H}+i \sin _{c}\left(q_{2} H\right) e^{-i q_{2} H} \\
K_{3} & =\sin _{c}\left(q_{3} H\right) e^{i q_{3} H}+\sin _{c}\left(q_{4} H\right) e^{-i q_{4} H} \\
K_{4} & =-i \sin _{c}\left(q_{3} H\right) e^{i q_{3} H}+i \sin _{c}\left(q_{4} H\right) e^{-i q_{4} H} \\
q_{1} & =\frac{1}{2}\left[\frac{q_{x}-q_{y}}{\sqrt{2}}+q_{z}\right] \\
q_{2} & =\frac{1}{2}\left[\frac{q_{x}-q_{y}}{\sqrt{2}}-q_{z}\right] \\
q_{3} & =\frac{1}{2}\left[\frac{q_{x}+q_{y}}{\sqrt{2}}+q_{z}\right] \\
q_{4} & =\frac{1}{2}\left[\frac{q_{x}+q_{y}}{\sqrt{2}}-q_{z}\right] \\
V_{p y} & =\frac{4}{3} H R^{2}
\end{aligned}
$$

$\sin _{c}(x)=\sin (x) / x$

$J_{1}:$ The Bessel function of first order. 
2. The form factor of a cube where the edge length: $2 R$

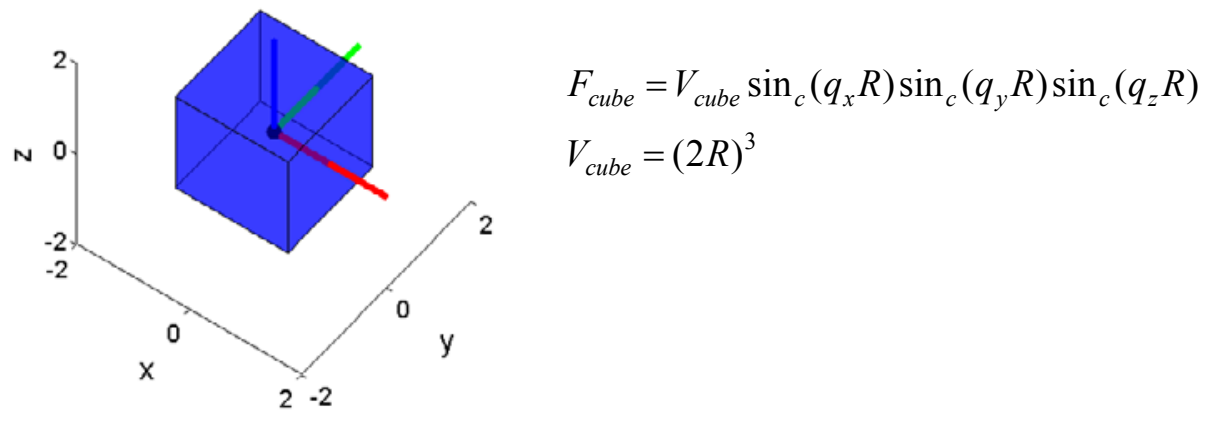

3. The form factor of a cylinder where the radius: $R$, length: $H$

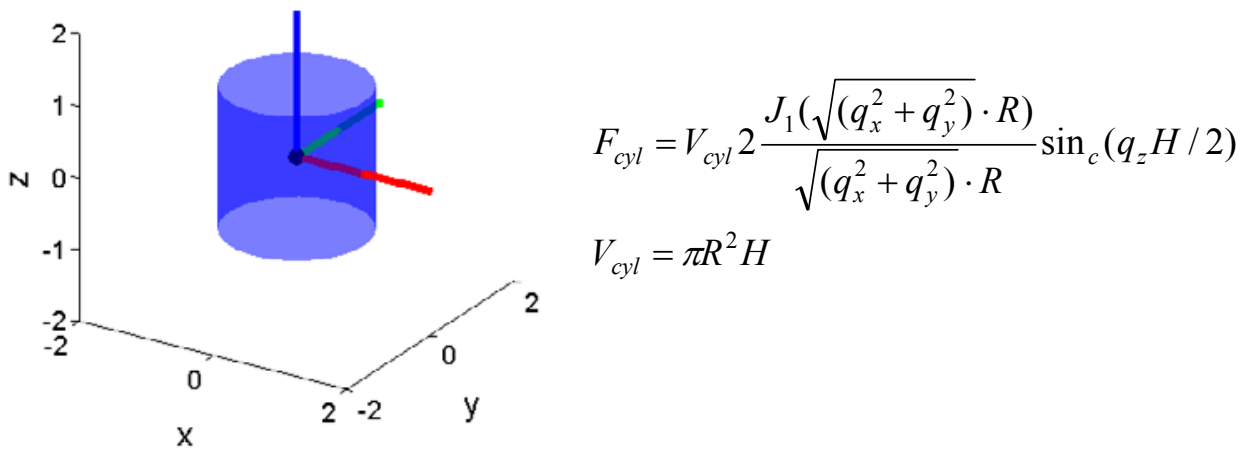

4. The form factor of an octahedron where the edge length: $2 R$

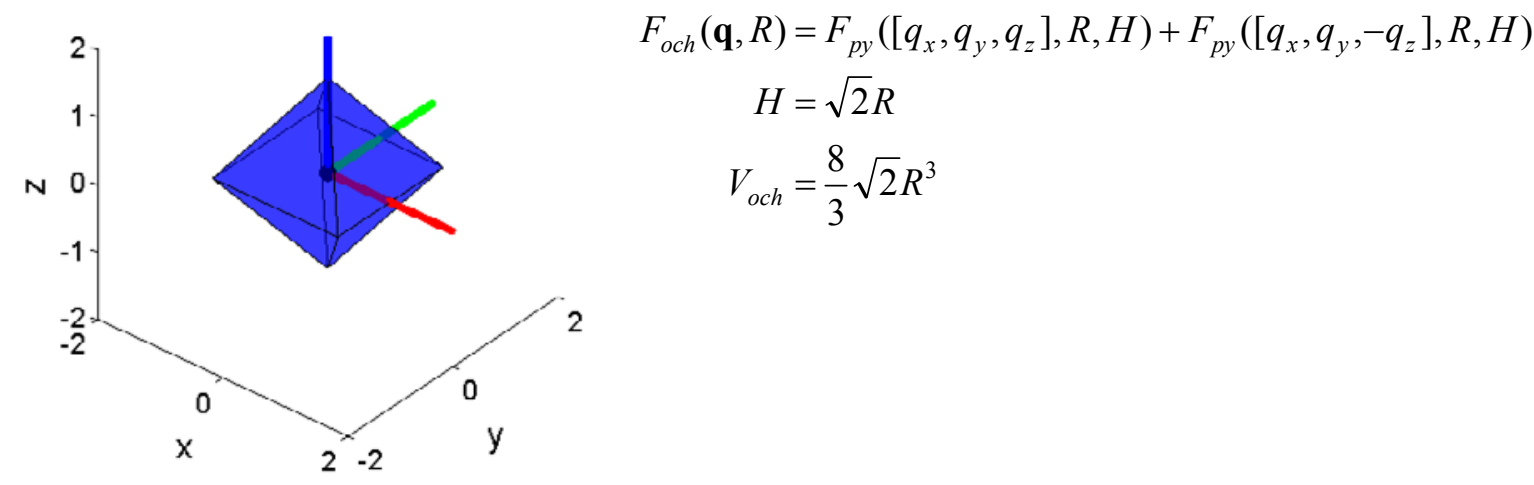


5. The form factor of a rhombic dodecahedron (RD) where the edge length: $\sqrt{3} R$

Note: A rhombic dodecahedraon is composed of 6 pyramids with an edge length of $2 R$ and height $R$ and one cube with an edge length of $2 R$. A rhombic dodecahedron is constructed by aligning the base of each pyramid with the faces of the cube.

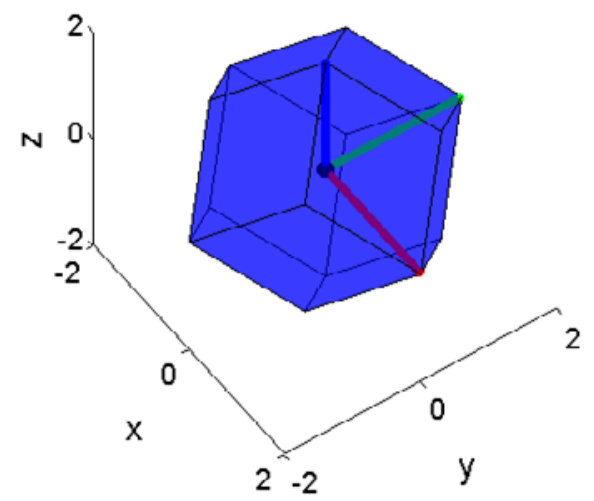

$$
\begin{aligned}
F_{R D}(\mathbf{q}, R) & =\left(\begin{array}{l}
F_{p y}\left(\left[q_{x}, q_{y}, q_{z}\right], R, R\right) e^{j q_{z} R}+F_{p y}\left(\left[q_{x}, q_{y},-q_{z}\right], R, R\right) e^{-j q_{z} R} \\
+F_{p y}\left(\left[q_{x}, q_{z}, q_{y}\right], R, R\right) e^{j q_{y} R}+F_{p y}\left(\left[q_{x}, q_{z},-q_{y}\right], R, R\right) e^{-j q_{y} R} \\
+F_{p y}\left(\left[q_{z}, q_{y}, q_{x}\right], R, R\right) e^{j q_{x} R}+F_{p y}\left(\left[q_{z}, q_{y},-q_{x}\right], R, R\right) e^{-j q_{x} R} \\
+F_{\text {cube }}\left(\left[q_{x}, q_{y}, q_{z}\right], R\right)
\end{array}\right) \\
V_{R D}= & 6 V_{p y}+V_{\text {cube }} \\
= & 16 R^{3}
\end{aligned}
$$

6. The form factor of a prism

where the edge length: $2 R$, height: $H$

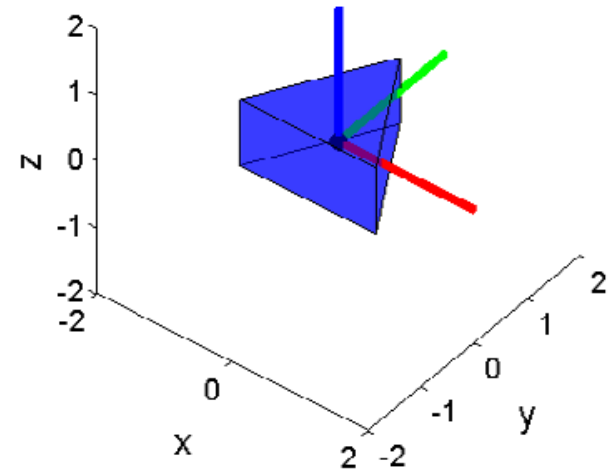

$$
\begin{aligned}
F_{\text {prism }}(\mathbf{q}, R, H) & =\frac{2 \sqrt{3} e^{-j q_{y} R / \sqrt{3}} H}{q_{x}\left(q_{x}^{2}-3 q_{y}^{2}\right)}\left(q_{x} e^{j q_{y} R \sqrt{3}}-q_{x} \cos \left(q_{x} R\right)-j \sqrt{3} q_{y} \sin \left(q_{x} R\right)\right) \sin _{c}\left(q_{z} H / 2\right) \\
V_{\text {prism }} & =\sqrt{3} R^{2} H
\end{aligned}
$$


The form factor intensity of a randomly oriented polyhedron $P(q)$ is calculated as:

$$
P(q)=\int_{0}^{2 \pi} \int_{0}^{\pi}|F(-q \sin \theta \cos \varphi, q \sin \theta \sin \varphi, q \cos \theta)|^{2} \sin \theta d \theta d \varphi
$$

For this work the structure factor of a randomly oriented crystal is obtained experimentally

$$
S(q)_{\text {meas }}=\frac{I(q)_{\text {meas }}}{P(q)_{\text {meas }}}
$$

and theoretically

$$
S(q)_{\text {calc }}=\frac{c}{q_{h k l}^{2} P\left(q_{h k l}\right)_{\text {calc }}} \sum_{\{h k l\}}^{m_{h k l}}\left|F\left(R \cdot \mathbf{q}_{h k l}\right) \sum_{i=1}^{n_{c}} e^{2 \pi j\left(x_{i} h+y_{i} k+z_{i} l\right)}\right|^{2} e^{-\sigma_{D}^{2} q_{h k l}^{2} a^{2}} \frac{\sigma_{L} /(2 \pi)}{\left(q-q_{h k l}\right)^{2}+\left(\sigma_{L} / 2\right)^{2}} .
$$

Experimental $P(q)_{\text {meas }}$ are obtained by raising the temperature of the DNA-NP solutions to a temperature at which the nanoparticles dissociate (typically $>55^{\circ} \mathrm{C}$ ). In the calculation of the structure factor, $1 / q_{h k l}^{2}$ is the Lorentz factor and $c$ is a constant. $P\left(q_{h k l}\right)_{\text {calc }}$ is the calculated form factor intensity for a randomly oriented particle at $q=q_{h k l}$ for $\{h k l\}$ reflections. $F\left(R \cdot \mathbf{q}_{h k l}\right)$ is the amplitude of the form factor for an oriented particle in a unit cell. $M$ is the rotation matrix for a particle in a unit cell. In this work, Rodrigues' rotation formula is used. $m_{h k l}$ is the multiplicity of a $\{h k l\} .\left(x_{i}, y_{i}, z_{i}\right)$ is the fractional coordinate of $i$ th of $n_{c}$ particles in a unit cell. $e^{-\sigma_{D}^{2} q_{h k l}^{2} a^{2}}$ is the Debye-Waller factor or lattice disorder factor, where $\sigma_{D}$ and $a$ are the disorder parameter and the nearest neighbor distance, respectively. In this work, $\sigma_{D}$ less than 0.01 is used. Peak shape is approximated with the Lorentz function, $\frac{\sigma_{L} /(2 \pi)}{\left(q-q_{h k l}\right)^{2}+\left(\sigma_{L} / 2\right)^{2}}$, where $\sigma_{L}$ is the width of Lorentz function. In this work, $\sigma_{L}=5 \cdot 10^{-3}$. 


\section{Example Form Factor Analysis of Selected Anisotropic Nanostructures}

The form factor for aspect ratio 4 nanorods used in this work:

$\mathrm{L}=500 \AA, \mathrm{R}=62.5 \AA$, and polydispersity $12 \%$

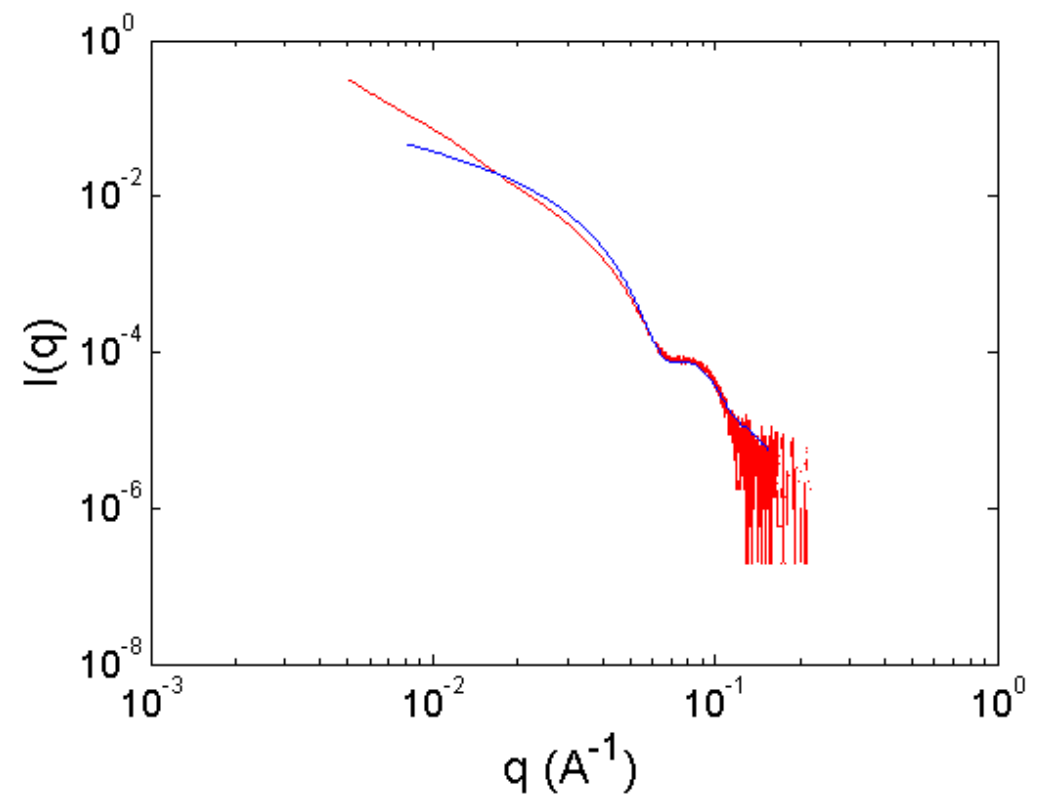

Figure S17. Example of form factor fitting for nanorods. The red line is the experimentally obtained form factor, and the blue line represents the modeled form factor.

The form factor for triangular nanoprisms used in this work:

$\mathrm{E}=500 \AA, \mathrm{W}=58 \AA$, and polydispersity $5 \%$

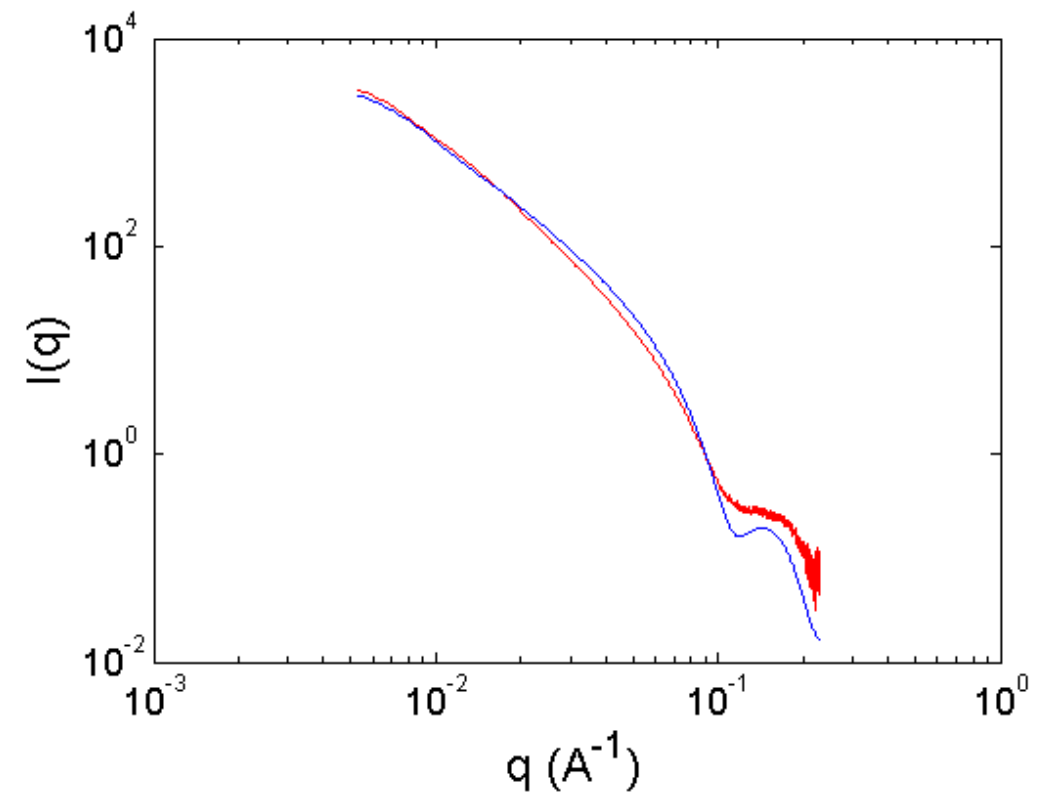

Figure S18. Example of form factor fitting for triangular prisms. The red line is the experimentally obtained form factor, and the blue line represents the modeled form factor. 
The form factor for rhombic dodecahedra used in this work:

$\mathrm{R}=210.3$, polydispersity $6 \%$

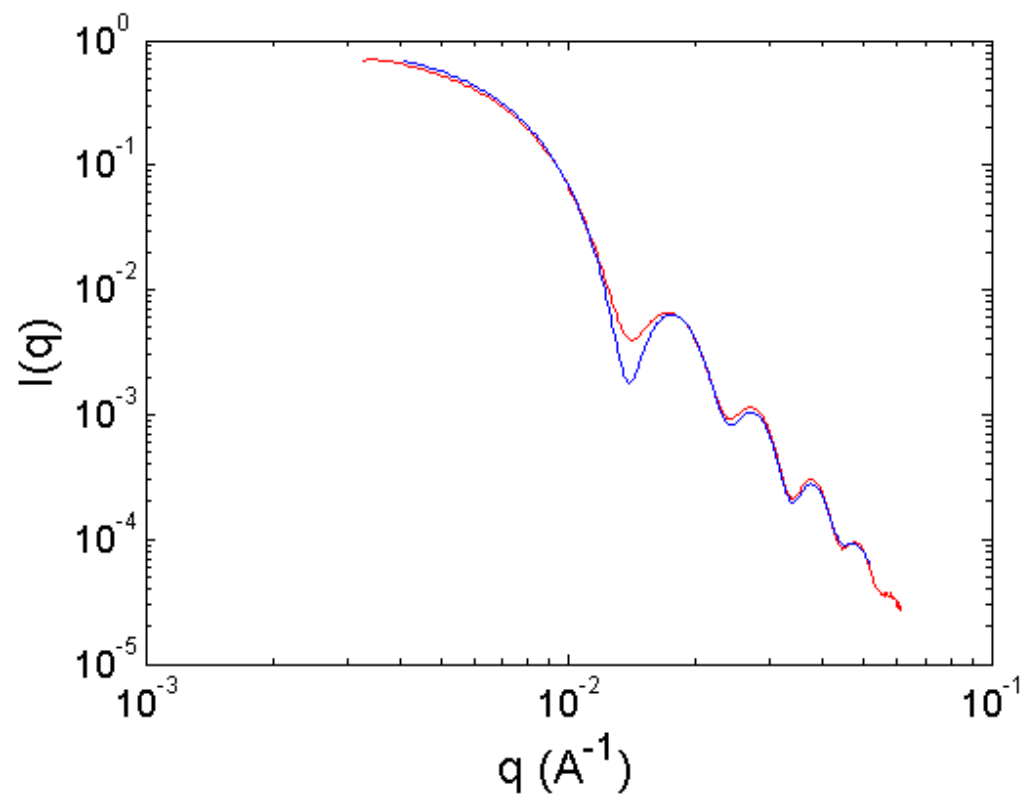

Figure S19. Example of form factor fitting for rhombic dodecahedra. The red line is the experimentally obtained form factor, and the blue line represents the modeled form factor.

The form factor for octahedra used in this work:

$\mathrm{R}=327.87$, polydispersity $6 \%$

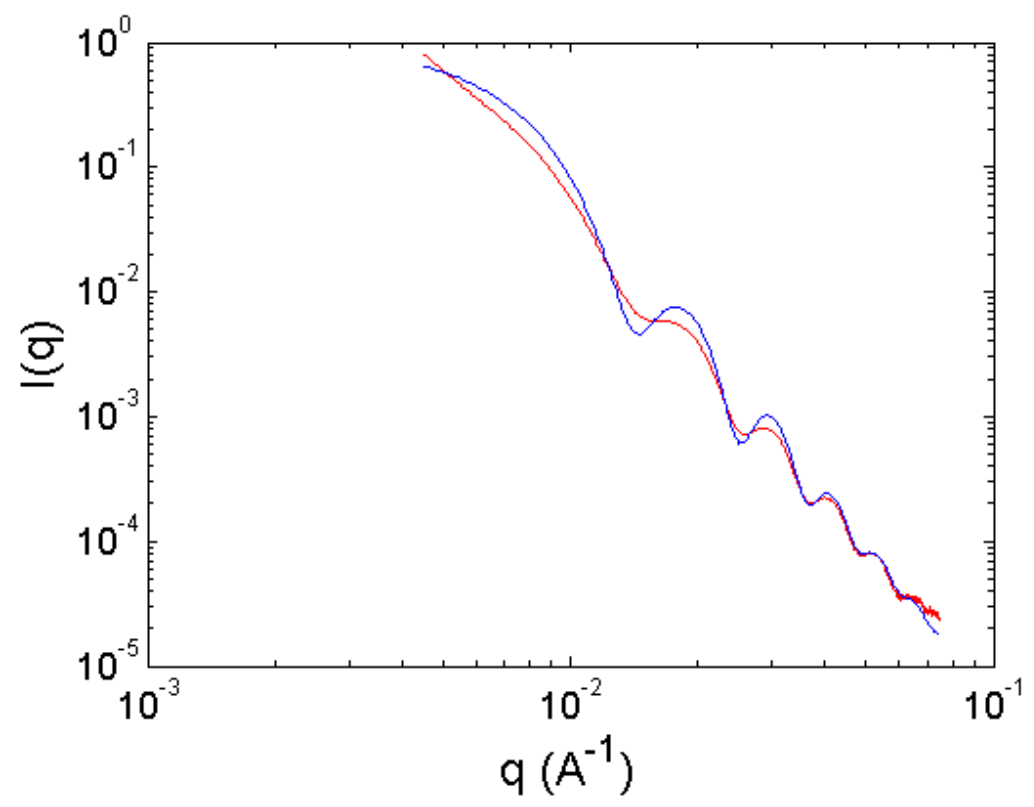

Figure S20. Example of form factor fitting for octahedra. The red line is the experimentally obtained form factor, and the blue line represents the modeled form factor. 


\section{Supplementary Discussion}

\section{Evidence of Primarily 2-D Ordering in Superlattices of Gold Nanorods}

Whereas the scattering pattern and model presented in the text (Figure 2b) correspond to 2-D sheets of hexagonally packed nanorods that have ordered into a 3-D HCP structure, the majority of SAXS patterns obtained from nanorod crystals consisted of only 2-D ordered sheets (Figure S5, S6). A modeled scattering pattern of a perfect 2-D hexagonal crystal shows excellent agreement with the experimental SAXS data obtained for most of the nanorod superlattices (Figure S21a). The peaks corresponding to 2-D hexagonal order also appear in the scattering pattern presented for the HCP structure (Figure S21b) with the peaks that are unaccounted for by the model for the 2-D lattice matching well with the additional peaks included in the modeled 3D structure (Figure $2 b$ ).
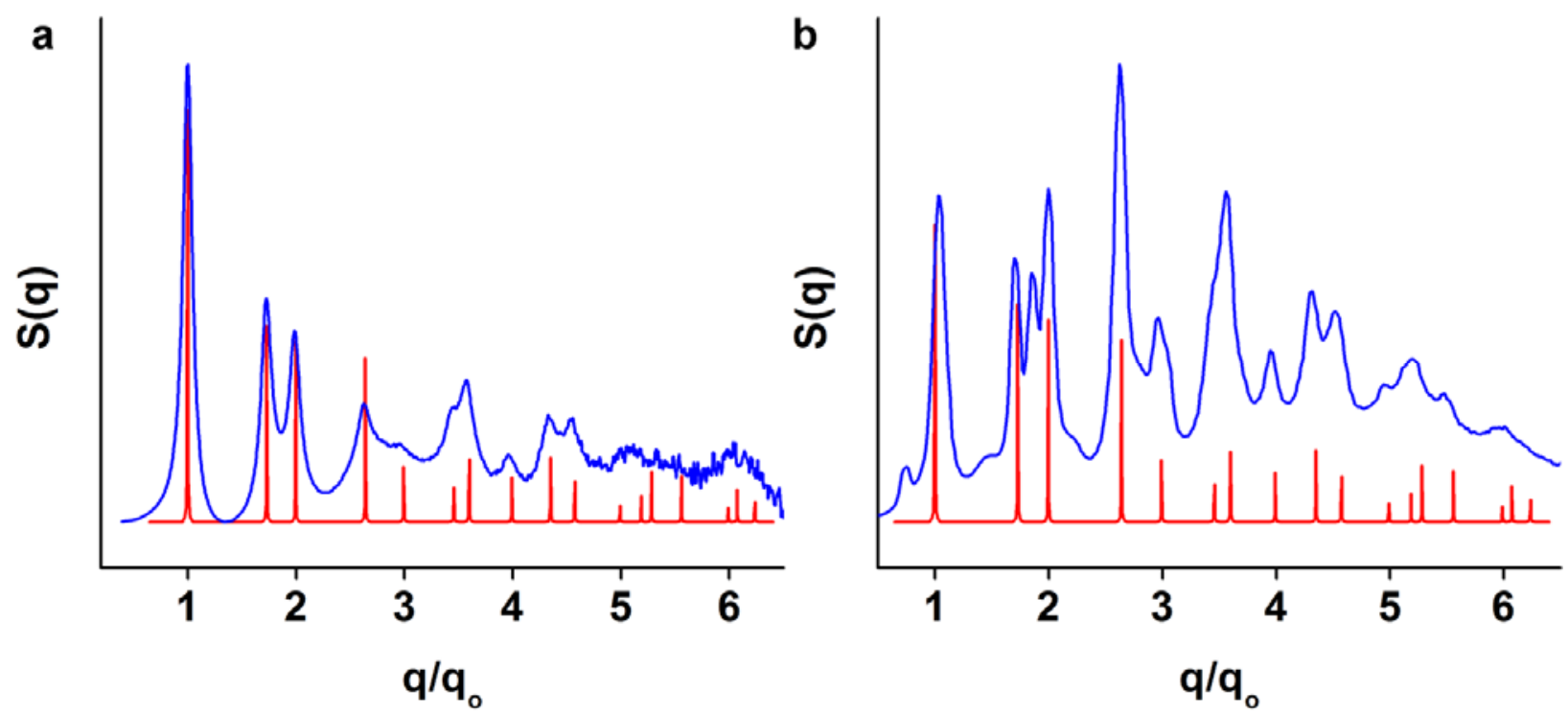

Figure S21. Observation of predominantly 2-D ordering in superlattices of gold nanorods. a, A scattering pattern representative of the majority of crystallized nanorod samples (blue) (see Figure S5) with the ideal scattering pattern from a perfect crystal with only 2-D hexagonal order (red). The agreement between the experimental and modeled peaks, in conjunction with the absence of any additional scattering peaks, suggests very little ordering between 2-D sheets of nanorods. b, A scattering pattern from a nanorod sample that underwent extensive thermal annealing, which ultimately resulted in 3-D ordering (blue trace, same as in Figure $2 \mathrm{~b}$ ), with the ideal scattering pattern from a perfect crystal with only 2-D hexagonal order (red). The scattering patterns match well but several peaks that are present in the experimental data are not present in the modeled pattern; these peaks are represented in the 3-D HCP model presented in Figure 2b. 


\section{Evidence for the Transition from 2-D to 3-D Order in Nanorod Superlattices}

In situ monitoring of a sample of nanorods during the annealing process demonstrated that it is the 2-D hexagonal layers of nanorods that crystallize first, and that 3-D ordering is only observed after long-range 2-D ordering is already present in the sample. This is noted by the fact that peaks corresponding to 2-D ordering of the rods are present in all scans, albeit with only short-range order at room temperature (Figure S22). As the temperature increases and more thermal energy is provided to the system, reorganization occurs and the domain size of the 2-D sheets increases (as indicated by the narrower, more well-defined peaks). It is only after significant annealing that peaks corresponding to 3-D ordering between 2-D sheets are observed (arrows, $57^{\circ} \mathrm{C}$ scans).

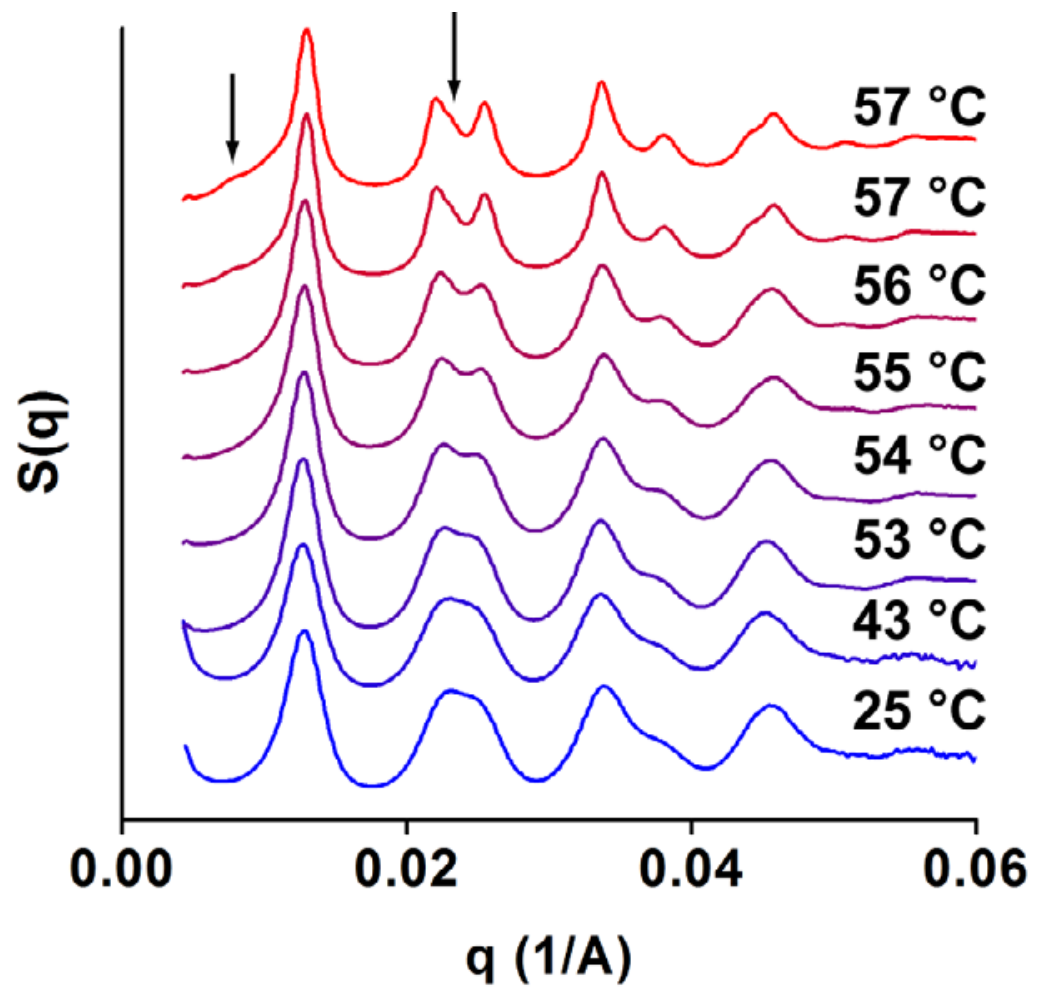

Figure S22. Analysis of the crystallization process of nanorods. Temperature-resolved SAXS scans of nanorods ( $55 \mathrm{~nm}$ length, $14 \mathrm{~nm}$ width) with the $\mathrm{n}=2$ linker, starting at low temperatures, then gradually increasing to just below the melting temperature of the sample $\left(\sim 57^{\circ} \mathrm{C}\right)$, where the sample was held for several minutes and scanned again. 


\section{The Possibility of Orientational Ordering Between 1-D Columns of Nanoprisms}

Although ordering between 2-D sheets of nanorods was observed, evidence for a similar, secondary assembly event for 1-D nanoprism superlattices was difficult to detect, even with significant thermal annealing. At elevated temperatures with extended annealing times, the presence of a weak, broad peak at low q values could be observed for some prism samples at a position consistent with hexagonal ordering between stacks of nanoprisms (Figure S23). However, the lack of additional, well-defined scattering peaks consistent with this formation makes a conclusive determination difficult, and points towards 3-D assemblies with at most only short range order.

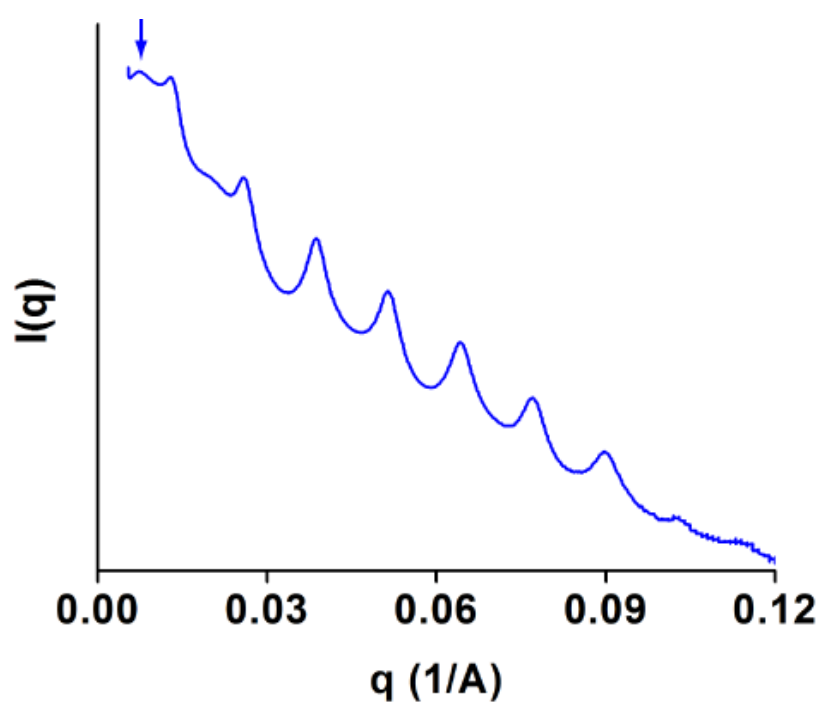

Figure S23. Evidence for weak association between 1D nanoprism superlattices. Scattering pattern for $60 \mathrm{~nm}$ edge length prisms crystallized with the $\mathrm{n}=1$ linker. The small peak indicated by the arrow is at the expected position for weak ordering between columns of prisms in a hexagonal arrangement. The nanoprism form factor has not been removed from these scans to ensure the small peak is not an artifact of the subtraction process.

A number of reasons exist for the inability of DNA-mediated hybridization to induce hierarchical 3-D assembly of nanoprisms: (1) DNA strands uniformly coat the surfaces of the nanoprisms ${ }^{1}$, indicating that the significantly smaller surface area along the edges of prisms contains fewer DNA strands that are able to participate in hybridization events relative to the large triangular faces. Nanoprisms with edge lengths of $140 \mathrm{~nm}, 95 \mathrm{~nm}$, and $60 \mathrm{~nm}$ have a ratio of triangular face surface area to edge face surface area of 5.4, 3.7, and 2.3, respectively. (2) DNA strands on the edges of prisms can more easily bend away from an orientation perpendicular to the nanoprism face due to decreased steric crowding. As we have previously shown $^{8}$, an increased flexibility in DNA linker strands results in lower localized concentrations of the DNA sticky ends, due to the fact that the DNA strands can adopt a larger number of configurations. This lower effective concentration of the DNA sticky ends along the edges of the prisms ultimately results in weaker interparticle interactions, making prism edge-edge 
association less stable than face-face association ${ }^{8}$. (3) After assembling into 1-D superlattices, distances between nanoprisms range from $\sim 25$ to $\sim 115 \mathrm{~nm}$. Since nanoprisms are $\sim 7 \mathrm{~nm}$ thick, the side of a column of assembled nanoprisms consists of small $\sim 7 \mathrm{~nm}$ patches of DNA spaced by considerable distances. This means that, even in 1-D stacks of prisms, the DNA density perpendicular to the prism edges (along the length of a stack), is significantly lower than what is observed perpendicular to the faces of the prisms. It is possible for stacks to make weak associations with each other without registry of the prisms, which results in the relatively disordered 3-D assemblies of prism superlattices observed in Figure S23. However, a perfectly ordered 3-D lattice is unlikely to occur, as it is not significantly favorable over other, more disordered states.

While entropic effects have been shown to induce ordering between plate and rod-like objects as in nematic and discotic columnar liquid crystalline phases, this type of assembly requires nanoparticle concentrations significantly higher than what has been used in this work (crystallized nanoprisms are at most $200 \mathrm{nM}$ ). For example, Lekkerkerker and coworkers found nematic phases of plate-like nanostructures at concentrations above $\sim 30 \mu \mathrm{M}^{14}$, and Zhang et al. showed that ordering between columnar stacks of triangular nanoprisms occurs at concentrations of $\sim 50 \mu \mathrm{M}^{15}$. Therefore, although entropic effects may direct the formation of liquid crystalline phases of 1-D columns of nanoprisms at high concentrations, this study focuses on relatively dilute concentration regimes wherein DNA-mediated interactions are more relevant. 
Origin of Oscillations in the Intensity of Diffraction Peaks from Assembled Triangular Nanoprisms

After form factor correction, nearly all columnar superlattices of triangular nanoprisms exhibit a distinct oscillation in the intensity of the scattering pattern (Figure 2d, S7, S8, S9). The oscillation in peak intensities can be attributed to the uniform thickness of the prism; this effect is commonly observed in SAXS investigations of lipid bilayers ${ }^{16}$. Importantly, this oscillation does not affect the positions of the scattering peaks which are used to determine lattice spacing and domain size. 


\section{Evidence for the Orientational Alignment of Rhombic Dodecahedra in FCC Superlattices}

In order to probe the orientational alignment of rhombic dodecahedra within a superlattice, several probable cases were modeled and compared to the experimental scattering plot. The modeled data in which face-to-face association is maximized shows the best correlation to the experimental data (Figure S24, S25).

Rotation around $[0,0,1]$ with an angle $=0$
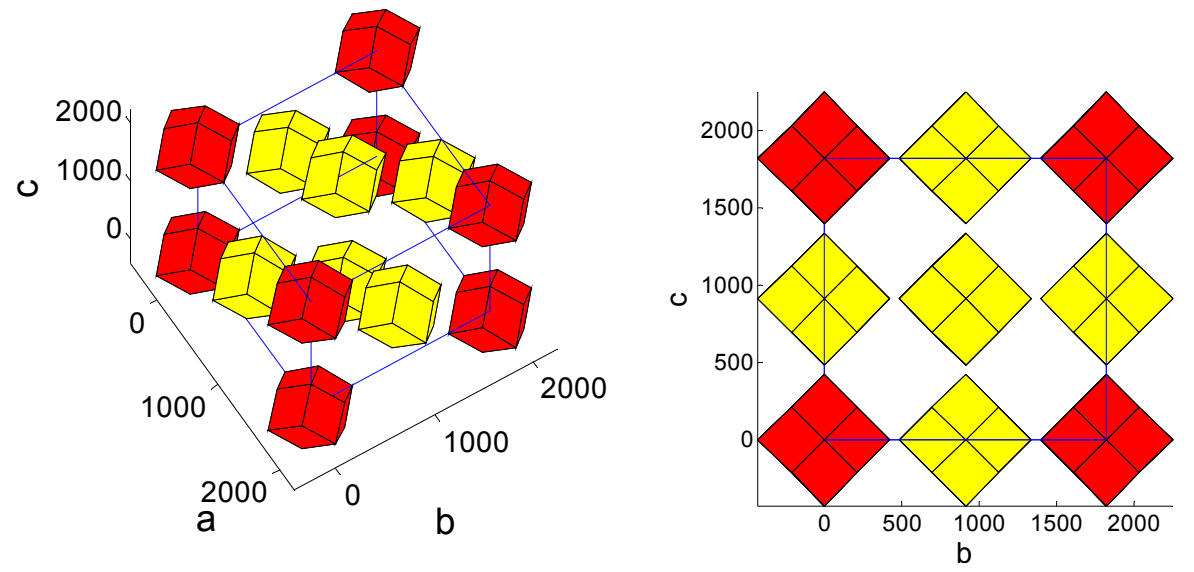

Figure S24. Schematic illustration of rhombic dodecahedra in an FCC lattice with a rotation angle about $[0,0,1]$ of 0 . All rhombic dodecahedra are equivalent in this lattice, but are displayed with two different colors to aid in visualization of the $3 \mathrm{D}$ unit cell.

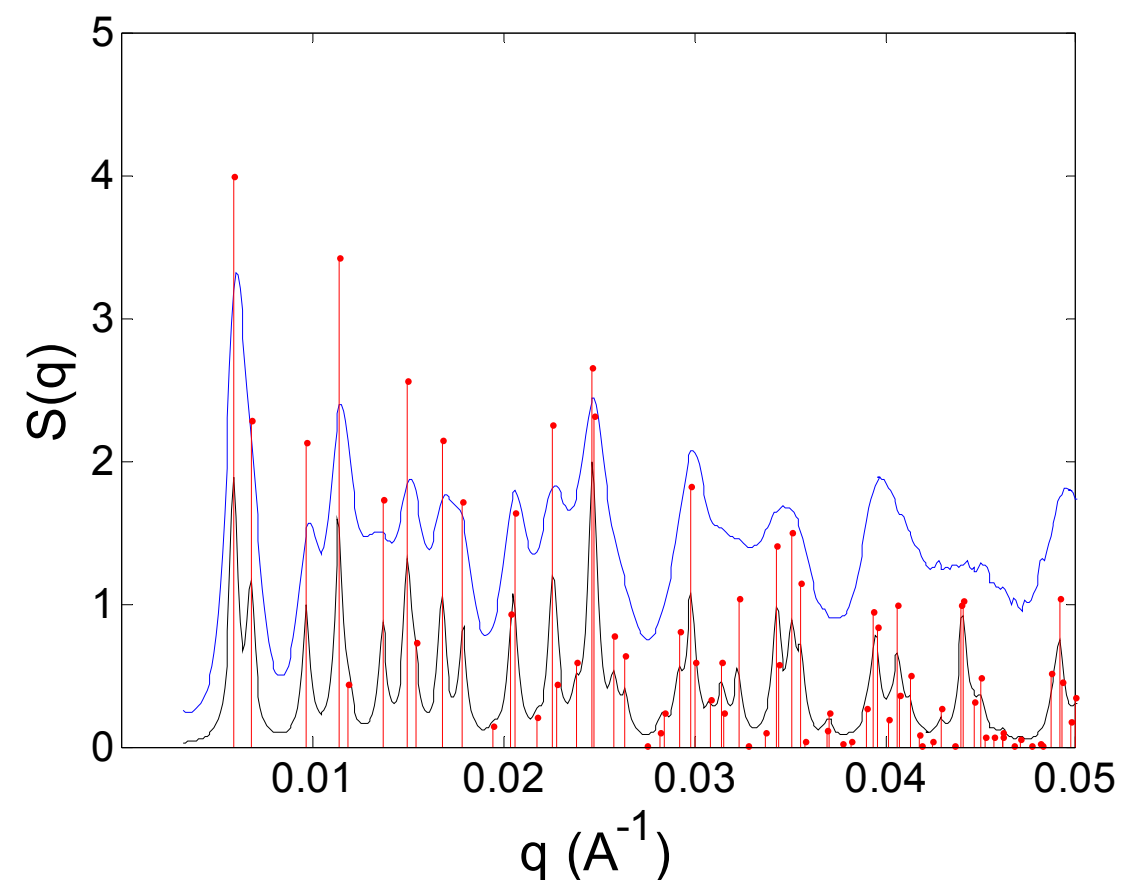

Figure S25. Comparison of experimental and simulated scattering patterns to investigate rhombic dodecahedra orientational alignment. Experimental SAXS data for rhombic dodecahedra (blue) with simulated peaks for rhombic dodecahedra in an FCC arrangement with a rotation angle about $[0,0,1]$ of 0 (black and red). The excellent agreement between the experiment and the simulation suggest rhombic dodecahedra adopt a preferential face-to-face orientation within the FCC unit cell. 
Rotation around $[0,0,1]$ with an angle $=\pi / 4$
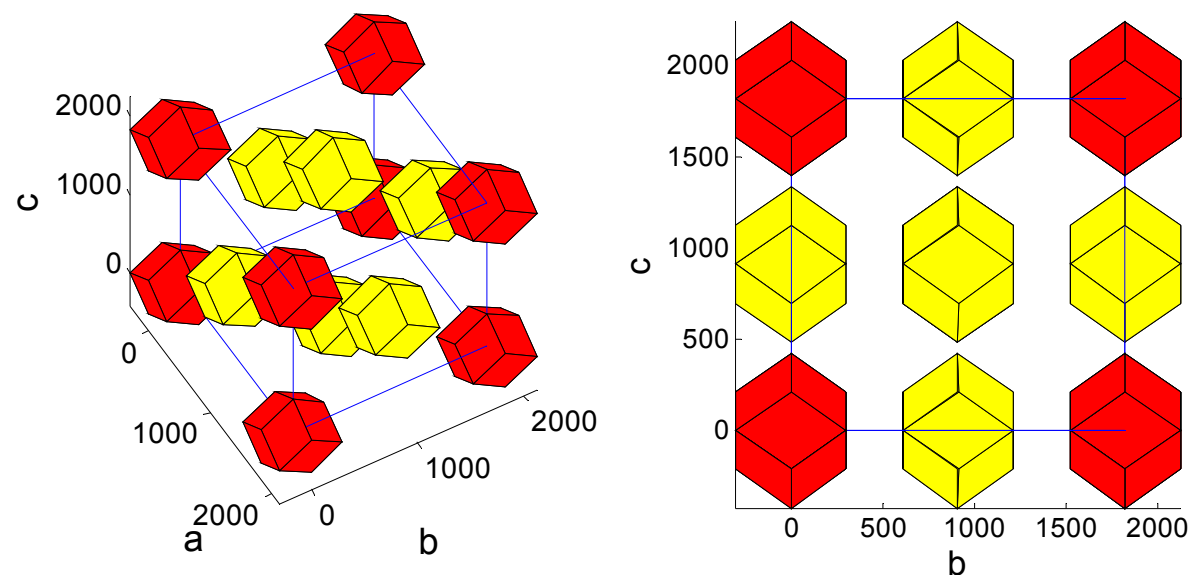

Figure S26. Schematic illustration of rhombic dodecahedra in an FCC lattice with a rotation angle about $[0,0,1]$ of $\pi / 4$. All rhombic dodecahedra are equivalent in this lattice, but are displayed with two different colors to aid in visualization of the 3D unit cell.

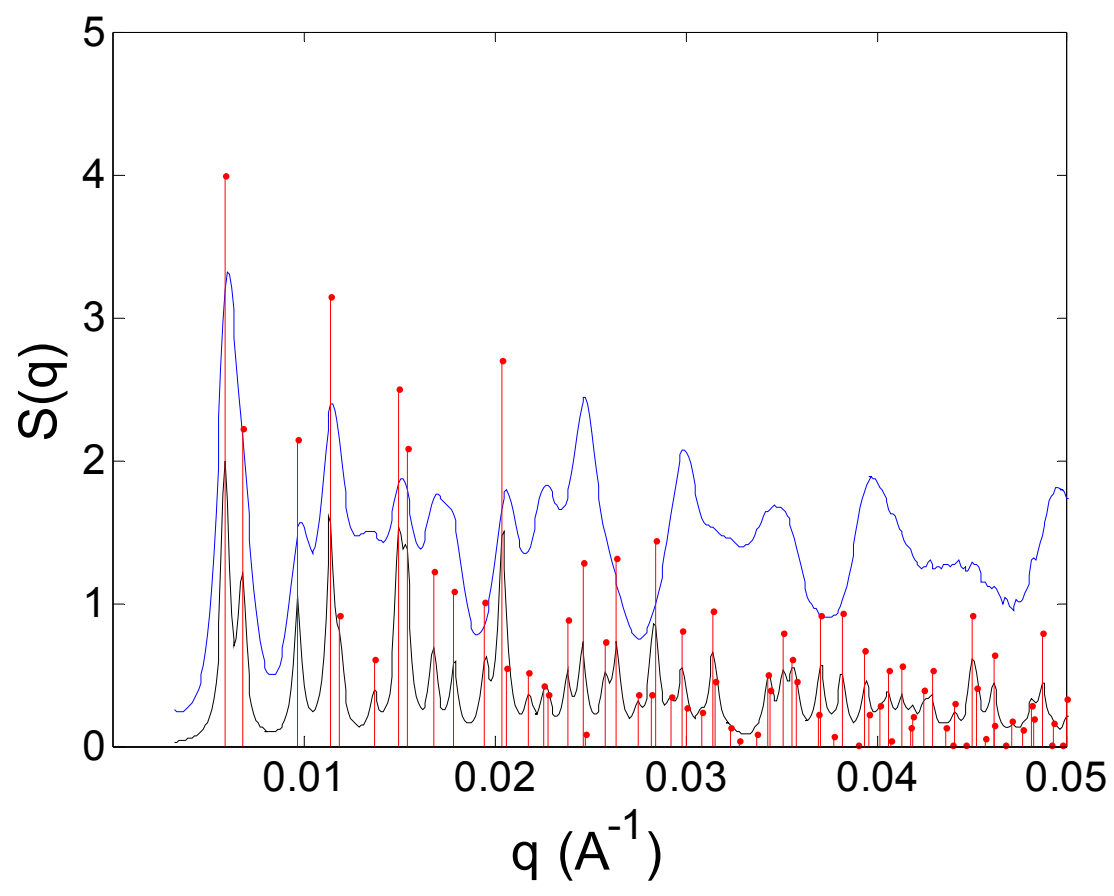

Figure S27. Comparison of experimental and simulated scattering patterns to investigate rhombic dodecahedra orientational alignment. Experimental SAXS data for rhombic dodecahedra (blue) with simulated peaks for rhombic dodecahedra in an FCC arrangement with a rotation angle about $[0,0,1]$ of $\pi / 4$ (black and red). The lack of agreement between the experiment and the simulation (most notable at q values $>0.02$ ) suggest rhombic dodecahedra do not adopt this orientation within the FCC unit cell. 
Rotation around $[0,0,1]$ with an angle $=\pi / 3$
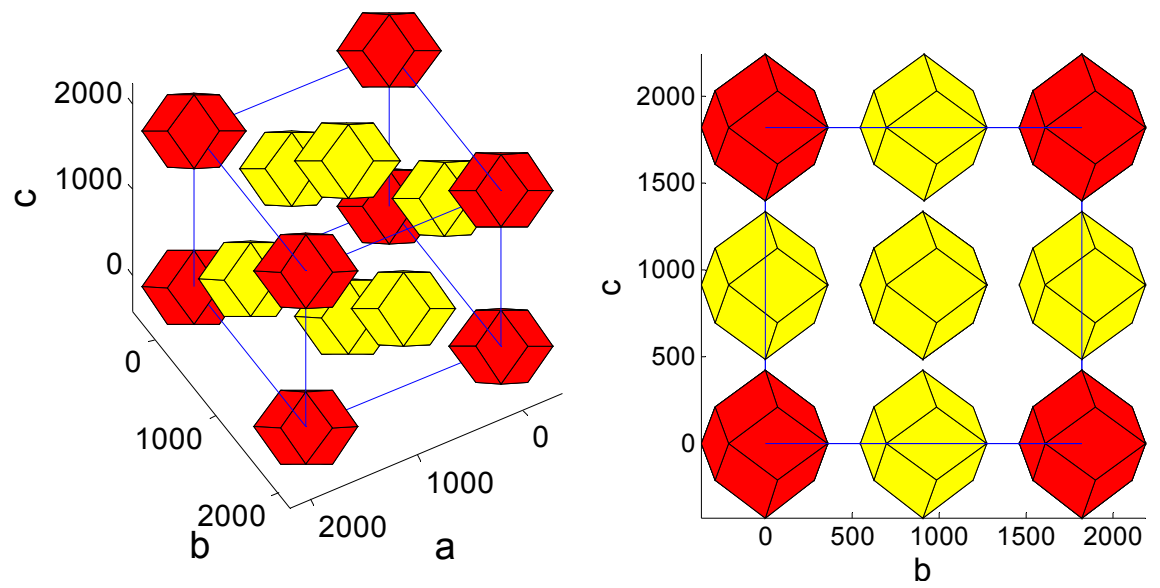

Figure S28. Schematic illustration of rhombic dodecahedra in an FCC lattice with a rotation angle about $[0,0,1]$ of $\pi / 3$. All rhombic dodecahedra are equivalent in this lattice, but are displayed with two different colors to aid in visualization of the $3 \mathrm{D}$ unit cell.

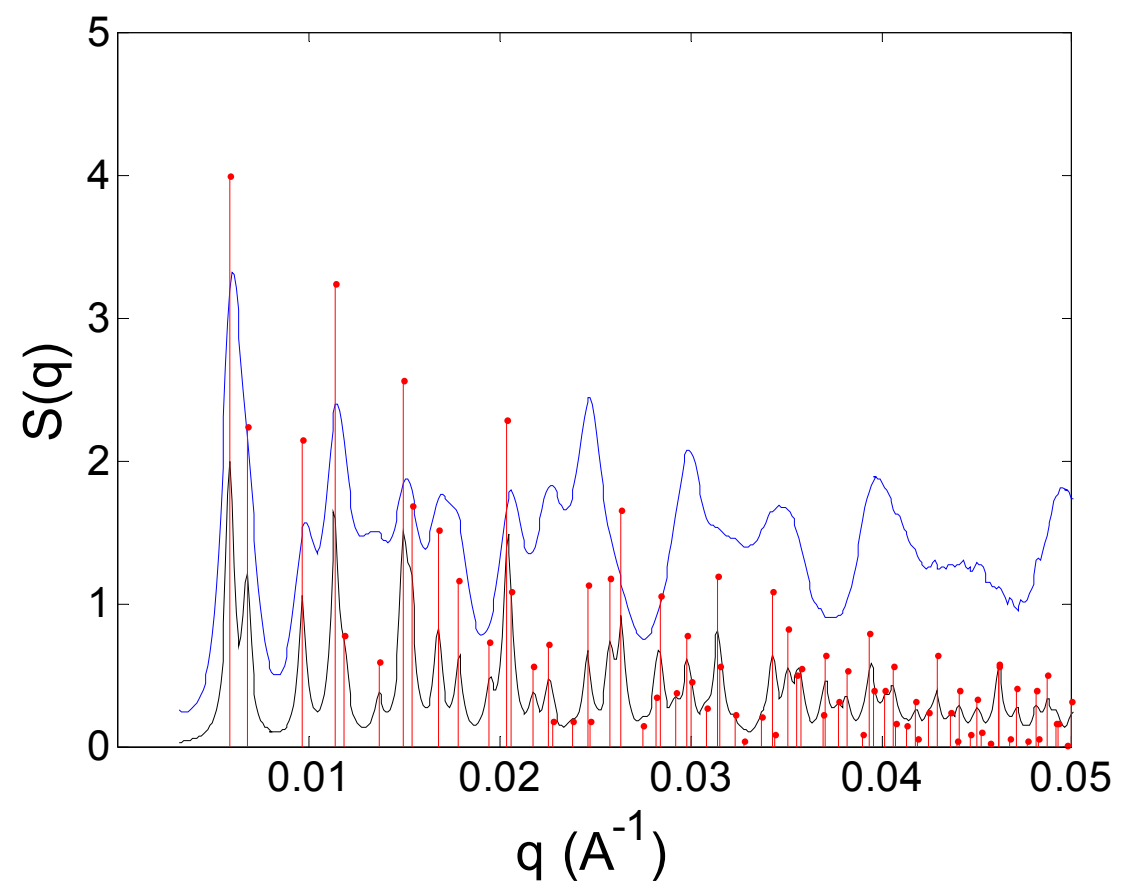

Figure S29. Comparison of experimental and simulated scattering patterns to investigate rhombic dodecahedra orientational alignment. Experimental SAXS data for rhombic dodecahedra (blue) with simulated peaks for rhombic dodecahedra in an FCC arrangement with a rotation angle about $[0,0,1]$ of $\pi / 3$ (black and red). The lack of agreement between the experiment and the simulation suggest rhombic dodecahedra do not adopt this orientation within the FCC unit cell. 


\section{Analysis and Discussion of the Assembly of Octahedra with Short and Intermediate DNA Lengths}

As has been previously stated ${ }^{8,17}$, the driving force for DNA-mediated nanoparticle crystallization is the maximization of DNA hybridization interactions between particles - this can be correlated with the maximum amount of face-to-face overlap between neighboring nanostructures. When considering octahedra, it is important to note that it is not possible to align the particles in an ordered lattice with completely commensurate face-to-face interactions. Therefore, in order to determine the 3D structure of octahedra assembled with short DNA strands (Figure 3b), different possible lattices were examined.

First, the theoretical densest packing of octahedra ${ }^{18}$ is presented as follows:

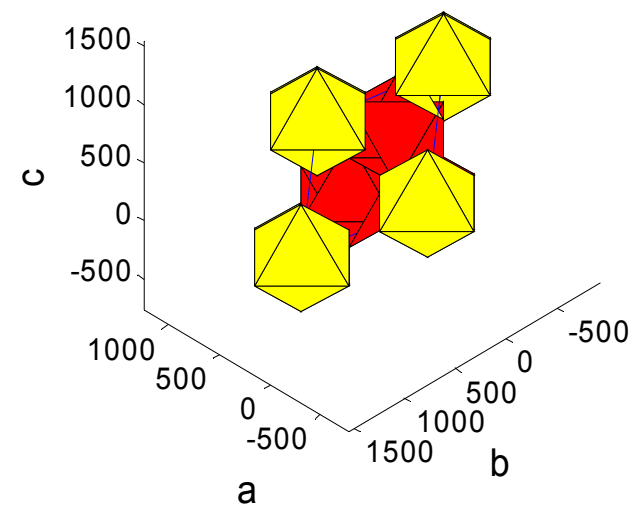

Figure S30. Schematic illustration of the densest packing of octahedra. All octahedra are equivalent, however, different colors are used to aid visualization of the 3D lattice.

This structure has two types of face-to-face interactions:

Type A

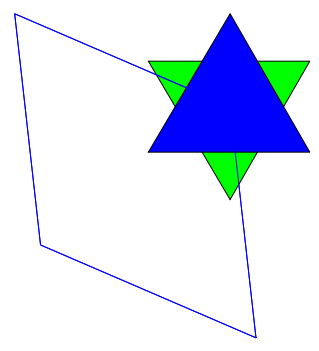

Type B

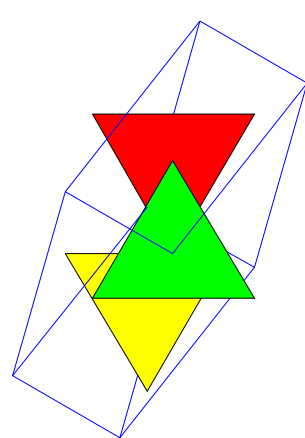

Figure S31. Schematic illustration of two types of face-to-face interactions present in the densest packing of octahedra structure with an interparticle distance of $\mathbf{0 ~} \mathbf{~ m}$. Different colors are used to aid in visualization of the face-to-face interactions. Interactions of type A exhibit much greater face-to-face overlap between nearest neighbors, which results in a greater number of favorable hybridization interactions between DNA linking strands. 
2 of 8 faces of the octahedra exhibit type A interactions with their nearest neighbors, while 6 of 8 faces exhibit type B interactions.

When assembling octahedra with DNA linkers, the face-to-face distance between particles is uniformly increased by the length of the DNA strands. In this case, type B interactions exhibit reduced face-to-face overlap with increasing lattice parameters, due to the geometry of the lattice ${ }^{18}$ (Figure S32). (The amount of face-to-face overlap of type A does not change with increasing particle distance.) This indicates that, when assembling octahedra with DNA, the theoretical densest packing of octahedra is not an optimal structure, as it has minimal face-to-face overlap between neighboring particles and therefore minimal DNA hybridization interactions.
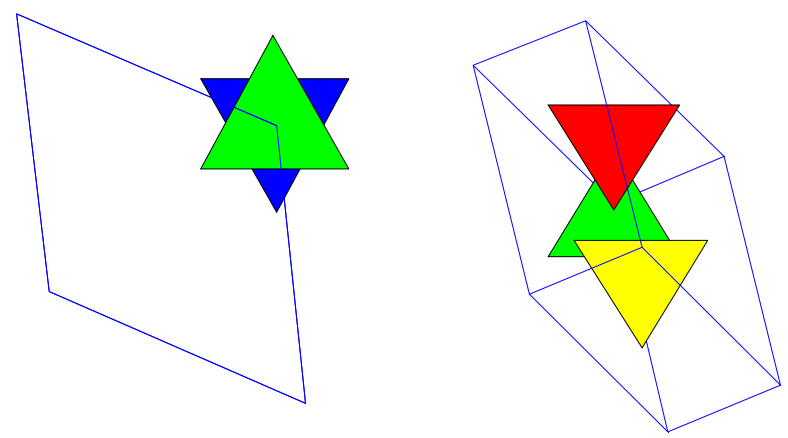

Figure S32. Schematic illustration of two types of face-to-face interactions present in the densest packing of octahedra structure with particles separated by 15 nm DNA linkers. Different colors are used to aid in visualization of the face-to-face interactions.

Other experimentally obtained lattices ${ }^{19-20}$ exhibit the same problem-minimal face-to-face overlap is achieved when the particles are aligned in these structures. Importantly, this problem is exacerbated when the lattice parameters increase.

Based on previous calculations ${ }^{8-9}$ and comparisons with the DNA lengths for the assemblies studied in this work, we can estimate that the DNA length for the shortest interparticle distance $(n=0)$ is approximately $15 \mathrm{~nm}$. If we calculate the scattering pattern for octahedra assembled with $15 \mathrm{~nm}$ DNA linkers into the lattice described above, the lattice parameters should be $122 \mathrm{~nm}$ and $68.5 \mathrm{~nm}$ for a and c, respectively (Figure S33, S34). 


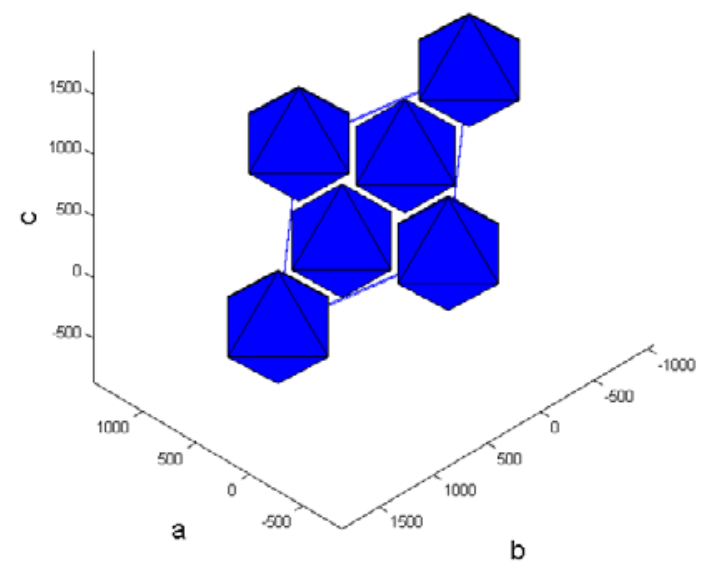

Figure S33. Schematic illustration of the densest packing of octahedra for short (15 nm) DNA lengths.

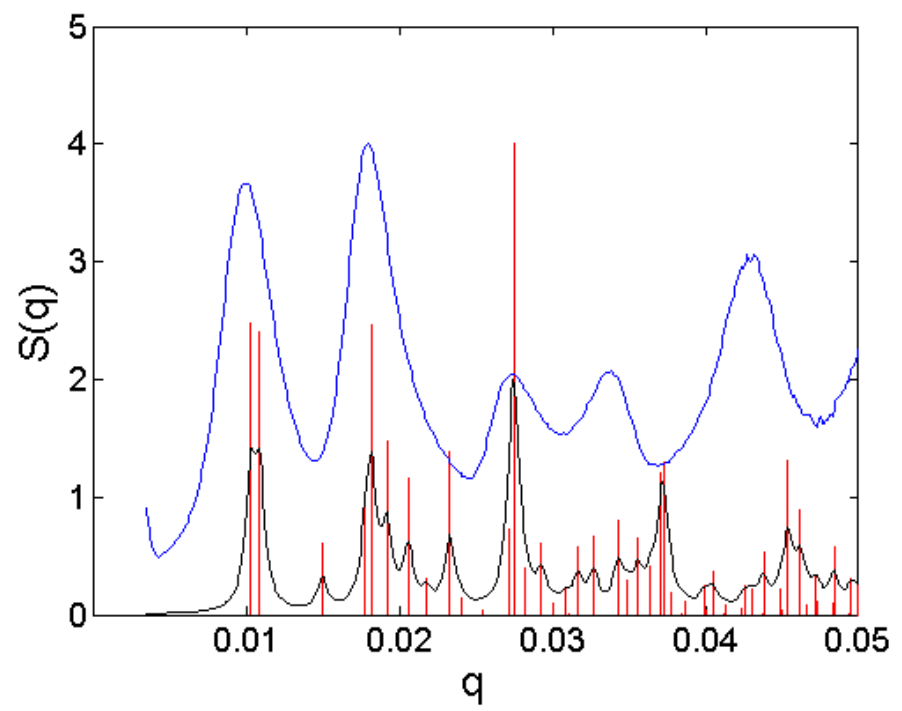

Figure S34. Evidence of a disordered arrangement of octahedra with short DNA lengths. Experimental scattering pattern from octahedra with short DNA length (blue) with simulated scattering pattern from octahedra in a densest packed arrangement (black and red) with 15 face-to-face distance (Figure S33). The lack of agreement between the experiment and the simulation at large $\mathrm{q}$ values suggest octahedra do not adopt a dense packed arrangement for short DNA lengths.

While the simulation does present a limited degree of matching with the experimental data, there is little correlation for peak intensities at $q$ values $>0.025$ and in peak positions at $q$ values $>0.03$. Additionally, the breadth of the peaks indicates that, despite a small amount of correspondence with the predicted scattering pattern, there is no long range order (which would 
manifest as sharp diffraction peaks, as can be seen in most other scattering patterns presented in this work).

Secondly, we considered the possibility of octahedra packing into a BCC lattice with short DNA. This presented a logical choice, as longer DNA linkers crystallized octahedra into BCC lattices (Figure 3c, S12). We again assume that the face-to-face distance for nearest neighbor particles is $15 \mathrm{~nm}$. In this case, the lattice parameter should be $79.2 \mathrm{~nm}$, and the amount of face-to-face overlap should be increased relative to the theoretically determined densest packing (vide supra), as each particle should have 8 interactions of type A instead of 2 . However, the particle and lattice geometries prevent this from being a stable structure, as this lattice parameter would result in overlap between the particle tips (Figure S35).

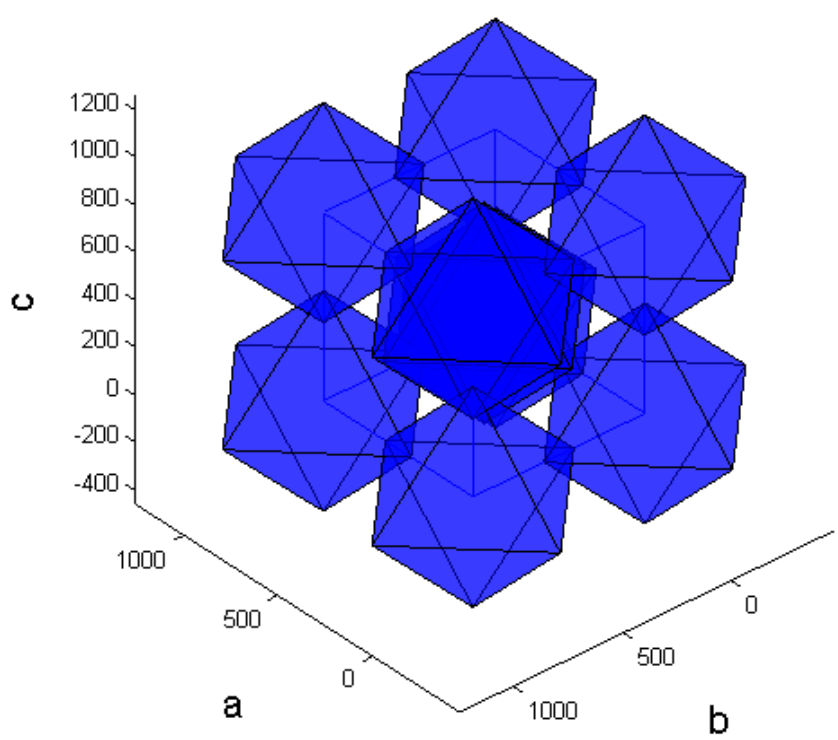

Figure S35. Schematic illustration of the BCC packing of octahedra for short (15 nm) DNA lengths showing overlap between particles.

This indicates that this lattice is not possible unless the particle tips are significantly truncated. However, even assuming that the particle tips are truncated enough to allow such a structure, the modeled scattering pattern does not provide a good match to the experimental data (Figure S36). 


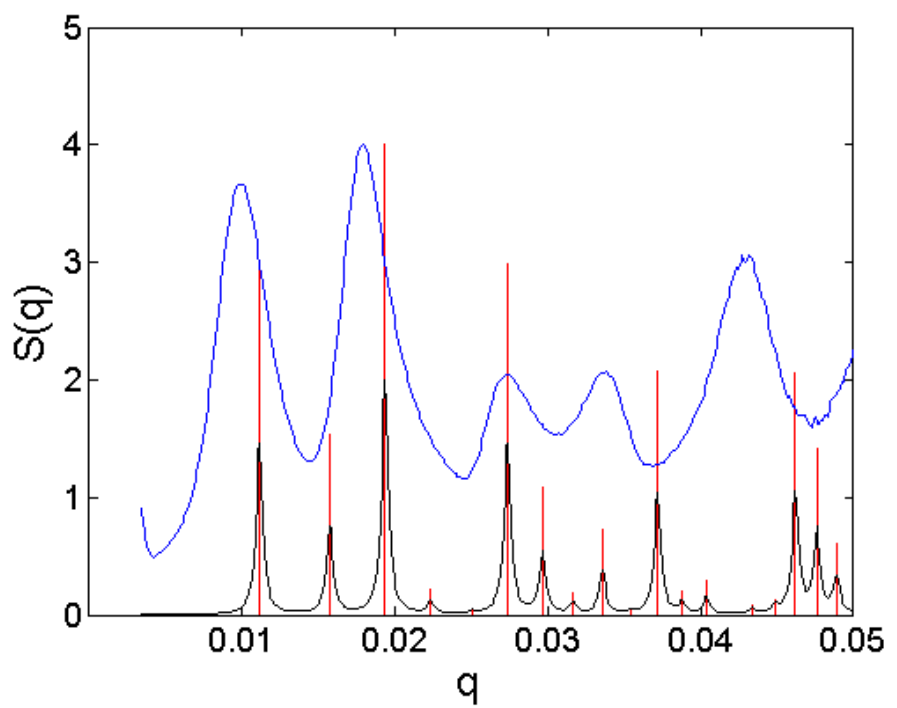

Figure S36. Evidence of a disordered arrangement of octahedra with short DNA lengths. Experimental scattering pattern from octahedra with short DNA length (blue) with simulated scattering pattern from octahedra in a BCC arrangement (black and red) with 15 face-to-face distance (Figure S35). The lack of agreement between the experiment and the simulation suggest octahedra do not adopt a BCC arrangement for short DNA lengths.

Of the lattices presented above, the experimental data of octahedra assembled with short DNA match more closely to the densest packed structure than to the BCC packing. However, thermodynamically we would predict that the BCC lattice should be the preferred structure considering its larger degree of face-to-face interactions. The interplay between these and other possible structures, combined with the relative rigidity of short DNA results in frustration of the assembled structure ${ }^{8}$, which in turn causes only short range order as demonstrated in the pair distribution function below:

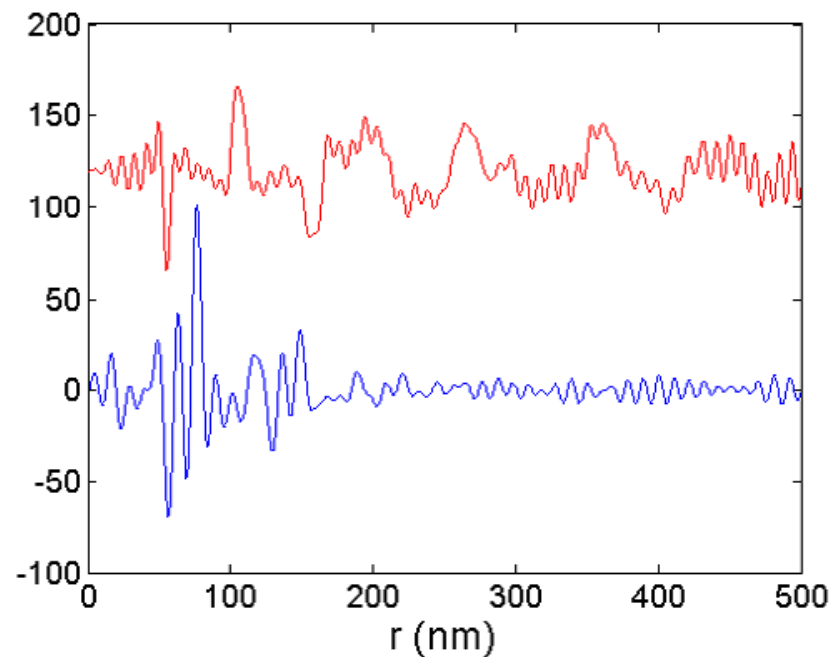

Figure S37. Pair distribution function analysis of octahedra superlattice scattering patterns. Pair distribution functions for assembled octahedra with short (blue, $n=0$ ) and intermediate (red, $n=1$ ) DNA lengths. 
From these curves, the nearest center-to-center distances for the two samples are obtained: $76.8 \mathrm{~nm}$ for the $\mathrm{n}=0$ linker (blue) and $105 \mathrm{~nm}$ for the $\mathrm{n}=1$ linker (red, presented as a comparison). The distance between the center of an octahedron and the center of one of its faces is $23.9 \mathrm{~nm}$. This means that, if the particles were aligned in a commensurate face-to-face arrangement, the interparticle distance for the $\mathrm{n}=0$ linker would be predicted to be $\sim 63 \mathrm{~nm}-$ much lower than the interparticle distance calculated via the PDF. This indicates that the lattice does not exhibit significant ordering through commensurate face-to-face interactions.

Additionally, the octahedra superlattice with short, $n=0$ DNA (blue curve) has much shorter range ordering than that with intermediate DNA (red curves) as seen from the fast damping of correlation peaks. 


\section{Evidence for the Orientational Alignment of Octahedra in BCC Superlattices}

In order to probe the orientational alignment of octahedra within a BCC superlattice, several probable orientations were modeled and compared to the experimental scattering data. The case in which face-to-face association is maximized shows the best correlation to the experimental data (Figure S38, S39).

Rotation around $[0,0,1]$ with an angle $=\pi / 4$
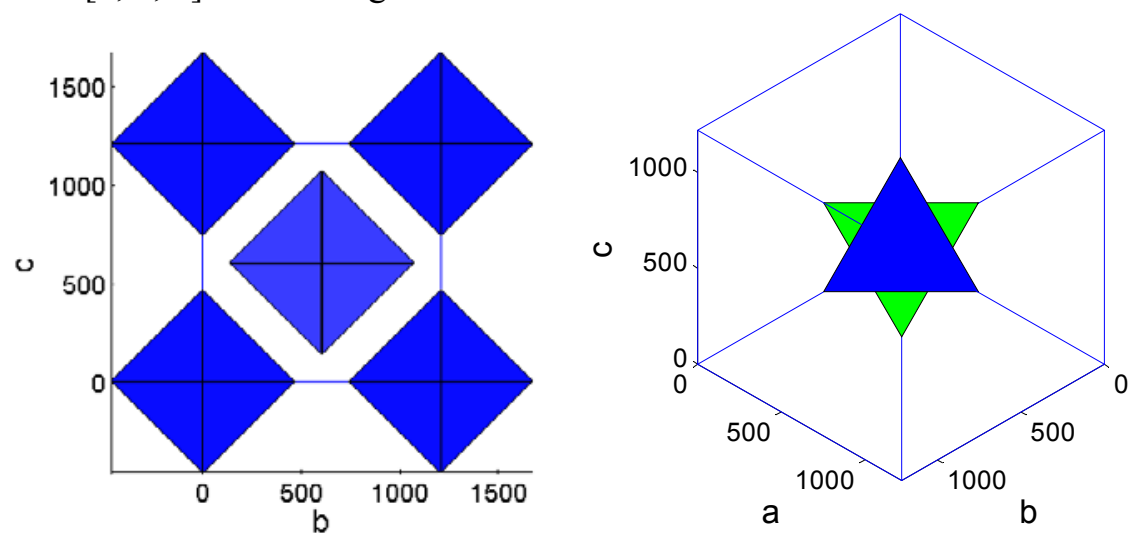

Figure S38. Evidence of a BCC arrangement of octahedra. Schematic illustration of octahedra in a BCC lattice with a rotation angle about $[0,0,1]$ of $\pi / 4$ (left). Illustration of the face-to-face overlap between neighboring triangular faces of octahedra in this arrangement (right).

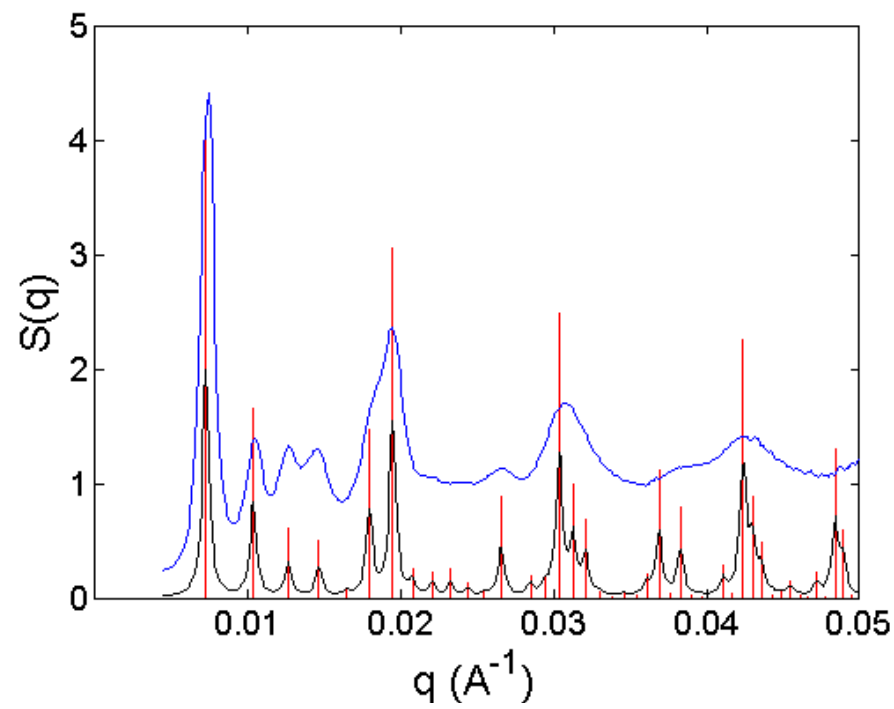

Figure S39. Comparison of experimental and simulated scattering patterns to investigate octahedra orientational alignment. Experimental SAXS data for octahedra (blue) with simulated peaks for octahedra in a BCC arrangement with a rotation angle about $[0,0,1]$ of $\pi / 4$ (black and red). The excellent agreement between the experiment and the simulation suggest octahedra adopt a preferential face-to-face orientation within the BCC unit cell 
Rotation around $[0,0,1]$ with an angle $=0$

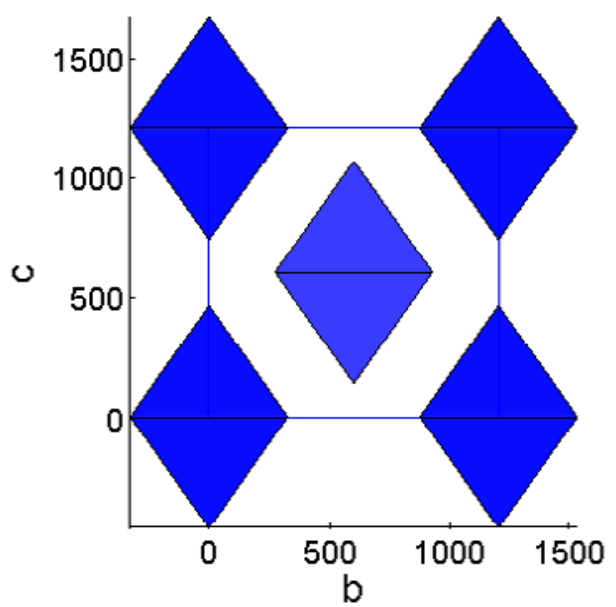

Figure S40. Schematic illustration of octahedra in a BCC lattice with a rotation angle about $[0,0,1]$ of 0 .

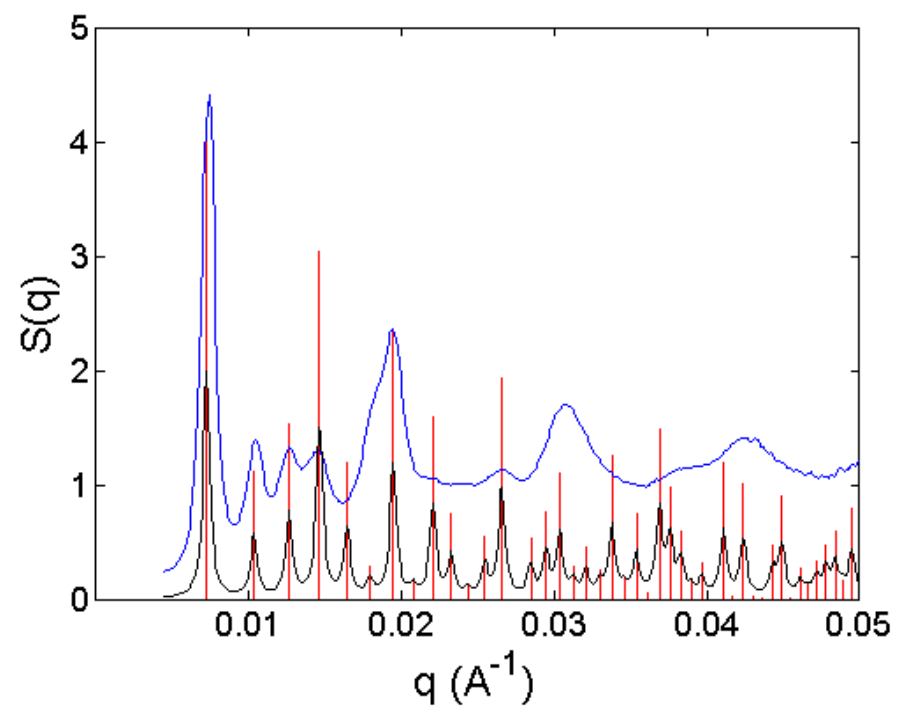

Figure S41. Comparison of experimental and simulated scattering patterns to investigate octahedra orientational alignment. Experimental SAXS data for octahedra (blue) with simulated peaks for octahedra in a BCC arrangement with a rotation angle about $[0,0,1]$ of 0 (black and red). The poor correlation between the experiment and the simulation relative to the $\pi / 4$ rotation angle case suggests octahedra do not adopt this orientation within the BCC unit cell. 
Rotation around $[0,0,1]$ with an angle $=\pi / 3$

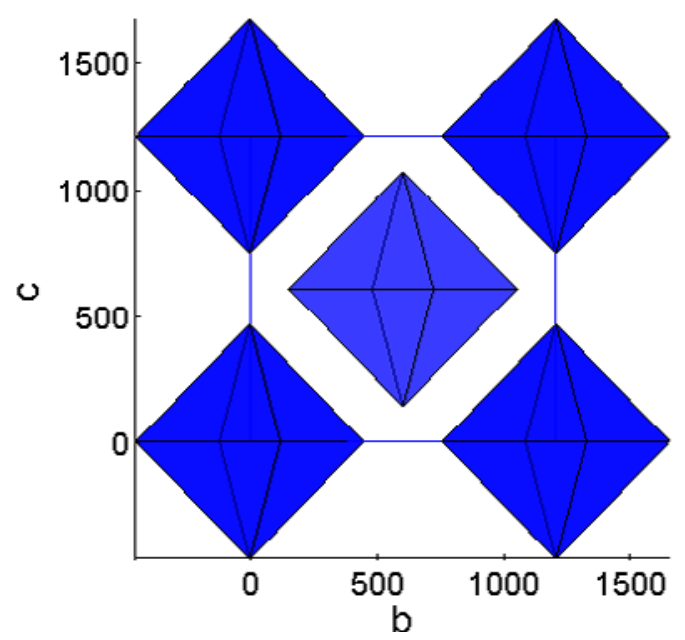

Figure S42. Schematic illustration of octahedra in a BCC lattice with a rotation angle about $[0,0,1]$ of $\pi / 3$.

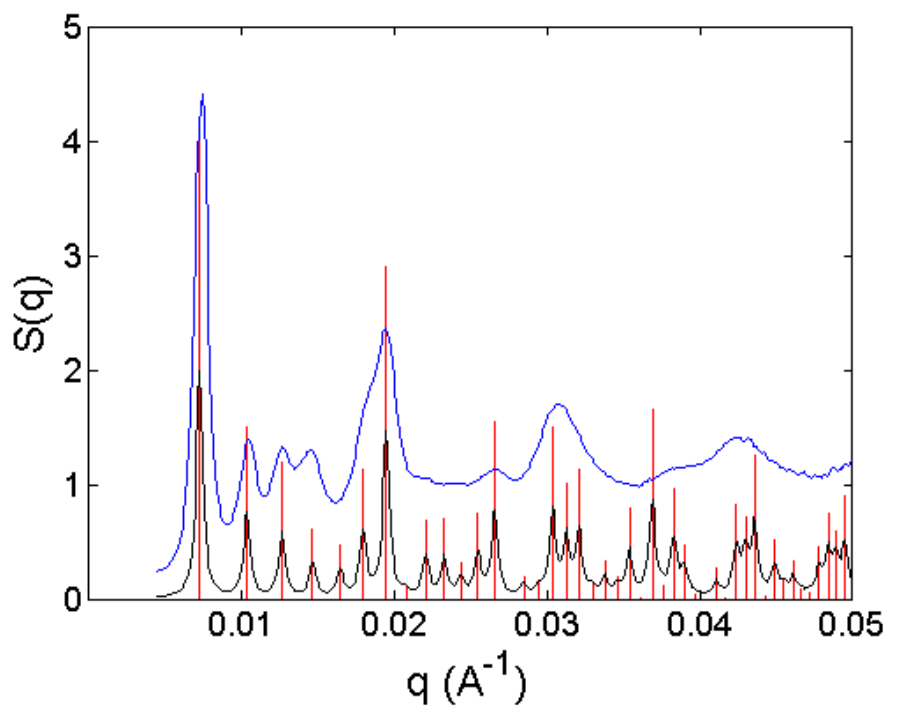

Figure S43. Comparison of experimental and simulated scattering patterns to investigate octahedra orientational alignment. Experimental SAXS data for octahedra (blue) with simulated peaks for octahedra in a BCC arrangement with a rotation angle about $[0,0,1]$ of $\pi / 3$ (black and red). The poor correlation between the experiment and the simulation relative to the $\pi / 4$ rotation angle case suggests octahedra do not adopt this orientation within the BCC unit cell. 


\section{Evidence for a BCC to FCC Phase Transformation in Octahedra Superlattices}

In addition to observing a BCC lattice of octahedra for intermediate DNA lengths ( $\mathrm{n}=1$, $2)$ and an FCC lattice of octahedra for long DNA $(n=4)$, we also observed a scattering pattern which indicates both BCC and FCC lattices are present for a DNA length in-between these two cases $(n=3)$. In this scattering plot, peaks are observed that index to both BCC and FCC ordering (Figure S44). We hypothesize that at this DNA length there are domains of each type of lattice within the sample, which provides further evidence of a nanoparticle superlattice phase transformation as a function of DNA length and nanostructure shape.

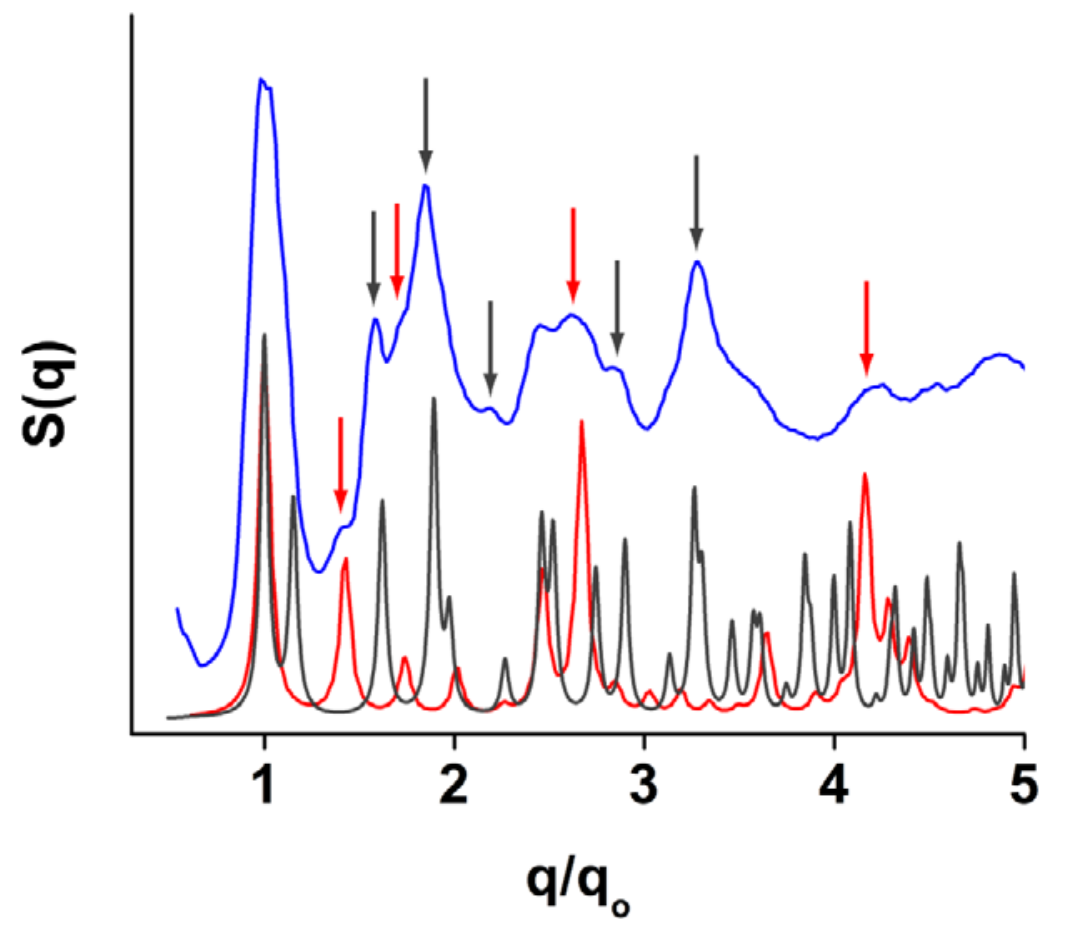

Figure S44. Evidence of a phase transformation in assembled octahedra. Whereas octahedra crystallized with short DNA lengths $(n=1,2)$ showed BCC ordering and octahedra crystallized with long DNA $(n=4)$ showed FCC ordering (Figure 3c,d; Figure S12), an intermediate DNA length $(\mathrm{n}=3)$ demonstrated both BCC and FCC ordering. Experimental data (blue) exhibit peaks corresponding to both a BCC arrangement of octahedra with orientational order (red) and an FCC arrangement of octahedra without orientational order (grey). Arrows denote specific peaks which are unique to BCC (red) and FCC (grey) lattices. 


\section{References}

1 Millstone, J. E. et al. DNA-Gold Triangular Nanoprism Conjugates. Small 4, 2176-2180 (2008).

2 Hill, H. D. \& Mirkin, C. A. The bio-barcode assay for the detection of protein and nucleic acid targets using DTT-induced ligand exchange. Nat. Protoc. 1, 324-336 (2006).

3 Nikoobakht, B. \& El-Sayed, M. A. Preparation and Growth Mechanism of Gold Nanorods (NRs) Using Seed-Mediated Growth Method. Chem. Mater. 15, 1957-1962 (2003).

4 Jin, R., Wu, G., Li, Z., Mirkin, C. A. \& Schatz, G. C. What Controls the Melting Properties of DNA-Linked Gold Nanoparticle Assemblies? J. Am. Chem. Soc. 125, 16431654 (2003).

5 Liu \& Guyot-Sionnest, P. Mechanism of Silver(I)-Assisted Growth of Gold Nanorods and Bipyramids. J. Phys. Chem. B. 109, 22192-22200 (2005).

6 Millstone, J. E., Wei, W., Jones, M. R., Yoo, H. \& Mirkin, C. A. Iodide Ions Control Seed-Mediated Growth of Anisotropic Gold Nanoparticles. Nano Lett. 8, 2526-2529 (2008).

7 Niu, W. et al. Selective Synthesis of Single-Crystalline Rhombic Dodecahedral, Octahedral, and Cubic Gold Nanocrystals. J. Am. Chem. Soc. 131, 697-703 (2008).

8 Macfarlane, R. J. et al. Establishing the Design Rules for DNA-Mediated Programmable Colloidal Crystallization. Angew. Chem. Int. Edit. 49, 4589-4592 (2010).

9 Hill, H. D. et al. Controlling the Lattice Parameters of Gold Nanoparticle FCC Crystals with Duplex DNA Linkers. Nano Lett. 8, 2341-2344 (2008).

10 Hurst, S. J., Lytton-Jean, A. K. R. \& Mirkin, C. A. Maximizing DNA Loading on a Range of Gold Nanoparticle Sizes. Anal. Chem. 78, 8313-8318 (2006).

11 Cheng, W. et al. Probing in Real Time the Soft Crystallization of DNA-Capped Nanoparticles. Angew. Chem. Int. Edit. 49, 380-384 (2010).

12 Smith, B. D. \& Liu, J. Assembly of DNA-Functionalized Nanoparticles in Alcoholic Solvents Reveals Opposite Thermodynamic and Kinetic Trends for DNA Hybridization. J. Am. Chem. Soc. 132, 6300-6301 (2010).

13 Renaud, G., Lazzari, R. \& Leroy, F. Probing surface and interface morphology with Grazing Incidence Small Angle X-Ray Scattering. Surf. Sci. Rep. 64, 255-380 (2009).

14 van der Kooij, F. M., van der Beek, D. \& Lekkerkerker, H. N. W. Isotropic-Nematic Phase Separation in Suspensions of Polydisperse Colloidal Platelets. J. Phys. Chem. B. 105, 1696-1700 (2001).

15 Zhang, Z., Horsch, M. A., Lamm, M. H. \& Glotzer, S. C. Tethered Nano Building Blocks: Toward a Conceptual Framework for Nanoparticle Self-Assembly. Nano Lett. 3, 1341-1346 (2003).

16 Tristram-Nagle, S. \& Nagle, J. F. Lipid bilayers: thermodynamics, structure, fluctuations, and interactions. Chem. Phys. Lipids 127, 3-14 (2004).

17 Park, S. Y. et al. DNA-programmable nanoparticle crystallization. Nature 451, 553-556 (2008).

18 Torquato, S. \& Jiao, Y. Dense packings of the Platonic and Archimedean solids. Nature 460, 876-879 (2009).

19 Lu, W. et al. Super Crystal Structures of Octahedral c-In2O3 Nanocrystals. J. Am. Chem. Soc. 130, 6983-6991 (2008). 
20 Xie, S. et al. Supercrystals from Crystallization of Octahedral MnO Nanocrystals. $J$. Phys. Chem. C. 113, 19107-19111 (2009). 